\title{
THE ANNUAL MEETING IN BALTIMORE
}

The Sixtieth Annual Meeting of the American Mathematical Society was held at the Johns Hopkins University on Monday through Wednesday, December 28-30, 1953 in conjunction with the annual meeting of the Mathematical Association of America, which extended through Thursday, December 31.

About 600 registered for the meeting, including the following 500 members of the Society:

J. C. Abbott, Milton Abramowitz, J. W. Addison, Jr., M. I. Aissen, R. W. Allen, C. B. Allendoerfer, S. A. Amitsur, R. D. Anderson, M. M. Andrew, N. C. Ankeny, H. A. Antosiewicz, Hirotada Anzai, Richard Arens, K. J. Arnold, L. A. Aroian, Sholom Arzt, Maurice Auslander, W. L. Ayres, F. E. Baker, B. J. Ball, N. H. Ball, R. P. Bambah, Wallace E. Barnes, I. A. Barnett, R. G. Bartle, R. W. Bass, G. E. Bates, H. F. Becksfort, E. G. Begle, T. J. Benac, A. A. Bennett, F. D. Bennett, Stefan Bergman, Lipman Bers, E. E. Betz, R. H. Bing, Z. W. Birnbaum, I. E. Block, H. W. Bode, Raoul Bott, T. A. Botts, S. G. Bourne, J. W. Bower, J. W. Brace, Joseph Bram, L. D. Bram, H. W. Brinkmann, A. R. Brown, Jr., Leon Brown, F. H. Brownell, C. E. Burgess, R. S. Burington, J. H. Bushey, Jewell H. Bushey, G. H. Butcher, P. L. Butzer, S. S. Cairns, Eugenio Calabi, W. R. Callahan, E. A. Cameron, R. H. Cameron, H. H. Campaigne, J. F. Canu, L. V. Carlton, R. C. Carson, H. C. Carter, W. B. Carver, J. W. Cell, E. W. Cheney, P. L. Chessin, Joshua Chover, W. L. Chow, D. E. Christie, J. L. Chung, G. F. Clanton, C. E. Clark, F. E. Clark, F. M. Clarke, G. R. Clements, A. H. Clifford, E. W. Coffin, E. J. Cogan, I. S. Cohen, L. W. Cohen, Teresa Cohen, Harvey Cohn, Nancy Cole, A. H. Copeland, Sr., W. H. H. Cowles, V. F. Cowling, M. J. Cox, J. B. Crabtree, Frederic Cunningham, J. H. Curtiss, Chandler Davis, M. D. Davis, R. L. Davis, B. V. Dean, R. A. Dean, L. J. Deck, Allen Devinatz, S. F. Dice, R. C. DiPrima, J. L. Doob, F. G. Dressel, Nelson Dunford, W. L. Duren, Jr., William H. Durfee, W. F. Eberlein, R. P. Eddy, H. P. Edmundson, Gertrude Ehrlich, C. C. Elgot, Joanne Elliott, Arthur Erdélyi, Paul Erdös, D. H. Erkiletian, Jr., W. H. Fagerstrom, Michael Fekete, William Feller, F. G. Fender, F. A. Ficken, N. J. Fine, R. S. Finn, W. T. Fishback, Harley Flanders, B. A. Fleishman, W. H. Fleming, E. E. Floyd, S. A. Foote, L. R. Ford, L. R. Ford, Jr., H. A. Forrester, M. K. Fort, Jr., Tomlinson Fort, A. H. Fox, W. C. Fox, J. S. Frame, L. K. Frazer, Gerald Freilich, F. N. Frenkiel, C. H. Frick, R. E. Fullerton, A. S. Galbraith, H. L. Garabedian, I. E. Gaskill, L. D. Gates, H. M. Gehman, J. J. Gehrig, J. J. Gergen, Murray Gerstenhaber, J. H. Giese, Edwin Gillette, Leonard Gillman, Wallace Givens, A. M. Gleason, Casper Goffman, V. D. Gokhale, Michael Goldberg, Oscar Goldman, R. A. Good, A. W. Goodman, Basil Gordon, Saul Gorn, Morikuni Goto, W. H. Gottschalk, E. C. Gras, L. W. Green, Simon Green, F. L. Griffin, J. S. Griffin, Jr., Emil Grosswald, P. E. Guenther, V. H. Haag, Felix Haas, V. B. Haas, Sister M. Raphael Hafner, Franklin Haimo, E. E. Hammond, Jr., M. E. Hamstrom, L. S. Hart, Philip Hartman, H. H. Hartzler, Melvin Hausner, E. V. Haynsworth, E. A. Hedberg, G. A. Hedlund, Alex Heller, Melvin Henriksen, R. T. Herbst, Coleman Herpel, I. R. Hershner, Jr., I. N. Herstein, P. S. Herwitz, I. I. Hirschman, Jr., F. E. P. Hirzebruch, A. J. Hoffman, J. E. Hoffman, S. P. Hoffman, F. E. Hohn, B. W. Holz, J. G. Horne, 
Jr., E. M. Hove, Alfred Huber, G. B. Huff, Ralph Hull, D. W. Hullinghorst, L. S. Hunter, W. A. Hurwitz, M. A. Hyman, J. R. Isbell, R. F. Jackson, S. B. Jackson, H. G. Jacob, Jr., Arno Jaeger, R. E. Johnson, B. W. Jones, F. B. Jones, M. L. Juncosa, Mark Kac, Hyman Kamel, A. F. Kay, N. D. Kazarinoff, John Keesee, M. E. Kellar, L. M. Kells, J. B. Kelly, L. M. Kelly, J. H. B. Kemperman, M. R. Kenner, J. R. F. Kent, D. E. Kibbey, C. C. Kilby, Jr., George Klein, J. R. Kline, Fulton Koehler, T. L. Koehler, H. L. Krall, J. B. Kruskall, Jr., R. R. Kuebler, Jr., Harold W. Kuhn, S. H. Lachenbruch, Serge Lang, Harry Langman, Leo Lapidus, E. H. Larguier, C. G. Latimer, Patrick Leehey, Solomon Lefschetz, Marguerite Lehr, R. A. Leibler, Walter Leighton, R. B. Leipnik, W. W. Leontief, Marie Lesnick, J. J. Levin, Norman Levinson, A. L. Lewis, D. C. Lewis, Jr., S. D. Liao, H. M. Lieberstein, F. W. Light, Jr., T. P. G. Liverman, Charles Loewner, Lee Lorch, F. W. Lott, D. B. Lowdenslager, L. L. Lowenstein, C. I. Lubin, Eugene Lukacs, R. C. Lyndon, V. O. McBrien, B. H. McCandless, S. L. McDonald, E. A. McDougle, R. W. McKelvey, J. D. McKnight, Brockway McMillan, S. S. McNeary, E. J. McShane, C. C. MacDuffee, G. R. MacLane, Saunders MacLane, H. M. MacNeille, W. G. Madow, Wilhelm Magnus, Lawrence Markus, M. H. Martin, P. E. Martin, C. W. Mathews, A. P. Mattuck, K. O. May, F. M. Mears, A. E. Meder, Jr., Herman Meyer, L. F. Meyers, A. N. Milgram, Joseph Milkman, D. D. Miller, F. H. Miller, A. K. Mitchell, Don Mittleman, Deane Montgomery, A. H. Moore, T. W. Moore, W. E. Moore, W. K. Morrill, C. W. Munshower, F. D. Murnaghan, C. H. Murphy, Jr., F. H. Murray, G. G. Murray, W. R. Murray, Leopoldo Nachbin, L. W. Neustadt, August Newlander, Jr., Morris Newman, C. V. Newsom, R. Z. Norman, A. B. Novikoff, C. O. Oakley, G. G. O'Brien, R. E. O'Donnell, M. W. Oliphant, Gloria Olive, L. F. Ollman, E. M. Olson, Steven Orey, Morris Ostrofsky, J. C. Oxtoby, K. L. Palmquist, O. O. Pardee, Emanuel Parzen, G. W. Patterson, L. E. Payne, E. J. Pellicciaro, J. L. Penez, G. W. Petrie, III, B. J. Pettis, H. P. Pettit, Sister M. Socorro Piccirillo, Everett Pitcher, R. L. Plunkett, Harry Polachek, J. C. Polley, G. B. Price, M. H. Protter, F. M. Pulliam, A. L. Putnam, C. R. Putnam, Howard Raiffa, O. J. Ramler, J. F. Randolph, L. T. Ratner, G. E. Raynor, C. J. Rees, M. S. Rees, Haim Reingold, Russell Remage, C. N. Reynolds, D. E. Richmond, L. A. Ringenberg, R. K. Ritt, T. J. Rivlin, J. E. Robinson, L. B. Robinson, J. C. Rogers, I. H. Rose, J. B. Rosen, Saul Rosen, R. A. Rosenbaum, P. C. Rosenbloom, Herman Rubin, Phyllis Rubin, M. E. Rudin, Walter Rudin, H. E. Salzer, Hans Samelson, J. H. Sampson, James Sanders, Leo Sario, S. S. Saslaw, W. K. Saunders, J. B. Scarborough, Herbert Scarf, H. M. Schaerf, Robert Schatten, J. A. Schatz, A. E. Scheerer, S. A. Schelkunoff, E. V. Schenkman, E. C. Schlesinger, I. J. Schoenberg, A. L. Schurrer, Abram Schwartz, Sol Schwartzman, G. E. Schweigert, C. H. W. Sedgewick, Raymond Sedney, C. W. Seekins, I. E. Segal, G. B. Seligman, J. B. Serrin, J. M. Shaheen, Henry Sharp, Jr., Seymour Sherman, A. L. Shields, K. S. Shih, Marlow Sholander, Harold Shulman, J. A. Silva, L. L. Silverman, M. F. Smiley, R. F. Smith, Ernst Snapper, J. L. Snell, J. J. Sopka, D. E. Spencer, V. E. Spencer, C. A. Spicer, George Springer, E. R. Stabler, C. J. Standish, J. M. Stark, M. E. Stark, E. P. Starke, I. A. Stegun, C. F. Stephens, Rothwell Stephens, S. Z. Sternberg, F. M. Stewart, R. W. Stokes, R. R. Stoll, W. J. Strange, P. M. Swingle, Gabor Szegö, C. T. Taam, T. T. Tanimoto, Olga Taussky-Todd, M. E. Taylor, William Clare Taylor, Feodor Theilheimer, W. R. Thickstun, Jr., G. B. Thomas, Jr., J. M. Thomas, G. L. Thompson, D. L. Thomsen, Jr., R. M. Thrall, J. A. Tierney, E. W. Titt, John Todd, M. M Torrey, J. I. Tracey, C. J. Tremblay, A. W. Tucker, J. L. Ullman, J. L. Vanderslice, H. E. Vansant, A. H. Van Tuyl, M. C. Waddell, D. H. Wagner, R. J. Walker, A. D. Wallace, J. A. Ward, W. H. Warner, Gerard Washnitzer, Daniel Waterman, G. C. 
Webber, J. V. Wehausen, H. F. Weinberger, Morris Weisfeld, B. A. Welch, John Wermer, D. W. Western, F. J. Weyl, George Whaples, C. R. White, G. W. Whitehead, K. B. Whitehead, P. M. Whitman, Hassler Whitney, G. T. Whyburn, L. S. Whyburn, W. M. Whyburn, H. H. Wicke, D. V. Widder, A. B. Willcox, E. S. Williams, M. E. Williams, R. F. Williams, W. L. Williams, Alberta Wolfe, N. Z. Wolfsohn, K. G. Wolfson, Arthur Wouk, J. W. Wrench, Jr., F. B. Wright, Jr., F. M. Wright, Hidehiko Yamabe, D. M. Young, Jr., L. C. Young, Arthur Zeichner, J. A. Zilber.

The Twenty-seventh Josiah Willard Gibbs Lecture, entitled Mathematics in economics, was delivered by Professor Wassily Leontieff of Harvard University on Monday evening. Professor G. T. Whyburn, President of the Society, presided.

The Society was addressed by Professor A. D. Wallace of Tulane University on the Structure of topological semi-groups on Monday afternoon and by Professor D. V. Widder of Harvard University on Convolution transforms on Tuesday afternoon upon invitation of the Committee to Select Hour Speakers for Annual and Summer Meetings. At the former address Professor Deane Montgomery presided and Professor Leon W. Cohen presided at the latter.

The membership and their guests were entertained at tea in Levering Hall on Monday afternoon by the ladies of the Department of Mathematics of the University. Arrangements were made for visits to Annapolis on Tuesday and to places of interest in Baltimore on Wednesday.

On Wednesday evening, at the banquet under the auspices of both the Society and the Association, an address was delivered by Dr. Alan T. Waterman, Director of the National Science Foundation, in which he discussed the program of the Foundation in the mathematical sciences. Professor D. C. Lewis of the Johns Hopkins University was the toastmaster. A resolution expressing the appreciation of the Society and the Association to the Johns Hopkins University and to all who contributed their personal efforts to the success of the meeting was moved by Professor Ralph Hull and adopted by a rising vote.

On Wednesday afternoon December 30, 1953 the Bôcher prize was awarded to Professor Norman Levinson for the contributions to the theory of linear, nonlinear, ordinary and partial differential equations contained in his papers of recent years. These include studies on asymptotic behavior of solutions, stability questions, existence of periodic solutions, and perturbation theory. Professor Levinson then delivered a short address on the work for which he had been awarded this prize.

The annual Business Meeting of the Society was held on Wednes- 
day December 30,1953. The Secretary reported on the affairs of the Society.

At the annual election in which 958 votes were cast the following officers were elected.

President Elect, Professor R. L. Wilder.

Vice Presidents, Professors L. V. Ahlfors and Antoni Zygmund.

Associate Secretaries, Professors J. W. Green, J. H. Roberts, and J. W. T. Youngs.

Member of the Editorial Committee of the Bulletin, Professor W. T. Martin.

Member of the Editorial Committee of the Proceedings, Professor Richard Brauer.

Member of the Editorial Committee of the Transactions and Memoirs, Professor Herbert Busemann.

Member of the Editorial Committee of the Colloquium Publications, Professor A. A. Albert.

Member of the Editorial Committee of Mathematical Revierws, Professor R. P. Boas.

Member of the Editorial Committee of Mathematical Surveys, Professor Leo Zippin.

Representative on the Board of Editors of the American Journal of Mathematics, Professor Reinhold Baer.

Member of the Committee on Printing and Publishing, Dean C. J. Rees.

Members-at-large of the Council, Professors R. P. Dilworth, R. D. James, C. C. MacDuffee, D. V. Widder, and Antoni Zygmund.

Members of the Board of Trustees, Professors Einar Hille and $\mathrm{H}$. F. Bohnenblust.

The Secretary reported that as directed by the membership at a Business Meeting of the Society in Kingston, Ontario on September 1,1953 a statement concerning proposed amendments to the Bylaws had been included in the Programs for the November, 1953 meetings and had been sent to all members of the Society. In this statement the members were requested to write to the Secretary of the Society expressing opinions concerning these amendments. Thirty-two letters were received of which eighteen expressed approval and thirteen expressed disapproval. The Secretary reported that these letters had been considered by the Council and that the Council had voted to recommend again to the membership that these amendments to the By-laws be adopted and that the Council had requested the Secretary to read a statement concerning the attitude of the Council toward these amendments. The Secretary read 
the following statement:

In presenting these amendments, the Council is not asking for broader powers, nor that it be made easier for the Council to speak with respect to matters which affect the dignity and effective position of mathematics.

The Council believes, the world around us being what it is, that the Council will most probably have to consider such matters in the future.

The Council therefore wishes an orderly procedure to be established to ensure that action will be taken only after careful study and only with due deliberation.

The Council believes that these amendments guarantee such study and deliberation, and they are presented to you in this spirit.

After some discussion the members of the Society present at the Business Meeting voted 161 to 26 to adopt the following two amendments to the By-Laws:

1. To add to Article IV the following section:

Section 8: The Council shall also have power to speak in the name of the Society with respect to matters affecting the status of mathematics or mathematicians, such as proposed or enacted Federal or State legislation, conditions of employment in universities, colleges, or business, research or industrial organizations, regulations, policies or acts of governmental agencies or instrumentalities, and other items which tend to affect the dignity and effective position of mathematics.

With the exception noted in the next paragraph, a favorable vote of two-thirds of the entire membership of the Council, taken in accordance with the provisions of Section 4 of this Article, shall be necessary to authorize any statement in the name of the Society with respect to such matters. With the exception noted in the next paragraph, such a vote may be taken only if written notice shall have been given to the Secretary by the proposer of any such resolution not later than one month prior to the Council meeting at which the matter is to be presented, and the vote shall be taken not earlier than one month after the resolution has been discussed by the Council.

If, at a meeting of the Council, there are present members having a total of twelve votes, as defined in Section 4 of this Article, then the prior notification to the Secretary may be waived by unanimous consent. In such a case, a unanimous favorable vote by those present shall empower the Council to speak in the name of the Society.

The Council may also refer the matter to a referendum mail vote of the entire membership of the Society, and shall make such reference if a referendum is requested, prior to final action by the Council, by two hundred or more members. The taking of a referendum shall act as a stay upon Council action until the votes have been canvassed, and thereafter no action may be taken by the Council except in accordance with a plurality of the votes cast in the referendum.

2. To amend Section 1 of Article $X$ by replacing the last sentence of this article, "No matter of general business shall be considered at any meeting of the Society except the Annual Meeting, without the recommendation of the Council" by the following: 
There shall be a business meeting of the Society at the Annual Meeting and at the Summer Meeting. A business meeting of the Society shall take final action only on business accepted by unanimous consent, or business notified to the full membership of the Society in the call for the meeting. Such notification shall be made only when so directed by a previous business meeting of the Society or by the Council.

The Council met on Monday evening, December 29, 1953.

The Secretary announced the election of the following forty-two persons to ordinary membership in the Society:

Mr. Richard Logan Becker, Yale University;

Dr. Jacob Floyd Blackburn, U. S. Air Force, Institute for Advanced Study;

Mr. Thomas Roy Brahana, Dartmouth College;

Mrs. Ilse Brauer, Brandeis University;

Dr. William Egbert Briggs, University of Colorado;

Reverend Anthony Edmund Cahill, Belmont Abbey College;

Mr. George Charles Caldwell, University of North Carolina;

Mr. Alan Cobham, Navy Department, Washington, D. C.;

Dr. Margaret Frances Conroy, Purdue University;

Mr. Ralph Deutsch, Hughes Aircraft Company, Culver City, California;

Mr. Sylvan Howard Eisman, Frankford Arsenal, Philadelphia, Pennsylvania;

Dr. Pao-hsien Paul Fang, Catholic University of America;

Dr. Clemens Bernard Hanneken, Marquette University;

Mr. William Edward Hartnett, University of Kansas;

Mr. Walter Hoffman, Wayne University;

Professor George E. Hudson, College of Engineering, New York University;

Mr. Charles William Huff, University of Georgia;

Mr. Andrew McCampbell Jones, University of Kansas;

Professor Tosio Kato, University of Tokyo;

Mr. Clement Fisher Kent, Navy Department, Washington, D. C.;

Dr. David Carman Kleinecke, University of California, Berkeley;

Professor Joachim Lambek, McGill University;

Dr. Philip Joseph Leah, L'Immaculée Conception, Montreal, Quebec;

Professor Anthony Richard Lovaglia, San Jose State College;

Mr. Marvin David Marcus, University of California, Berkeley;

Mr. James Michael McLynn, Logistics Research Project, Washington, D. C.;

Mr. Robert Price Mitchell, University of Denver;

Mr. John Henry Monahan, Boston College;

Mr. Dewey Moore, Naval Ordnance Laboratory, White Oak, Maryland;

Dr. Paul Stallings Mostert, Tulane University;

Miss Mary Jane Palmerlee, Tulane University;

Mr. Jack Herbert Padgett, Armstrong College;

Mr. Lee Howard Pennington, Florida Agricultural and Mechanical College;

Mr. Robert George Pohrer, U. S. Armed Forces;

Professor Hanan Rubin, University of Tennessee;

Dr. William Richard Seugling, Allstates Engineering Company, Trenton, New Jersey;

Dr. Ray Orville Skinner, National Research Council, Ottawa, Ontario, Canada;

Miss Ruth Rebekka Struik, New York University;

Dr. Beat Andreas Troesch, New York University;

Mr. Charles Wyatt Williams, Retired Consulting Engineer; 
Professor Carvel Stewart Wolfe, Shepherd College;

Mr. Bernd Zondek, I. B. M. Corporation, New York, New York;

It was reported that the following one hundred fifty-three persons had been elected to membership on nomination of institutional members as indicated:

University of Alabama: Professor Holland C. Filgo, Jr.

University of British Columbia: Miss Charlotte Forese and Mr. Rimhak Ree.

Brooklyn College: Mr. Donald Solitar.

Brown University: Professor Paul Germain, Mr. Frank Pantaleone Palermo, and Professor Ronald S. Rivlin.

University of California, Berkeley: Mr. Donato Anthony D'Esopo, Mr. Thomas

Shelburne Ferguson, Mr. Marvin David Marcus, and Mr. Dana Stewart Scott.

University of California, Davis: Mr. Peter Jay Chrisler.

University of California, Los Angeles: Mr. Genovevo López, Mr. John L. Selfridge, Mr. Wayne Earl Smith, and Mr. Roy Takenaga.

Carnegie Institute of Technology: Professor Hirsh Gerald Cohen.

University of Chicago: Professor Florent Joseph Bureau, Mr. George Kolettis, Jr., Mr. Robert Harbison Mountjoy, Mr. Edward Charles Posner, Dr. Marcel Riesz, Mr. Alexandre Augusto Martins Rodriques, and Dr. Geoffrey Colin Shephard.

University of Cincinnati: Mr. Harvey M. Weitkamp.

City College of New York: Mr. Samuel Jacob Klein, and Mr. Menasha Joseph Tausner.

Columbia University: Mrs. Betty J. Flehinger, Mr. Bernard A. Fusaro, Mr. Bert Mendelson, Mr. Subramanian Sankaran, Mr. Harold R. Steele, and Mr. Bruce Travis Weber.

Cornell University: Mr. Robert McCallum Blumenthal, Mr. Kalman Joseph Cohen, Mr. George Edwin Collins, Mr. Leonard Evens, Mr. Angelo Margaris, Mr. Joel David Pincus, and Mr. Norman Stein.

Duke University: Mr. E. Warren Herron, Mr. John Herbert Hodges, and Mr. Robert Louis Padgett.

Haverford College: Mr. Robert T. Seeley.

Johns Hopkins University: Mr. Joseph Auslander, Mr. Robert I. Berg, Professor Carl F. Christ, Mr. Howard Charles Kerpelman, Mr. Marc L. Nerlove, Mr. Bernard Okun, Mr. Rolf R. Piekarz, Dr. Theodore J. Rivlin, Mr. Lee Suyemoto, and Mr. Richard Alan Swirnow.

University of Illinois: Mr. Peter George Braunfeld, Mr. William Carleton Healy, Jr., Mr. Charles Lewis Keller, Jr., Mr. Charles Neville Maxwell, Mr. Jack Unger Russell, Dr. Michio Suzuki, and Mr. John Eldon Whitesitt.

Indiana University: Mr. Norman George Meyers, and Mr. Walter Noll.

Institute for Advanced Study: Dr. Hirotada Anzai, Dr. Rene Deheuvels, Dr. Benoit B. Mandelbrot, Professor Kakutaro Morinaga, Dr. Gert Otto Sabidussi, Mr. John C. Shepherdson, and Dr. Kurt Strebel.

Iowa State College: Mr. William John Swartz.

University of Kansas: Mrs. Marta Mejía de Valle, and Mr. Elbert Abner Walker. Lehigh University: Mr. Ervin Kenneth Dorff.

Massachusetts Institute of Technology: Mr. Fred Gunther Brauer, Mr. A. Jay Goldstein, Mr. John Evans Kimber, Jr., Mr. Robert Francis McIntosh, Mr. James Thomas Morse, and Mr. Roger Mayfield Simons.

University of Michigan: Mr. John Mitchell Gary, Mr. Samuel Roscoe Knox, Mr 
John Paul Line, Mr. Robert Fred Lyjak, Mr. David Arne Storvick, Mr. Thomas Patrick Walker, Jr., and Mr. John Harris Walter.

Michigan State College: Mr. James Leland Bailey, Mr. William John Hardell, Mr. Bernard Jacobson, Dr. Delia Koo, Miss Mary Margaret Quinn, and Mr. Robert Earl Sechler.

University of Minnesota: Mr. Ernest Godfrey Kimme, Mr. Eugene James Putzer, and $\mathrm{Mr}$. Jerome $Z$. Yeh.

University of New Hampshire: Miss Evelyn Jean Jones.

New York University: Mr. Walter Koppelman, Mr. Herbert C. Kranzer, Mr. Walter Littman, and Mr. Nathan Newman.

Northwestern University: Mr. John Martin Bachar, Jr., Mr. Mykola Marchenko, Jr., Mrs. Vera Stepen Pless, and Mrs. Elizabeth Maude Rowlinson.

Ohio State University: Mr. George Arthur Craft.

University of Oregon: Mr. LeRoy John Warren.

University of Pennsylvania: Mr. Hugh Norton Albright, Mr. Michael Rabin, and

Dr. Karl Zeller.

The Pennsylvania State University: Professor Sidney Foler Mack.

Purdue University: Mr. Theodore S. Chihara.

Queens College: Mr. Arthur Kaufman.

The Rice Institute: Mr. Horace Perry Flatt.

University of Rochester: Mr. Edward T. Wong.

Rutgers University: Professor Orhan H. Alisbah and Mr. Sufian Y. Husseini.

University of Southern California: Mr. Joel M. Alperin, Mr. A. V. Balakrishnan,

Dr. Julius Samuel Bendat, Mr. Howard Bryner Jenkins, Mr. H. Potter Kerfoot, Mr.

Ching-Hwa Meng, Mr. Allen Ralph Sims, Mr. Alton Hutchison Smith, and Mr.

Eugene Michael Zaustinsky.

Stanford University: Mr. Charles Ballantine, Mr. Joseph Omer Carter, Dr. James

Joseph McMahon, Captain Ralph Hugh Pennington, and Mr. Peter William Shaw.

Syracuse University: Mr. Rafael Van Severen Chacon, Mr. David Chedell Hiser,

Mrs. May E. Risch Kinsolving, Mr. William Eugene Kopka, and Mr. Luke Nicholas

Zaccaro.

University of Toronto: Mr. Douglas West Allan, Dr. Paul Carl Gilmore, Mr.

Albert Harold Lightstone, and Mr. Floyd Grant Robinson.

University of Utah: Mr. Louis Carl Barrett.

University of Virginia: Mr. Billey Joe Gilpin, Mr. William Lawrence Harkness,

Mr. James Louis Hatfield, Mr. Powhatan Moncure, Jr., and Mr. Henry Warren

Thwing.

University of Washington: Miss Thelma Mae Chaney and Professor Orval M. Klose.

Wellesley College: Miss Mary Louise Holton.

University of Wisconsin: Mr. John Edward Barger, Mr. William Robert Britten-

ham, Mr. Morton Brown, Mr. S. Ashby Foote, Mr. George Gioumousis, Mr. Robert

Leroy Glass, Mr. Harold Irwin Ottoson, Miss Catherine Shelton Standerfer, Mr. A. D.

Stewart and Mr. Richard E. Vesley.

Yale University: Mr. Alvin Norman Feldzamen, Mr. Bruno Harris, Mr. Stephen Elliott Puckette, and Mr. Earl Jay Taft.

The Secretary announced that the following had been admitted to the Society in accordance with reciprocity agreements with various mathematical organizations: Edinburgh Mathematical Society: Miss Nancy Walls, Edinburgh Mathematical Society; Indian Mathemat- 
ical Society: Miss Abha Mitra, Women's College, Patna, India; Islenzka Staeröfraeöafelagiö: Mr. Gumnar Bodvarsson, State Electricity Authority, Iceland; Schweizerische Mathematische Gesellschaft: Dr. Peter Henrici, American University (under contract with National Bureau of Standards); Wiskundig Genootschap: Professor Nicolaas Govert deBruijn, University of Amsterdam.

The Secretary is pleased to report at this time that the ordinary membership of the Society is now 4485 , including 436 nominees of institutional members and 38 life members. The membership of the Society continues to show a slow, but steady, growth. There are also 125 institutional members. The total attendance at all meetings in 1953 was 2748 ; the number of papers read was 790; there were 16 hour addresses, 4 Colloquium Lectures, 1 Gibbs Lecture, and 21 papers at the Applied Mathematics Symposium. The number of members attending at least one meeting was 1600 .

The following appointments by the President were reported: as a committee to nominate a representative of the Society on the Policy Committee for Mathematics: Deane Montgomery, Chairman, A. A. Albert and E. R. Kolchin; as a committee to cooperate with a Committee of the Mathematical Association of America on a Placement Service: Leon W. Cohen, Chairman, and James A. Clarkson; as a committee to nominate the award of the Cole Prize in Algebra: Oscar Zariski, Chairman, Richard Brauer and C. C. MacDuffee; as a Committee on Arrangements for the Summer Meeting in Laramie, Wyoming, August 30-September 3, 1954: C. F. Barr, Chairman, H. M. Gehman, J. W. Green, Greta Neubauer, O. H. Rechard, Nathan Schwid, S. R. Smith, W. N. Smith, P. O. Steen and V. J. Varineau; as a member of the Committee on Places of Meetings for a term of three years beginning January 1, 1954: Professor W. M. Whyburn (Committee consists of Professors M. F. Smiley, Chairman, E. R. Lorch and W. M. Whyburn); as a member of the Committee on Visiting Lectureships for a term of three years beginning January 1, 1954: Professor Paul Halmos (Committee now consists of Professors Garrett Birkhoff, Chairman, Einar Hille and Paul Halmos); as Chairman of the Committee on Applied Mathematics: F. J. Murray; and as members for terms of three years beginning January 1, 1954: R. S. Burington and R. E. Bellman; as members of the Committee to Select Hour Speakers (terms to expire December 31, 1956): Summer and Annual Meetings: Wilfred Kaplan (Committee now consists of E. G. Begle, Chairman, Oscar Zariski and Wilfred Kaplan); Eastern Sectional Meetings: Nelson Dunford (Committee now consists of L. W. Cohen, Chairman, Nelson Dunford and A. 
M. Gleason); Southeastern Sectional Meetings: W. V. Parker (Committee now consists of J. H. Roberts, Chairman, J. J. Gergen and W. V. Parker); Western Sectional Meetings: M. E. Shanks (Committee now consists of J. W. T. Youngs, Chairman, E. E. Moise and M. E. Shanks); Far Western Sectional Meetings: J. L. Kelley (Committee now consists of J. W. Green, Chairman, R. D. James and J. L. Kelley); as tellers for the 1953 annual election: W. S. Massey and F. M. Stewart.

The following appointments to represent the Society were reported: at the inauguration of Perry Epler Gresham as President of Bethany College on October 23, 1953: Professor Bradford Tye; at the inauguration of Clarence B. Hilberry as President of Wayne University on Monday, November 9, 1953: Professor T. H. Hildebrandt; at the Seventy-fifth Anniversary of the founding of Duquesne University, November 11 and 12: Professor J. S. Taylor.

The Secretary reported that Professor Paul White had accepted an invitation to deliver an hour address at the May 1954 meeting at Yosemite National Park and that Professor Enrico Fermi had accepted an invitation to deliver the Gibbs Lecture in 1954.

The Council approved the substitution of Professor E. R. Kolchin as a representative of the Society on the Editorial Board of the American Journal of Mathematics for the academic year 1953-54.

The Council voted to establish a new periodical to be called Notices of the American Mathematical Society to be under the editorial supervision of the Editorial Committee for the Bulletin and to contain programs of meetings, the notes and the list of new publications which now appear in the Bulletin as well as special notices to members of the Society.

The Council approved the following times and places of sectional meetings in 1954: February 27 at Hunter College, New York City; April 30 and May 1 at the University of Chicago; May 1 at Yosemite National Park; October 30 at Massachusetts Institute of Technology.

The Council voted to accept an invitation to hold the 1954 annual meeting December 27-29 at the University of Pittsburgh.

The Bulletin Editorial Committee reported that the journals used 622 pages in 1953. The Council voted to recommend to the Board of Trustees that the Bulletin be authorized for 1954 to print 625 pages less the number of pages of notes and lists of new periodicals which would be transferred to the Notices.

The Transactions and Memoirs Editorial Committee reported that the interval between receipt of a manuscript and publication is approximately one year. The Council voted to recommend to the 
Trustees that 1100 pages be published in the Transactions for 1954 . The Mathematical Reviews Editorial Committee reported that 1150 pages had been published in 1953. The subscription list as of November, 1953 was 2287.

The Proceedings Editorial Committee reported that the interval between receipt of a manuscript and publication was approximately eight months. The Council voted to recommend to the Board of Trustees that 1000 pages be authorized for the 1954 Proceedings. Professors Bernard Friedman, W. H. Gottschalk, Marshall Hall, and J. A. Jenkins were reported as new assistant editors for the Proceedings.

On recommendation of the Colloquium Editorial Committee the Council voted to invite Professor Nathan Jacobson to deliver the 1955 Colloquium Lecture.

The Council voted to co-sponsor with the Office of Ordnance Research a Second Symposium in Applied Mathematics at the University of Chicago on April 29 and 30, 1954.

The Council voted with the cooperation of the Mathematical Association of America to establish an Employment Register. This Register will consist of notices from academic institutions, industrial firms, and government agencies of vacant positions. These notices will be available for inspection at the Summer and Annual Meetings and at the April Meetings.

The Council adopted the following resolution:

The Council of the American Mathematical Society here takes cognizance of the resignation this past September of Dr. Mina Rees as Head of the Mathematics Section of the Office of Naval Research. She has accepted a position as Dean of the Faculty at Hunter College. We congratulate Hunter College on this wise selection and can only say that our heavy loss as mathematicians is the gain of Hunter College.

The very striking and brilliant contributions made by pure (non-military, nonapplied) science, not least of these by mathematics, to the winning of World War II is well known. It was clearly seen by the government and those responsible for the armed services that a large scale fostering by the U. S. government of fundamental research, the basis of all research, was unavoidable. Only thus could we hope to hold our own in years to come, and incidentally build up a suitable reserve of talented men for emergencies. This was actually acted upon by the Navy who thus took the lead by some years with the creation of the Office of Naval Research. Needless to say as the purest of all sciences, mathematical research might well have lagged behind in such an undertaking. That nothing of the sort happened is beyond any doubt traceable to one person-Mina Rees. Under her guidance, basic research in general, and especially in mathematics, received the most intelligent and wholehearted support. No greater wisdom and foresight could have been displayed and the whole postwar development of mathematical research in the United States owes an immeasurable debt to the pioneer work of the Office of Naval Research and to the alert, vigorous and farsighted policy conducted by Miss Rees. The influence of these policies has been such 
that it vitally affected later developments: the activities of Air Force and Ordnance research, the National Science Foundation itself. It is well known that in these more recent organizations Mina Rees was constantly appealed to for counsel and guidance.

As Miss Rees leaves her task, the Council of the American Mathematical Society desires to express to her in the name of the whole mathematical community its warmest feelings of appreciation for her past performance and extends to her its best wishes for the future.

There were fifteen sessions for contributed papers, including two sessions for late papers, presided over by Professors I. A. Barnett, V. F. Cowling, L. R. Ford, R. E. Fullerton, Casper Goffman, G. B. Huff, W. A. Hurwitz, R. E. Johnson, Saunders MacLane, A. N. Milgrim, P. C. Rosenbloom, Ernst Snapper, E. W. Titt, A. D. Wallace, W. M. Whyburn. The abstracts of the papers are appended. Those having the letter " $t$ " following the abstract number were read by title. Where a paper, presented in person, has more than one author, the symbol (p) follows the name of the author who presented it. Professor Hanan Rubin was introduced by Professor J. J. Stoker, Dr. Shimada and Dr. Uehara by Professor W. S. Massey, Mr. S. I. Plotnik by Professor Orrin Frink, Dr. P. S. Mostert by Professor S. T. Hu, Professor G. E. Uhlenbeck and Mr. G. W. Ford by Professor Frank Harary, Mr. J. H. Monahan by Professor H. G. Haefeli, Professor Hao Wang by Mr. Joel Pitcairn, Dr. W. B. Jurkat by Professor C. N. Moore, Mr. W. J. Feeney by Professor O. J. Ramler, and Professor John Nash, Dr. Schwartz, Captain R. H. Pennington and Mr. J. H. Case by Professor Leon W. Cohen.

\section{Algebra ANd Theory of Numbers}

177t. S. A. Amitsur: Differential polynomials and division algebras. I. The resultant of differential polynomials.

Let $F[t]$ be the ring of differential polynomials in $t$ with multiplication defined by $a t=t a+a^{\prime}$, where $a \rightarrow a^{\prime}$ is a derivation in a field $F$ of characteristic zero. Using a correspondence between differential operators and differential polynomials (Jacobson, Ann. of Math. vol. 38 (1937) pp. 484-507), make correspond to any two polynomials $p(t), q(t)$ of degrees $n$ and $m$ a polynomial $p(t) \times q(t)$ of degree $n m$. This new operation $X$ is proved to be associative, commutative, and distributive with respect to multiplication and decomposition between classes of similar polynomials. Let $p(t)=\sum t^{\nu} a_{\nu}$, define the adjoint polynomial $p^{*}(t)=\sum a_{\nu}(-t)^{\nu}$, and the nullity of $p(t)$ to be the maximal number of independent solutions in $F$ (over the field of constants) of the linear differential equation $\sum a_{\nu} z^{(\nu)}=0$. With these definitions it is shown: (1) A necessary and sufficient condition that there exists a factorization $p(t)=p_{1}(t) p_{2}(t), q(t)=q_{1}(t) q_{2}(t)$ such that $p_{1}(t)$ is a non-constant polynomial similar to $q_{2}(t)$ is that the nullity of $p(t) \times q^{*}(t)$ be $>0$. (In a special case this can be shown to yield the well known result on the resultant of commutative polynomials.) Refer to $p(t) \times q^{*}(t)$ as the resultant of $p(t)$ and $q(t)$. (2) The nullity of $p(t) \times p^{*}(t)$ is exactly the dimension of the invariant ring of $p(t)$, that is: the ring of all classes of $F[t] / p(t) F[t]$ whose representatives $q(t)$ satisfy $q(t) p(t) \equiv 0 \bmod p(t) F[t]$. (The finite- 
ness of this dimension was shown by Ore, see also Jacobson, ibid. p. 507.) (Received November 12, 1953.)

\section{S. A. Amitsur: Differential polynomials and division algebras.}

\section{Central simple algebras.}

Let $C$ be a field of characteristic zero algebraically closed in an extension field $F$. Define in $F$ a derivation: $a \rightarrow a^{\prime}$ with $C$ as the field of constants. Using the notation of the preceding abstract, a polynomial $p(t)$ in $F[t]$ of degree $n$ is called an $A$-polynomial if the nullity of $p(t) \times p^{*}(t)$ is $n^{2}$. The main results obtained are: (1) The invariant ring of an $A$-polynomial $p(t)$ of degree $n$ is a central simple algebra $A_{p}$ of order $n^{2}$ over $C$ and is split by $F$; conversely, every central simple algebra of order $n^{2}$ over $C$ which is split by $F$ is isomorphic to some invariant ring $A_{p}$ of an $A$-polynomial $p(t)$ of degree $n$. In particular, the algebra is a division algebra if and only if the corresponding $A$-polynomial is an irreducible polynomial. (2) $A_{p} \times A_{q} \cong A_{p \times q}$. (3) If $p(t)=\sum t^{\nu} a_{\nu}$ is an $A$-polynomial of degree $n$ then the set of all solutions of the differential equation $\sum a_{\nu} z^{(\nu)}=0$ has a basis of the form $x_{i} y^{1 / m}, i=1, \cdots, n$, where $x_{i}, y \in F(D)$ with $D$ any splitting field of $A_{p}$, and where $m$ is the exponent of $A_{p}$. As an application of the preceding results it is shown that: if $D$ is a normal extension of $C$ with a Galois group $G$, then the Brauer group of all division algebras split both by $F$ and $D$ is isomorphic with the first cohomology group of $G$ with coefficients in the multiplicative group $[F(D)]^{*} / D^{*}$ where $\left(^{*}\right)$ indicates the multiplicative group of all nonzero elements. The last result is obtained also by methods of the cohomology theory of groups. (Received November 12, 1953.)

\section{N. C. Ankeny: Quadratic forms in quadratic real fields.}

One defines certain fundamental domains for Hilbert modular groups associated with a quadratic real extension of the rationals. One shows that certain group extensions are necessary if the class number of the field is greater than 1 . One then applies these results to representations of numbers by quadratic forms. This is a generalization of certain results of Maass. (Received October 21, 1953.)

180t. H. W. Becker: Maverick and parametric solutions of $\epsilon^{2} \zeta^{2}-\eta^{2} \theta^{2}$, $\epsilon^{2} \eta^{2}-\zeta^{2} \theta^{2}, \epsilon^{2} \theta^{2}-\zeta^{2} \eta^{2}=s$.

Euler proposed this problem, Dickson's History II, p. 661-662, and came up with one solution: $25,12,39,20$. But a solution is deducible from any triad of Petrus vectors with common index. Thus from $\left(59^{4}-58^{4}\right)\left\{\left(9^{4}-7^{4}\right)\right.$ or $\left.\left(11^{4}-2^{4}\right)\right\}=s$ one gets the solutions: $185,12,39,148$ and $925,117,60,444$. Triadizing with $137^{4}-7^{4}$ (also of index 65) one gets 97, 48, 780, 388;485, 468, 300, 291; and 3589, 468, 2220, 291. From $\left(31^{4}-27^{4}\right)\left\{\left(7^{4}-3^{4}\right)\right.$ or $\left.\left(17^{4}-1\right)\right\}=|s|$ one gets the solutions $65,29,24,78$ and $654,264,319,545$. Triadizing with $271^{4}-213^{4}$ (also of index 145) one gets the solutions $545,319,218,88$ and $1308,638,1090,528$. The foregoing are all mavericks, as to this problem. But as by-products of P.V. forms there are the parametric solutions: $[\epsilon=\eta, \zeta, \theta]=\left[r^{4}+s^{4}, 2 r s\left(r^{2} \pm s^{2}\right)\right] ; \quad\left[r^{8}+20 r^{6} s^{2}-26 r^{4} s^{4}+20 r^{2} s^{6}+s^{8}, 2\left(r^{4}-6 r^{2} s^{2}+s^{4}\right)\right.$ $\left.\left\{4 r s\left(r^{2}-s^{2}\right), \quad\left(r^{2}+s^{2}\right)^{2}\right\}\right] ; \quad\left[\left(r^{2}-s^{2}\right)\left(r^{8}-4 r^{6} s^{2}-6 r^{4} s^{4}-4 r^{2} s^{6}+s^{8}\right), \quad\left(r^{2} \pm s^{2}\right)\left(r^{8}+4 r^{6} s^{2}\right.\right.$ $\left.\left.-6 r^{4} s^{4}+4 r^{2} s^{6}+s^{8}\right)\right] ; \quad\left[\Pi\left(9 r^{4} \pm 6 r^{3} s+2 r^{2} s^{2} \pm 2 r s^{3}+s^{4}\right), \quad 4 r s\left(r^{2}-s^{2}\right)\left(9 r^{2}-s^{2}\right)\left(3 r^{2}+s^{2}\right)\right.$, $\left.\left(9 r^{4} \pm 6 r^{3} s+2 r^{2} s^{2} \pm 2 r s^{3}+s^{4}\right)\right]$. The latter is the Hillyer case of any Carmichael vector in which $2 a b\left(a^{2}-b^{2}\right)=2 c d\left(c^{2}-d^{2}\right)=y^{1 / 2},\left(a^{2}+b^{2}\right) \cdot\left(c^{2}+d^{2}\right)=t_{1} t_{2}=t$. In terms of such C.V., $[\epsilon=\eta, \zeta, \theta]=\left[t, y, t_{1}^{2}\right.$ or $\left.t_{2}^{2}\right]$; this is equivalent to a Petrus transform, with 
$y\left(t_{2}^{2} \pm t_{1}^{2}\right)=2 u v, 2 x z$; these also constitute a Hero $\Delta$ of 2 sq. sides $t_{1}^{2}, t_{2}^{2}$ and alt. $y$. (Received November $16,1953$.

\section{1t. H. W. Becker: O'Riordan conjugates of rational cuboids.}

Let $x^{2}+y^{2}=u^{2}, y^{2}+z^{2}=v^{2}, x^{2}+z^{2}=w^{2}$. Utilize O'Riordan's forms, Dickson's History II, p. 504, under $C, \alpha$ or $\delta, B, \beta, \gamma, A$ or $D \rightarrow x, y, z, u, v, w$. The resulting quadratics are $e / f=\left[3 a b\left(c^{2}-d^{2}\right)+c d\left(a^{2}-b^{2}\right) \pm 2 \Delta_{c}^{1 / 2}\right] /(a d-b c)^{2}, \quad \Delta_{e}=2 a b\left(c^{2}-d^{2}\right)(a c-b d)$ $(a d+b c)$; same, under $e, f \leftrightarrow a, b ; c / d=\left[(a e-b f)(a f+b e) \pm 2 \triangle_{a}^{1 / 2}\right] /\left[(a f-b e)^{2}-4 a b e f\right]$, $\Delta_{c}=2 a b e f\left\{(a e+b f)^{2}-(a f-b e)^{2}\right\}$. The forms $\Delta=\square$ are a new approach to rational cuboids. Moreover, from a known cuboid $K$, if reducible to O'Riordan's forms via $v \pm z, u \pm x$, etc., 3 new cuboids may be found, the conjugates of $K$ under change of sign of $\Delta_{a, c, e}^{1 / 2}$. From each of these 2 more may be found, etc. ad inf. Thus in the Rignaux-Crussol parametric solution, $a, b ; c, d ; e, f=(2 r-s)(r+2 s)(r-s)^{2}, r s(r-3 s)^{2}$; $r(2 r-s), s(2 r+s) ; r(r+2 s)\left(r^{2}+s^{2}\right), 2 s(2 r-s)\left(r^{2}-r s-s^{2}\right)$, the first set of conjugates is: $a^{\prime}, b^{\prime} ; c^{\prime}, d^{\prime} ; e^{\prime}, f^{\prime}=(2 r-s)(r+2 s)\left(r^{3}+r^{2} s+4 r s^{2}+2 s^{3}\right)^{2}, r s\left(7 r^{3}+r^{2} s+2 s^{3}\right)^{2} ;(c, d)$ (12th degree polynomials); $e\left(2 r^{2}-6 r s+5 s^{2}\right)^{2}, f s^{2}(2 r-s)^{2}$. Here the 1 st and $3 \mathrm{~d}$ conjugates are equivalent, and both cover nos. 1, 19 and 20 of Kraitchik's table, Scripta Mathematica vol. $11(1945)$ p. 326, in which 41 mavericks now remain. In the classic Saunderson solution, $a, b ; c, d ; e, f=\left(r^{2}+s^{2}\right)^{2}, 8 r s\left(r^{2}-s^{2}\right) ; r, s ; r, s$, the 1 st set of conjugates being: (degenerate); $r\left(r^{8}+52 r^{6} s^{2}+118 r^{4} s^{4}-176 r^{2} s^{6}+17 s^{8}\right)$, same under $r \leftrightarrow s ; r\left(r^{4} 10 r^{2} s^{2}-7 s^{4}\right)^{2}$, same under $r \leftrightarrow s$. (Received November 16, 1953.)

182t. Leonard Carlitz: Congruence properties of special elliptic functions.

In a previous paper: The coefficients of singular elliptic functions (to appear in Math. Ann.) the writer derived certain divisibility properties of the coefficients of sn $x$ and $x /$ sn $x$ when the function admits of complex multiplication. In the present paper it is assumed that the modulus $k^{2}$ satisfies $W_{p}\left(k^{2}\right)=0$, where $W_{p}(u)$ $=\sum_{s=0}^{m}\left({ }_{s}^{m}\right)^{2} u^{s}$ and $p=2 m+1$ is a prime. Results similar to but somewhat less precise than those in the singular case are obtained. For example if sn $x=\sum \alpha_{m} x^{m} / m$ !, then $\alpha_{m} \equiv 0\left(\bmod p^{r}\right)$ for $m \geqq p r$; if $x /$ sn $x=\sum \beta_{m} x^{m} / m !$, then $\beta_{m} \equiv 0\left(\bmod p^{r+t}\right)$ for $m>p r, p-1 \nmid m, p^{t} \mid m$, while if $p-1 \mid m$ then $\beta_{m} \equiv 0\left(\bmod p^{r-1}\right)$. The coefficients of $(x / \operatorname{sn} x)^{h}, 1 \leqq h<p-1$, are also discussed. The polynomial $W_{p}(u)$ is related to the Legendre polynomial; a number of results on the factorization of $W_{p}(u)(\bmod p)$ are obtained. (Received November 14, 1953.)

183t. Leonard Carlitz: The number of solutions of a special quadratic congruence.

Let $p$ be an odd prime and $m, r, t \geqq 1$. Let $A=\left(a_{i j}\right)$ be a square matrix of order $m$, where the $a_{i j}$ are integers such that $\operatorname{det} A \neq 0(\bmod p)$. Making use of some results of Siegel on the matric congruence $X^{\prime} A X \equiv B\left(\bmod p^{r}\right)$ (Ann. of Math. (2) vol. 36 (1935) pp. 527-606), one determines the number of solutions $X$ of the congruence $\left|\begin{array}{l}A X \\ X^{\prime} 0\end{array}\right| \equiv b\left(\bmod p^{r}\right)$, where $b \neq 0\left(\bmod p^{r}\right)$ and $X$ is an $m \times t$ matrix. (Received November 14, 1953.)

184t. Leonard Carlitz: The number of solutions of some equations in a finite field.

The types of equations discussed are (1) $Q\left(\xi_{1}, \cdots, \xi_{2 r+1}\right)=g\left(\eta_{1}, \cdots, \eta_{s}\right)$ where $Q$ denotes a quadratic form with coefficients in $G F\left(p^{n}\right)$ and the polynomial $g(\eta)$ is a 
product of quadratic, cubic, or quartic polynomials of certain kinds. Application is made of results on Jacobsthal sums. (2) As in (1), with $g(\eta)=\prod_{i=1}^{i}\left(\eta_{i}^{p}-\eta_{i}+\alpha_{i}\right)$. (3) $\sum_{i=1}^{:} \alpha_{i}\left(\eta_{i}^{p}-\eta_{i}\right)=\alpha$ and $Q(\xi)=\sum_{i=1}^{i} \alpha_{i}\left(\eta_{i}^{p}-\eta_{i}\right)+\alpha$. (4) $\xi^{f}=\eta^{p}-\eta+\alpha$, where $f \mid p^{n}-1$. (Received November 14, 1953.)

\section{5t. A. H. Clifford: Bands of semigroups.}

A semigroup $S$ is called a band of semigroups of type $\mathcal{T}$ if it is the class sum of a set of mutually disjoint subsemigroups $S_{\alpha}(\alpha \in I)$, each of type $\mathcal{T}$, such that the product $S_{\alpha} S_{\beta}$ of any two of them is wholly contained in a third: $S_{\alpha} S_{\beta} \subseteq S_{\gamma} . S$ is called a semilattice of semigroups of type $\mathcal{T}$ if $S_{\alpha} S_{\beta}$ and $S_{\beta} S_{\alpha}$ are both contained in the same $S_{\gamma} . S$ is called a matrix of semigroups of type $\mathcal{T}$ if the index class $I$ is a direct product $J \times K$ of two classes $J$ and $K$, and if $S_{i \kappa} S_{j \lambda} \subseteq S_{i \lambda}$; for all $i, j \in J$ and all $\kappa, \lambda \in K$. It is shown that a band of semigroups of type $\mathcal{T}$ is a semilattice of semigroups each of which is a matrix of semigroups of type $\mathcal{T}$. Conditions are given on a semigroup in order that it be a band or semilattice of (1) simple semigroups, (2) completely simple semigroups, and (3) groups. (Received November 12, 1953.)

\section{A. H. Clifford: Naturally totally ordered commutative semi-} groups.

A commutative semigroup $S$ is called naturally totally ordered (n.t.o.) if the division relation in $S$ is a total (i.e. "linear" or "simple") ordering of $S$. O. Hölder [Ber. Verh. Sächs. Akad. Wiss. Leipzig, Math.-Nat. Kl. vol. 53 (1901) pp. 1-64] showed that every archimedean n.t.o. semigroup $S$ in which the cancellation law holds can be embedded in the additive semigroup $P$ of all positive real numbers, and that $P$ is to within isomorphism the only such semigroup which is complete in the sense that every bounded subset of $P$ has a LUB in $P$. In the present paper, analogous results are shown for archimedean n.t.o. semigroups in which the cancellation law does not hold. In this case, there are exactly two non-isomorphic complete such semigroups, in which all others can be embedded. Commutative n.t.o. semigroups in general have been investigated by F. Klein-Barmen [Math. Zeit. vol. 48 (1942-43) pp. 715-734; Math. Zeit. vol. 51 (1947-49) pp. 355-366], who calls them "linear holoids. A reduction theorem is given which generalizes the main result of the first of these two papers. (Received November 12, 1953.)

\section{W. F. Eberlein: A note on Fourier-Stieltjes transforms.}

Given a locally compact Abelian group $G$ and a bounded Radon measure $\mu$ on the character group $G^{*}$, the Fourier-Stieltjes transform $\widehat{\mu}(x)=\int_{G^{*}}(\overline{x, y}) d_{\mu}(y)$ is a weakly almost periodic function on $G$. Then $M\left(|\hat{\mu}|^{2}\right)=\sum a^{*}|\mu\{y\}|^{2}$. Previous results of this type have presupposed the existence of a sequential limit representation for the mean value $M$. (Received November 16,1953.)

188. Gertrude Ehrlich: Structure theorem for continuous rings. Preliminary report.

A regular ring whose lattice of right (or left) ideals is isomorphic to the lattice of subspaces of a continuous geometry is called a continuous ring (see von Neumann, Proc. Nat. Acad. Sci. U.S.A. vol. 22 (1936) pp. 92-100, 101-108 and 707-713; vol. 23 (1937) pp. 16-22 and 341-349). It is shown here that, for a continuous ring which is not of characteristic 2, the multiplicative group of nonsingular elements determines the ring to within isomorphism or anti-isomorphism, and hence determines the associated continuous geometry to within isomorphism or dual isomorphism. This is an 
extension to the continuous case of the structure theorem known for finite- and infinite-dimensional discrete projective geometries. The methods employed are based on Baer's treatment of the discrete case ( $\mathrm{R}$. Baer, Linear algebra and projective geometry, Academic Press, 1952, Chap. VI) in which systems of involutions having a common plus or minus invariant space play a decisive role. (The results presented in this paper constitute part of a doctor's dissertation written at the University of Tennessee under the direction of Professor Wallace Givens). (Received November 4, 1953.)

189. Harley Flanders: The norm function of an algebraic field extension. II.

Let $K$ be a finite extension of a field $k$ and let $F(X)$ be in the ring $K\left[X_{1}, \cdots, X_{n}\right]$. It is shown that if $F$ is irreducible, then $N_{K / k}(F)$ is a power of an irreducible in $k[X]$. From this it follows that the general norm is a prime power; the author's previous result (Pacific Journal of Mathematics vol. 3 (1953) p. 103) characterizing the norm function is a consequence. It is shown that standard properties of the norm and trace follow from this, for example, their transitivity. (Received November 4, 1953.)

190. Franklin Haimo: Semi-direct products with ample homomorphisms. Preliminary report.

Let $G$ be the semi-direct product of groups $H$ and $K$ with a homomorphism $\phi$ on $K$ into the automorphism group of $H$ [A. Malcev, On linear Lie groups, C. R. Acad. Sci. URSS vol. 40 (1943) pp. 87-89]. Let $\phi$ be ample in that $\phi(K) \supset J(H)$, the set of inner automorphisms of $H$. The $G / Z_{i}$ are found, where $Z_{i}(G)=Z_{i}$ is the $i$ th member of the ascending central series of $G$. In particular, a simple formulation is given for the ascending central series of the holomorph of $H$. An equivalent condition is found for each $Z_{i}(G)$ to be the set of all $(h, k), h \in Z_{i}(H), k \in Z_{i}(K)$. This work has been supported, in part, by the OSR, USAF. (Received November 13,1953.)

\section{1t. Marshall Hall and H. J. Ryser: Normal completions of inci- dence matrices.}

Let $A_{1}$ be an $r$ by $v$ incidence matrix of zeros and ones, which satisfies the equation $A_{1} A_{1}^{T}=B_{1}$. Here $A_{1}^{r}$ denotes the transpose of $A_{1}$ and $B_{1}$ is the $r$ by $r$ matrix with $k$ in the main diagonal and $\lambda=k(k-1) /(v-1)$ in all other positions. Let $B$ be the $v$ by $v$ matrix with $k$ in the main diagonal and $\lambda$ in all other positions. Suppose that $B$ is rationally congruent to the identity. Then there exists a $v$ by $v$ rational and normal matrix $A$ having $A_{1}$ as its first $r$ rows such that $A A^{T}=A^{T} A=B$. Integral solutions of the matric equation $A A^{T}=B$ are also studied. In particular, an integral solution of this equation is exhibited for the geometry case of $N=10$. The solution is not normal, and, of course, not a geometry. (Received October 19, 1953.)

\section{Emilie V. Haynsworth: Quasi-stochastic matrices.}

Given a matrix, $A=\left(a_{i j}\right)$, let $R_{i}=\sum_{j=1}^{k} a_{i j}+p \sum_{j-k+1}^{n} a_{i j}(i=1, \cdots, n)$, where $p$ is an arbitrary complex number, and $k$ is an integer, $1 \leqq k \leqq n$. $A$ is here defined as a generalized quasi-stochastic matrix if $R_{i}=S(i=1, \cdots, k)$, and $R_{i}=p S(i=k$ $+1, \cdots, n)$. Obviously, if $A$ has non-negative elements, and $S=p=1, A$ is stochastic. This paper generalizes some of the theorems on stochastic matrices for quasistochastic matrices. For instance, one root is always $S$, and, when $p>0, a_{i j} \geqq 0, S$ is the maximum root. Also, the new bounds for the roots of stochastic matrices obtained by A. Brauer [Duke Math. J. vol. 19 (1952) pp. 75-91] are applied to quasi-sto- 
chastic matrices. In addition to the above generalizations on stochastic matrices, it is shown that certain types of matrices are generalized quasi-stochastic for two different values of $p$, and thus two roots can be found by inspection. Other results concerning the location of the roots of these matrices are given, and group properties for the set are discussed. (Received November 13, 1953.)

\section{Arno Jaeger: On rings of differential operators over fields of} prime number characteristic. II.

Using the definitions and the notations of the first part of the paper (Bull. Amer. Math. Soc. Abstract 59-2-163) the ring $R_{i}$ of differential operators is now shown to be (isomorphic to) the ring of all endomorphisms of $F^{+}$over $F_{i}$. If $A$ denotes the matrix (with elements in $F_{i}$ ) corresponding to the differential operator $\alpha$ relative to the basis $1, x, \cdots, x^{p^{i-1}}$ of $F^{+}$over $F_{i}$ the matrix $A^{*}$ corresponding to the adjoint operator $\alpha^{*}=\sum_{n=0}^{p^{i-1}}(-1)^{n} D^{n} \cdot a_{n}$ is obtained from $A$ by reflection of the elements about the matrix diagonal from the upper right to the lower left. The methods established are applied to find the solutions and to discuss the solvability of differential equations in the usual sense of this theory and of differential equations $\sum_{n=0}^{\infty} a_{n} D^{n} y=0$ of infinite order. (Received December 11, 1953.)

\section{4t. W. E. Jenner: Arithmetics of Lie algebras. Preliminary report.}

Let $\boldsymbol{o}$ be a Dedekind ring of characteristic zero with quotient field $k$ and let $\&$ be a semisimple Lie algebra of finite dimension over $k$. A subring $D$ of $\mathscr{R}$ is said to be integral if it is a finitely-generated 0 -module and contains a $k$-basis for $\mathfrak{R}$. A discriminant ideal $\mathfrak{D}$ is associated with $\mathfrak{D}$ in analogy with the definition for associative algebras but using the discriminant of E. Cartan. If $\mathfrak{O}^{\prime}$ is an integral subring with discriminant $\mathfrak{D}^{\prime}$ and such that $\mathfrak{O}^{\prime} \supseteq \mathfrak{D}$, then $\mathfrak{O}^{\prime} \supset \mathfrak{D}$ if and only if $\mathfrak{D}^{\prime} \supset \mathfrak{D}$. Every integral subring can be embedded in a maximal integral subring. If $\mathfrak{p}$ is a prime of $k$, then $\mathfrak{D}-\mathfrak{p D}$, as an algebra over $\mathfrak{D} / \mathfrak{p}$, is a direct sum of simple nonabelian algebras if $\mathfrak{p}$ does not divide $\mathfrak{D}$. The proof depends on a result of J. Dieudonné. For any prime $\mathfrak{p}$ of $k$ let $\mathfrak{o}_{\mathfrak{p}}$ denote the ring of local integers at $\mathfrak{p}$ and set $\mathfrak{D}_{\mathfrak{p}}=\mathfrak{o}_{\mathfrak{p}} \mathfrak{D}^{\text {. Then }} \mathfrak{D}_{\mathfrak{p}}$ is a multiplicative unit operator on its ideals for almost all $\mathfrak{p}$. A direct-intersection ideal theory is obtained for Lie rings which are multiplicative unit operators on their ideals. (Received November 14, 1953.)

\section{J. B. Kelly: A characteristic property of quadratic residues.}

Let $p$ be a prime of the form $4 k+1$. Let $r_{1}, r_{2}, \cdots, r_{2 k}$ denote the quadratic residues $(\bmod p)$, exclusive of 0 , and let $n_{1}, n_{2}, \cdots, n_{2 b}$ denote the quadratic nonresidues $(\bmod p)$. The following theorem is well known. Theorem I: The set of all residues $r_{i}+n_{j}$ where $i$ is arbitrary but fixed and $j$ runs from 1 to $2 k$ contains exactly as many quadratic residues as nonresidues; so does the set $r_{i}+n_{j}$ where $j$ is arbitrary but fixed and $i$ runs from 1 to $2 k$. We have established the following converse of Theorem I. Theorem II: Let $m$ be a positive integer of the form $4 k+1$. Let the nonzero residues $(\bmod m)$ be divided into two mutually exclusive classes of $2 k$ elements each: $A\left\{1=a_{1}, a_{2}, \cdots, a_{2 k}\right\}$ and $B\left\{b_{1}, b_{2}, \cdots, b_{2 k}\right\}$ such that the set of all residues $a_{i}+b_{j}$, where $i$ is arbitrary but fixed and $j$ runs from 1 to $2 k$, contains exactly as many elements of $A$ as of $B$ and such that the set $a_{i}+b_{j}$, where $j$ is arbitrary but fixed and $i$ runs from 1 to $2 k$, has the same property. Then (1) $m$ is a prime, $p$. (2) $A$ consists of the quadratic residues $(\bmod p)$ and $B$ consists of the quadratic nonresidues $(\bmod p)$. Theorems similar to I and II hold for positive integers of the form $4 k-1$. The analogue of Theorem I is true in any finite field; this is not the case for Theorem 
II. The principal tool employed in the proof of Theorem II is cyclotomy. (Received November 16, 1953.)

\section{6t. M. S. Klamkin: On vector sums and products.}

In most treatments of vectors it is usual to introduce the definitions of vector sums and products ad hoc, and then to develop their properties. In this paper the usual expressions for the vector sum, scalar product, and vector product of two vectors will be derived from postulated properties which have direct physical significance. The vector sum has been derived previously by Poisson in this manner. However, the proof presented here is a modified version. (Received November 14, 1953.)

\section{7t. Serge Lang: A new proof of Harnack's theorem.}

Harnack (Math. Ann. (1876)) proved that there are at most $g+1$ real branches on a real curve of genus $g$. We give a new proof based on the Riemann-Roch theorem. Remark first that if $x$ is a real function on the curve, then $x$ cannot have a single zero of order 1 on a branch $B$. (Indeed, $B$ is homeomorphic to a circle. If $x$ has a single zero $p$ on $B$, then $x$ changes sign in a neighborhood of $p$, and gives a continuous image of $B-\{p\}$. If $x$ has no other zero or pole on $B$, this is impossible.) Now if there are $g+2$ branches, select a place on each branch, and let $p_{1}, \cdots, p_{q+2}$ be these places. Let $q$ be a complex place and its conjugate, so $q$ has degree 2 . The divisor $p_{1}+\cdots+p_{g+2}-(g+1) q$ has degree $-g$. By Riemann-Roch, there exists a function $x$, multiple of this divisor, which will have at most $g$ more real zeros on the branches. Hence on at least two branches $x$ will have a single zero, a contradiction. (Received November 12, 1953.)

\section{8t. B. H. Neumann: Groups covered by permutable subsets.}

Using his results on unitary representations (Duke Math. J. vol. 17 (1950) pp. 437-441), F. I. Mautner has proved (unpublished): If the group $G$ has a finite subgroup $K$ whose double cosets in $G$ permute, and if $H$ denotes the subgroup consisting of all elements with finitely many conjugates in $G$, then $H$ has finite index in $G$. An elementary proof of the sharper result $G=K H$ is now given, and extended as follows: The group $G$ can be covered by permutable boundedly finite subsets if, and only if, $G$ has a subgroup of finite index whose derived group is finite. It is also shown that every finitely generated free group, but no nondenumerable free group, can be covered by permutable finite (but unbounded) subsets. (Received November 13, 1953.)

199t. L. J. Paige: Power associative loop algebras.

Let $L$ be a finite loop, written multiplicatively, and $F$ an arbitrary field. Construct the loop algebra $A$ of $L$ over $F$ by considering the elements of $L$ as linear independent basis elements for a vector space $A$ over $F$ and define multiplication in $A$ by the use of both destributive laws and the definition of multiplication in $L$. If $L$ is not associative (not a group), $A$ will be a linear nonassociative algebra of finite order over $F$. The author proves: (i) For fields $F$ of characteristic not 2, the assumption that $A$ is commutative and power associative implies that $A$ is associative. (ii) The existence of non-commutative, power associative (not associative) loop algebras. (Received November 12, 1953).

200t. M. A. Rosenlicht: Automorphisms of function fields.

This concerns the theorem that the group of automorphisms (leaving the elements 
of the ground field fixed) of an algebraic function field of one variable of genus greater than one over an algebraically closed ground field is finite. An easy proof is given in which one of the classical proofs for function fields over the complex numbers is generalized by replacing integration by the jacobian variety of the function field. The remainder of this paper deals with the extension of this result to the case in which the ground field is not algebraically closed. (Received November 14, 1953.)

\section{1t. E. V. Schenkman: The structure of the group of automorphisms of a nilpotent group.}

Let $G$ be a nilpotent group; that is, let $G^{\omega}=E$, the identity, where $G^{\omega}=\bigcap_{k=1}^{\infty} G^{k}$ and $G^{k}=\left[G^{k-1}, G\right], G^{1}=G$. Let $A$ be the group of automorphisms of $G$ and let $A_{k}$ be the subgroup of automorphisms of $A$ which induce the identity transformation in $G / G^{k}$. Then some of the results obtained are as follows: $A_{2}$ is a normal nilpotent subgroup of $A$ of class at most one less than that of $G ;\left[A_{k}, A_{l}\right] \subseteq A_{k+l-1}$. If $H$ is the holomorph of $G$, then the subgroup of $H$ generated by $G$ and $A_{2}$ is normal in $H$ and nilpotent of the same class as $G$. (Received November 16,1953.)

\section{2t. Alfred Tarski: On equationally complete rings and relation algebras.}

For terminology see Kalicki-Scott, Bull. Amer. Math. Soc. Abstract 58-6-582 and 59-1-116. The following two theorems provide a mathematical characterization for the class of equationally complete rings and for that of equationally complete relation algebras (thus presenting solutions for two problems formulated by Kalicki). Theorem I: A ring $\Re$ with more than one element is equationally complete if and only if, for some prime $p$, either the equations $p x=0$ and $x^{p}=x$, or else the equations $p x=0$ and $x \cdot y=0$, identically hold in $\Re$. Theorem II: A relation algebra $\Re$ (in the sense of Jonsson-Tarski, Amer. J. Math. vol. 74, p. 127 ff.) with more than one element is equationally complete if and only if one of the three equations $0^{\prime} ; 0^{\prime}=0,0^{\prime} ; 0^{\prime}=1^{\prime}$, and $0^{\prime} ; 0^{\prime}=1$, as well as the equation $x ; 1 ; \bar{x} ; 1 ;\left(x \cdot 1^{\prime}+\bar{x} \cdot 0^{\prime}\right) ; 1 ;\left(x \cdot 0^{\prime}+\bar{x} \cdot 1^{\prime}\right)=0$, identically holds in $\Re$. Partial results related to Theorem I are stated in Abstract 59-1-116 [Theorems (4) and (5)]. Also, a result related to Theorem II can be found there [Theorem (9) ]; it is simpler than Theorem II, but applies only to relation algebras in which 1 ' is not included among the fundamental notions. (Received November 4 , 1953.)

\section{Morris Weisfeld: Derivations in division rings. Preliminary re-} port.

The structure of a division ring $\Delta$ with center $\Phi$ is studied in relation to the existence of a restricted $p$-Lie algebra over $\Phi$ of derivations in $\Delta$. If $\mathcal{D}$ is such an algebra, the elements $a$ in $\Delta$ such that $d(a)=0$ for all $d$ in $\mathcal{D}$ form a division subring $\Gamma$ of $\Delta$. It is known that such a situation occurs when $\Delta$ is commutative and is a purely inseparable extension of exponent one of $r$. (Cf. Jacobson, Amer. J. Math. vol. 66 (1944) pp. 645-648.) One can determine conditions for the existence of a Galois correspondence between division subrings of $\Delta$ containing $\Gamma$ and certain Lie subalgebras of $\mathcal{D}$. The ordinary Galois theory does not apply because one can show that all automorphisms of $\Delta$ leaving the elements of $\Gamma$ fixed are inner automorphisms. Some other results concern the extension of a derivation defined in a division subring of $\Delta$ to a derivation in $\Delta$. (Received November 13,1953 .) 


\section{4t. Edwin Weiss: Bounded topological rings.}

A class of topological rings which includes both the compact and the discrete classes is studied without recourse to the structure theory of topological groups. By elementary means, a theorem on semisimple rings, which includes the standard Jacobson theorem and the Kaplansky theorem for the compact case, is proved. The unified proof clarifies the relation between the descending chain condition and compactness. (Received November 14, 1953.)

\section{George Whaples (p) and R. E. MacKenzie: Artin-Schreier} equations in characteristic zero. Preliminary report.

Let $k$ be a regular local field with residue class field of characteristic $p$ and consider the extensions $C / k$ which are cyclic of degree $p$. Known result: If $k$ has characteristic $p$, then every such $C$ is generated by a root of an Artin-Schreier equation $x^{p}-x-\lambda=0,|\lambda|=1$, and there is a simple explicit formula for the norm residue symbol. New result: If $k$ has characteristic 0 , then with certain exceptions every $C$ is generated by a root of an Artin-Schreier equation. A simple formula for the norm residue symbol can at present be defined only for elements of a specially chosen basis for $k$. modulo $N(C / k)$. The exceptional $C$ occur only when $k$ contains primitive $p$ th roots of unity; they are the fields generated by a $p$ th root of a prime element. (Received November 16, 1953.)

\section{ANALYsIS}

\section{6t. R. M. Baer: Some partial ordering relations for self-adjoint} operators,.

Let $A$ and $B$ be self-adjoint and everywhere defined on the hilbert space $H$. Define $A \leqq B$ (strongly) if $E_{B}(\lambda) \leqq E_{A}(\lambda)$, all $\lambda$, where we write $E_{A}(\lambda)$ for the spectral resolution of $A$, and projections are ordered by inclusion of range spaces; define $A \leqq B$ (weakly) if $(A x, x) \leqq(B x, x)$, all vectors $x$ in $H$; and $A \leqq B$ (normally) if $A x$ $\leqq B x$, all $x$ in $H$. Then if $A$ and $B$ are strongly comparable, they are weakly comparable; and strong and weak comparability are equivalent for commuting $A$ and $B$. Consequently, for positive commuting $A$ and $B$, all three forms of comparability are equivalent. All three partial orderings are unitary invariant and induce dualities under the mapping of nonsingular operators to their inverses. The relation between (strong) comparability and algebraic operations over the self-adjoint operator space is studied. (Received November 14, 1953.)

\section{7t. R. M. Baer: Lattice structure in the self-adjoint operator space.}

Let $H$ be a separable hilbert space, and let $R(H)$ be the (real) linear space of all everywhere-defined self-adjoint operators on $H$. We define a quasi-vector lattice to be a real linear space $L$ which is also a lattice with respect to a partial ordering $x \leqq y$, such that if $x \leqq y$ (for $x$ and $y$ in $L$ ), then $x+z \leqq y+z$ for all $z$ in some subspace $Z$ (called the vector lattice center) of $L$. Then, with respect to the strong partial ordering (see preceding abstract) for self-adjoint operators, $R(H)$ is a quasivector lattice with vector lattice center consisting of the scalar operators. $R(H)$ exhibits a number of the lattice characteristics of the abstract $(L)$ and $(M)$ spaces, provided the strong (operator) topology is substituted for the uuiform (norm) topology. The results extend directly to the $R(H)$ intersection with weakly closed operator algebras. (Received November 14, 1953.) 
208. Frederick Bagemihl and Leonard Gillman (p): Generalized dissimilarity of ordered sets.

An ordered set is c-homogeneous if every one of its intervals has power $c=2 \mathrm{No}$. Let $M, N \neq 0$ be ordered sets. $M$ is defined to be pseudo-similar to $N$ if there exists a function $f$, with $f(M)=N$, an ordered set $T$ without gaps, and a set $\left\{M_{t}\right\}_{t \in T}$ of mutually exclusive intervals of $M$, such that $\sum_{t \in T} M_{t}=M$ and $f \mid M_{t}$ is a similarity or anti-similarity transformation of $M_{t}(t \in T) . M$ is defined to be essentially identical to $N$ if there is a pseudo-similarity $f$, with $f(M)=N$, having an associated set of intervals $\left\{M_{t}\right\}_{t \in T}$ such that $f \mid M_{t}$ is the identity on $M_{t}(t \in T)$ minus any border elements that it may have. If an ordered set $M$, with $|M|=\mathfrak{c}$, contains a $\mathrm{c}$-homogeneous subset $D$ that can be imbedded in the continuum, then $M$ is the union of $\mathrm{c}$ mutually exclusive c-homogeneous sets $E_{\sigma}$ having the following properties: There is associated with every $E_{\sigma}$ a certain $u_{\sigma} \in E_{\sigma}$ such that $F_{\sigma}=E_{\sigma}-\left\{u_{\sigma}\right\}$ is a c-homogeneous subset of $D$ dense in $D$. Let $U$ be the set of all these elements $u_{\sigma}$. For any $\tau$, let $I_{\tau}$ be any unbordered interval of $F_{\tau}, A \subset M, B \subset M$, and $|B \cap U|<\mathfrak{c}$. Then if $\left|I_{\tau}-A\right|<\mathfrak{c}$, we have not $A \simeq B^{*}$; if, in addition, $A \cup B \subset D$ and $I_{\tau} \cap A \neq I_{\tau} \cap B$, then $A$ not $\simeq B$; if, furthermore, $A \subset I_{\tau}$ and $A$ is not essentially identical to $B$, then $A$ is not pseudosimilar to $B$. Some extensions, applications, and analogous results are also obtained. (Received November 16, 1953.)

\section{9t. Stefan Bergman: On solutions of linear partial differential equations in three variables.}

Using integral operators (see Trans. Amer. Math. Soc. vol. 68, pp. 468 and 500) which transform functions of two complex variables into harmonic functions $H(x, y, z)$ and solutions $\phi(x, y, z)$ of $\Delta \phi+C \phi=0$, where $C$ is an entire function of $x^{2}+y^{2}+z^{2}$, the author investigates single and multivalued solutions $H$ and $\phi$ and their singularities. He shows that various theorems about harmonic functions of two variables, which one obtains using analytic functions of one complex variable, can be generalized to the case of solutions $\phi$. In particular he determines a complete set of particular solutions $\phi_{n}, n=1,2, \cdots$. This result leads to a new method for solving boundary and eigenvalue problems. Further he shows that between the properties of a subsequence of the coefficients $\left\{A_{m n p}\right\}$ of a series development $\phi=\sum A_{m n p} x^{m} y^{n} z^{p}$ on one side and the location and properties of line singularities of $\phi$ on the other side, there exist simple relations. They are independent of the coefficient $C$ of the equation. They represent a generalization of theorems by Hadamard, Mandelbrojt and others about the relations between the coefficients of a series development of a meromorphic function $f(Z)$ and location of its poles. (Received December 28, 1953.)

\section{0t. F. E. Browder: On eigenfunction expansions for singular ellip-} tic partial differential operators. II. Uniqueness theorems.

By an eigenfunction expansion for an elliptic differential operator $K$ of arbitrary order on a domain $D$ is meant a (possibly infinite) sequence of eigenfunctions $s_{r}(x, \lambda)$ of $(K-\lambda)$ on $D$ and a sequence of Lebesgue-Stieltjes measures $\rho_{r}$ on the real line such that (1) setting $\left(S_{r} f\right)(\lambda)=\int_{D} S_{r}(x, \lambda) f(x) d x, S_{r} f$ is defined in the square mean of $\rho_{r}$; (2) $S_{r}$ is a partially isometric mapping of $L^{2}(D)$ onto $L^{2}\left(\rho_{r}\right)$ isometric on a subspace $H_{r}$; (3) $L^{2}(D)=\sum_{r} \oplus H_{r}$. To every self-adjoint realization $K_{1}$ of a formally self-adjoint elliptic $K$, there corresponds an eigenfunction expansion for which, if $\left\{E_{\lambda}\right\}$ is the resolution of the identity of $K_{1},\left(E_{\lambda}-E_{\mu}\right) f(x)=\sum_{r} \int_{\lambda}^{\mu} s_{r}(x, \lambda)\left\{\int_{D} s_{r}(y, \lambda) f(y) d y\right\} d \rho_{r}(\lambda)$. The correspondence between eigenfunction expansions and self-adjoint realizations 
of $K$ is one-one and both are uniquely determined by the sequence of eigenfunctions $\left\{s_{r}(x, \lambda)\right\}$. The usual uniqueness result for the "limit point" case for ordinary differential operators is a corollary of this general theorem. (Received November 16, 1953.)

\section{R. H. Cameron: The translation pathology of Wiener space.}

In this paper it is shown that almost no translations in Wiener space preserve measurability. It is also shown that Wiener measure is not expressible as an indefinite integral over a $\sigma$-finite translation invariant measure on a $\sigma$-algebra which includes all Wiener measurable sets. Finally, it is shown that an indefinite integral with respect to Wiener measure cannot be translation invariant unless it vanishes identically. (Received October 29, 1953.)

\section{R. C. Carson: Analytic functions in an annulus.}

The following problem is investigated. Let $\mathcal{Z}$ be the family of all functions $f(z)$ regular and single-valued for $q<|z|<1$ which satisfy $|f(z)| \leqq 1,|z|=1$ and $|f(z)|$ $\leqq p,|z|=q$. Given an arbitrary point $z_{0}, q<\left|z_{0}\right|<1$, find a function $F(z)$ in $\mathcal{F}$ such that $\left|F^{\prime}\left(z_{0}\right)\right| \geqq\left|f^{\prime}\left(z_{0}\right)\right|$ for all $f(z)$ in $\mathcal{F}$. The function $F(z)$ is shown to belong to a certain one-parameter subfamily of $\mathcal{F}$ and the function is exhibited for certain classes of values of $p$. The methods used are related to those of R. M. Robinson in Analytic functions in circular rings (Duke Math. J. vol. 10 (1943) pp. 341-353). (Received November 16, 1953.)

\section{3t. Mischa Cotlar: A general interpolation theorem. I.}

Let $T f=T f(x)$ be a linear operator, defined on the set $D$ of step functions of $R^{n}$, such that: (1) $\|T f\|_{2} \leqq C_{2}\|f\|_{2}$ for all $f \in D$. By a theorem of Riesz, (1) and (2) $\|T f\|_{1}$ $\leqq C_{1}\|f\|_{1}$ imply $\|T f\|_{p} \leqq C_{p}\|f\|_{p}(1<p<2)$. Let $S(f)=\{x ;|f(x)|>0\}, m(f)=\inf |f(x)|$ for $x \in S(f), \Lambda f$ a bounded operator on $L^{p} \bigcap D$, and $S_{\Lambda}(f)=\{x ;|\Lambda f(x)| \geqq m(f)\}$. Consider all operators $U f$ on $D$ such that $|U f(x)| \leqq$ cst. $m(f), U f(x)=0$ if $x \in R^{n}-S_{\Lambda}(f)$, and define $\|T f\|_{\Lambda}=\inf _{U} \int_{R^{n}-S_{\Lambda}(f)}|T(f-U f)(x)| d x$. Theorem: Riesz's theorem still holds if one replaces $\|T f\|_{1}$ by $\|T f\|_{\Lambda}\left(\leqq\|T f\|_{1}\right)$ in (2). (Received October 26, 1953.)

\section{4t. Mischa Cotlar: A general interpolation theorem. II.}

For any set $E \subset R^{n}$ define $\|T\|_{\Lambda, E}=\sup \left\{\|T f\|_{\Lambda} /\|f\|_{1}\right\}$ for all $f$ such that $\|f\|_{1} \leqq 1$ and $f(x)=0$ on $R^{n}-E$. For any cube $Q$ define $\|T\|_{\Lambda, Q}=\operatorname{SUP}_{E}\|T\|_{\Lambda, E}$ for $Q / 2 \subset E \subset Q$, and for any $x \in R^{n}$ define $\|T\|_{\Lambda, x}=\inf _{Q}\|T\|_{\Lambda, Q}$ for all cubes $Q$ with center at $x$. Theorem: If, for all $x \in R^{n},\|T\|_{\Lambda, x} \leqq C$, then also $\|T\|_{\Lambda} \leqq C$. For any cube $Q$ let $\mu(f, Q)(x)=$ mean value of $f$ on $Q$ if $x \in Q$ and zero otherwise, and define $\left.\|T f\|_{\mu}=\sup _{Q}\right\rangle_{S(f)} \int_{R^{n}-2 Q} \mid T(f$ $-\mu(f, Q))(x) \mid d x$. Theorem: If $\|T f\|_{\mu} \leqq C_{\mu}\|f\|_{1}$ for all $f \in D$, then $\|T f\|_{\Lambda_{0}} \leqq C_{\Lambda_{0}}\|f\|_{1}$, where $\Lambda_{0} f$ is the Hardy-Littlewood maximal function. (Received October 26, 1953.)

\section{5t. Mischa Cotlar: A general maximal theorem.}

Let $D=\{f(x)\}$ be the set of step functions of $R^{n}, g_{y, \epsilon}(x)$ the characteristic function of the sphere $|x-y|<\epsilon$. For any linear operator $T f=T f(x)$ defined on $D$ define: $\quad T_{\epsilon} f(x)=T f(x)-T\left(g_{x, \epsilon^{\prime}}\right)(x) \quad(\epsilon>0), \quad M T f(x)=\sup _{\epsilon} \quad\left|T_{\epsilon} f(x)\right| . \quad \Delta T f(x)$ $=\sup _{\epsilon>0}\left[\sup _{\left|x-x^{\prime}\right|<\epsilon / 2}\left|T_{\epsilon} f(x)-\left\{T f\left(x^{\prime}\right)-T\left(g_{x, \epsilon} f\right)\left(x^{\prime}\right)\right\}\right|\right]$. (i) If $T f$ and $\Delta T f$ are bounded operators on $D$, considered as a subspace of $L^{p}\left(R^{n}\right)$, then $M T f$ is also bounded on $L^{p} \cap D$. (ii) If $T^{\prime}$ and $T^{\prime \prime}$ are operators in $R^{n}$ and in $R^{m}$ satisfying the hypothesis of Theorem (i), and if $T=T^{\prime} \times T^{\prime \prime}$ is the product operator in $R^{n} \times R^{m}$, then $M T$ is bounded on $L^{p}\left(R^{n} \times R^{m}\right)$. (Received October 26,1953 .) 


\section{6t. Mischa Cotlar: On general Hilbert transforms.}

Let $R^{2}=\{z\}, z=|z| e^{i t}, k(z)=w(t)|z|^{-2}, w(t)$ a smooth function with $\int_{0}^{2 \pi} w(t) d t=0$, $k_{\epsilon}(z)=k(z)$ if $|z|>\epsilon$ and zero otherwise, and $T_{\epsilon} f=f * k_{\epsilon}$ ( $*$ convolution). In a recent paper (Acta Math. vol. 88) Zygmund and Calderon prove the pointwise and mean convergence of $T_{\epsilon} f$ and the "boundedness" of the limit operator $T f$. Similar results hold in $R^{n}$; for $n=1$ this reduces to classical theorems on the ordinary Hilbert transform. These results can be easily derived using the general maximal and interpolation theorems given in the above abstracts. Moreover, the author's methods give more complete results and the generalization for double transforms. In fact, the operator $T f=f * k$ is well defined for any step function $f$ and has the properties: $\Delta T f(x)$ $\leqq$ cst. $\Lambda_{0} f(x)=$ Hardy-Littlewood's maximal function, and $\|T f\|_{\mu} \leqq C_{\mu}\|f\|_{1}$. (Received October 26, 1953.)

217. Frederic Cunningham, Jr.: $L^{1}$-structure in Banach spaces. Preliminary report.

An $L^{p}$-projection in a Banach space is a linear idempotent operator $E$ satisfying the identity $\|E x\|^{p}+\|x-E x\|^{p}=\|x\|^{p}$. Any commutative family of $L^{p}$-projections can be enlarged to a complete Boolean algebra. In particular the family $\varepsilon_{X}$ of all $L^{1}$-projections in $X$ is such an algebra. This leads by methods well known in Hilbert space to the following: a Banach space $X$ is isometric isomorphic to an $L^{1}$ if and only if for all $x \in X$ the set $\left\{E x ; E \in \mathcal{E}_{X}\right\}$ spans a subspace dense in the smallest $L^{1}$-subspace (range of an $L^{1}$-projection) containing $x$; in any case $X$ has an essentially unique representation as an $L^{1}$ continuous direct sum on the Stone space of $\mathcal{E}_{X}$. For any abstract $\sigma$-ring $\mathcal{E}$ let $\mathcal{L}(\mathcal{E})$ denote the space of bounded $\sigma$-additive functions on $\mathcal{E}$ (a notion equivalent to $L^{1}$ ). Then there always exists a unique $\sigma$-ring $\mathcal{E}_{* \text { having a }}$ proper $\sigma$-finite measure and such that $X=\mathcal{L}(\varepsilon)$ and $\mathcal{L}\left(\varepsilon_{*}\right)$ are identical, namely the $\sigma$-ideal in $\varepsilon_{X}$ of those elements whose corresponding principal ideals are measure algebras. The method leads also to a description of all isometric automorphisms of $L^{1}$. (Received November 14, 1953.)

\section{Chandler Davis: Theorems on projections in Hilbert space.}

Here is the central theorem: Let the operators $A(\lambda), 0 \leqq \lambda \leqq 1^{+}$, on the Hilbert space $\mathcal{E}$, satisfy (a) $A(0)=0, A\left(1^{+}\right)=1$; (b) for $\lambda \leqq \mu, A(\lambda) \leqq A(\mu)$; (c) $A(\lambda)=A\left(\lambda^{-}\right)$. Then one constructs a Hilbert space $\mathfrak{H C}$ in which $\bar{\varepsilon}$ is imbedded, and a resolution of the identity $E(\lambda)$ in $\mathcal{H C}$ (for $0 \leqq \lambda \leqq 1^{+}$; normalized $E(\lambda)=E\left(\lambda^{-}\right)$) such that $A(\lambda)$ $=E E(\lambda) E$ (where $E$ is the projection on $\mathcal{E}$ ). In a special case (roughly: no continuous spectra), this follows easily from a construction of Hadwiger; but the method used in the present paper is new. It is related to ideas of Dixmier and others in the case where $E(\lambda)$ is replaced by a single projection. (Received November 16, 1953.)

219t. R. B. Davis: The normal derivative problem for $\partial(\Delta u) / \partial x=0$. Preliminary report.

A boundary value problem existence theorem is proved for the normal derivative problem for $\partial(\Delta u) / \partial x=0$. The regions considered are of rather special type, bounded in part by a straight line. The present paper differs from the writer's earlier work on the normal derivative problem in the following way: free boundary problems, or else problems requiring analytic data, which entered into previous methods of proof, have been eliminated. These have been replaced by a Dirichlet problem for $\Delta u=0$, with a consequent alteration of hypothesis, both as regards prescribed boundary values, and 
also regarding regions considered. Symmetry is again used to reduce to a problem of Sjöstrand. Uniqueness is proved, as well as existence. (Received November 14, 1953.)

\section{Nelson Dunford (p) and Jacob Schwartz: Finitely additive Lebesgue integral.}

Let $\mu$ denote an additive (but not necessarily bounded, finite, or countably additive) function defined on a field of subsets of a set $S$. The theory of measure and integration with respect to $\mu$ can be carried out with suitable modifications, so as to yield many of the standard principal theorems of measure theory. The $\mu$-measurable functions $f(s)$ form an algebra closed with respect to limits in $\mu$-measure. The standard Lebesgue spaces $L_{p}$ can be defined, and it can be shown that convergence in measure plus uniform integrability is equivalent to convergence in norm. This gives a form of the dominated convergence theorem. Consequently, the case of a countably additive measure $\mu$ is distinguished by two principal facts: (i) convergence almost everywhere implies convergence in measure, and (ii) $L_{p}$ is complete. (Received November 13, 1953.)

\section{1t. Albert Edrei: On the zeros of successive derivatives.}

The following analogue of a classical theorem of Landau seems to be new. Let $R(>0)$ be the radius of convergence of the series $1+2+z^{2} / 2+\cdots=f(z)$ and assume $\alpha \neq 1$. Then there exists an absolute constant $C$ such that $R \leqq R_{1}$ $=\max \{C,[C /|\alpha-1|] \log (1 /|\alpha-1|)\}$, or else $f(z) f^{\prime}(z) f^{\prime \prime}(z)$ vanishes at some point of the disk $|z| \leqq R_{1}$. This theorem easily yields some results of Pólya and Saxer [Math. Zeit. vol. 17, pp. 206-227] and also a number of new propositions relative to the limitpoints of zeros of successive derivatives. (Received November 16, 1953.)

\section{Joanne Elliott: Expansion theorems for singular differential operators.}

This paper contains theorems connected with pairs of adjoint operators typified by (1) $d^{2} / d x^{2}+b(x) d / d x$ and (2) $(d / d x)(d / d x-b(x))$ in $-\infty \leqq r_{1} \leqq x \leqq r_{2} \leqq \infty$. Here $b(x)$ is assumed continuous, but not necessarily bounded, in $\left(r_{1}, r_{2}\right)$. Define $B(x)=\int_{x_{0}}^{x} b(s) d s$. If at least one of the integrals $\int_{x_{0}}^{x} e^{B(s)} d s \int_{x_{0}}^{s} e^{-B(t)} d t$ or $\int_{x_{0}}^{x} e^{-B(s)} d s \int_{x_{0}}^{s} e^{B(t)} d t$ converges as $x \rightarrow r_{j}$, then it is shown that, under certain boundary conditions, the eigenfunctions of (1) form a set which is orthonormal with respect to $e^{B}$ and which is complete both in the weighted $L_{2}$ space and in $C\left[r_{1}, r_{2}\right]$. The eigenfunctions $\left\{\phi_{n}\right\}$ and the eigenvalues $\left\{\lambda_{n}\right\}$ are common to both spaces. It is shown that any $f \in C\left[r_{1}, r_{2}\right]$ can be expanded as follows: $\sum_{n=0}^{\infty} e^{\lambda_{n} t} \phi_{n}(x) \int_{r_{1}}^{r_{2}} \phi_{n}(s) f(s) e^{B(s)} d s \rightarrow f(x)$ as $t \rightarrow 0$ in the sense of bounded pointwise convergence. The subspace of $C\left[r_{1}, r_{2}\right]$ on which the convergence is uniform is specified. A similar expansion is proved for (2) on the space $L\left[r_{1}, r_{2}\right]$, but the limit as $t \rightarrow 0$ is a limit in the mean of $L$. The proofs employ primarily the theory of semigroups and the theory of integral equations in a Hilbert space. (Received November 14, 1953.)

223t. Paul Erdös and Michael Golomb: Functions which are symmetric about several points.

Real functions $f(t)$ are constructed that are bounded, not equivalent to a constant, and satisfy the equation $f(x+t)+f(x-t)-2 f(t)=0$ for all $t$ and a nonmeasurable set of $x$ 's that is everywhere dense and of power $c$. This solves a problem proposed by R. P. Boas. It is also shown that there is a bounded complex-valued function $f(u)$, 
not equivalent to a constant, which satisfies the equation $(*) \sum_{k=1}^{n} f\left(z+c_{k} u\right)-f(z)=0$ for all complex $u$ and a set of $z$ 's that is everywhere dense and of power $c$ provided $\sum_{k=1}^{n} \gamma_{k}\left(c_{k} /\left|c_{k}\right|\right)^{p}\left|c_{k}\right| i q=0$ for some real $p, q$. This latter condition is also proved to be necessary for the existence of an essentially bounded $f(u)$ not equivalent to 0 that satisfies $(*)$ for almost all $u$ and at least one number $z$. (Received November 18, 1953.)

224. Michael Fekete: Generalized transfinite diameter and minimum of the energy integral.

Call $g(r)$ a generator function if (a) $g(r)$ is continuous for $0<r<\infty$; (b) $g(r)$ is strictly decreasing with $r^{-1}$; (c) $\lim g(r)=\infty$ for $r \downarrow 0$. Let $S$ be any compact point set of a Euclidean space $E_{q}$ of $q$ dimensions, $q \geqq 1$, finite. Let $\mu(e)$ be a Borel measure on $E_{q}$ such that $\mu(S)=1$. Then the Stieltjes-Radon integrals $u(P)=\int_{S} g(P Q) \mu\left(e_{Q}\right)$ $=\lim _{N \rightarrow \infty} \int_{S} g_{N}(P Q) \mu\left(e_{Q}\right), I(\mu)=\iint_{S} g(P Q) \mu\left(e_{P}\right) \mu\left(e_{Q}\right)=\lim _{N \rightarrow \infty} \iint_{S} g_{N}(P Q) \mu\left(e_{P}\right) \mu\left(e_{Q}\right)$ respectively yield the potential at $P \in E_{q}$ and the energy integral of the unit mass distributed on $S$ in accordance with $\mu(e)$ when the "law of attraction (repulsion)" is given by $g(r)$; here $g_{N}(r)=g(r)$ as long as $g(r)<N$ while $g_{N}(r) \equiv N$ whenever $g(r) \geqq N$; furthermore $e_{P}, e_{Q}$ denote vicinities of $P$ and $Q$ in $S$. These concepts of Frostman (Thesis, 1935, Lund) and the generalized transfinite diameter $\delta=\delta(S, g)$ of $S$ with respect to $g(r)$ (cf. Fekete, Bull. Amer. Math. Soc. Abstract 53-7-288) have the following connections: $I(\mu)$ has a finite minimum if and only if $\delta>0$, being attained for the "equilibrium distribution" $\mu^{*}(e)=\lim _{n \rightarrow \infty} \mu_{n}(e)$ and equal to $g(\delta)$; the corresponding potential: $u^{*}(P)=\lim _{n \rightarrow \infty} n^{-1} \sum_{y=1}^{n} g\left(P P_{y}^{(n)}\right)$ whenever $P \in T$, the complement of $S$ with respect to $E_{q}$. Here $P_{\nu=1}^{n}, 1 \leqq \nu \leqq n$, means any extremal system of order $n \geqq 2$ of $S$ with respect to $g(r)$, minimizing $\sum_{1 \leqq a<b \leqq .2} g\left(P_{a} P_{b}\right)$ when $P_{1}, \cdots, P_{n}$ range over $S$, while $\mu_{n}(e)=n^{-1} \times$ the number of all points $P_{\nu}^{(n)}$ contained in $e$. (Received November 20, 1953.)

\section{F. A. Ficken: Periodic solutions of a nonlinear wave equation.}

Let $L u \equiv u_{x x}-u_{t t}-2 \kappa u_{t}-\alpha u$, with $\kappa$ and $\alpha$ positive constants. Let $b(x, t)$ be such that $b(0, t)=0=b(\pi, t)$ and $b(x, t+p)=b(x, t)$. The initial value problem $L u=b$ $+\epsilon u^{3}$ ( $\epsilon$ constant), $u(0, t)=0=u(\pi, t), u(x, 0)=f(x), u_{t}(x, 0)=g(x)$ was shown by B. A. Fleishman (Bull. Amer. Math. Soc. Abstract 59-4-365) to have a solution $u(f, g ; x, t)$. He also proved that $f$ and $g$ can be so chosen that $u$ is $p$-periodic in $t$ : $u(f, g ; x, t+p)=u(f, g ; x, t)$ under certain conditions including requirements that $\left|\alpha-\kappa^{2}\right|$ and $\epsilon$ be small. Similar results are established here for $\kappa>0, \alpha>0$, and sufficiently small $\epsilon$ without restriction on $\left|\alpha-\kappa^{2}\right|$. Instead of an integral operator inverting $u_{x x}-u_{t \iota}$, we use here an integral operator $M$ inverting $L u ; M$ is obtained by solving a nonhomogeneous telegraph equation. The solution for $f(x)$ and $g(x)$ of the functional equations $u(f, g ; x, p)=f(x), u_{t}(f, g ; x, p)=g(x)$ expressing the periodicity conditions is also simplified in some respects. (Received November 12,1953.)

\section{6t. Y. Fourès and I. E. Segal: Causality and analyticity.}

A real vector group $G$ is causally oriented if there is distinguished in $G$ a proper convex cone with interior. An operator in a space of functions on $G$ is homogeneous if it commutes with translations by elements of $G$, and a homogeneous operator is causal if it maps those elements of its domain that vanish outside $C$ into functions vanishing outside $C$. A closed homogeneous densely defined operator $T$ on the space $L_{2}(G, K)$ of square-integrable functions on $G$ with values in the complex Hilbert space $K$ is characterized by its gain, which is the measurable function on the dual $G^{*}$ of $G$ to the closed operators on $K$ multiplication by which, acting on $L_{2}\left(G^{*}, K\right)$, is the Plan- 
cherel transform of $T$. Various results are obtained essentially to the effect that $T$ is causal if and only if its gain admits an analytic extension to the tube over the dual of $C$ which does not grow too rapidly, a simple necessary and sufficient conditions for causality being obtained for bounded operators and for Green's operators of partial differential equations with constant coefficients. (Received December 2, 1953.)

\section{7t. E. E. Floyd and V. L. Klee: A characterization of reflexivity} by the lattice of closed subspaces.

Šmulian proved that a closed convex subset of a normed linear space fails to be weakly compact if and only if it contains a decreasing sequence of closed convex sets whose intersection is empty. The authors establish here a similar result: $a$ bounded closed convex subset $C$ of a normed linear space fails to be weakly compact if and only if there is a decreasing sequence of closed linear manifolds whose intersection is empty and each of which intersects $C$. Applied to the unit cell of the space, this yields a new characterization of reflexivity, and from it follows the principal result of the paper: $A$ normed linear space is reflexive if and only if the lattice of all its closed linear subspaces is, in the order topology of G. Birkhoff, a Hausdorff space. (Contrast this with Theorem 13 on page 60 of Lattice theory.) (Received October 30,1953.)

\section{A. W. Goodman: Almost bounded functions.}

Let $G=\left\{L_{1}(w), L_{2}(w), \cdots, L_{2 n}(w)\right\}$ be a group of linear transformations of even order. A function $f(z)$ is said to be almost bounded with respect to the group $G$ in a region $R$ if it is meromorphic in $R$ and if for each $w, f(z)$ assumes in $R$ at most $n$ values from the set $\left\{L_{1}(w), L_{2}(w), \cdots, L_{2 n}(w)\right\}$. The simple case $G=\{w, 1 / w\}$ has been studied extensively. In this paper all of the groups of linear transformations of finite order are examined, and certain standard forms are selected to represent the equivalence class. Let $\&$ denote any group in its standard form. It is proved that if $f(z)=a_{1} z+a_{2} z^{2}+\cdots$ is univalent and almost bounded for $\$ 5$ in $|z|<1$, then $\left|a_{1}\right| \leqq 1$ with equality if and only if $f(z)=\eta z,|\eta|=1$. (Received November 14,1953 .)

\section{9t. W. T. Guy, Jr.: Jacobian extensors and fractional differentia-} tion.

The concept of Jacobian extensor is extended from integral order to nonintegral order by use of one of the fractional differentiation operators. A contrast is made with the generalization due to Hiroyoshi Sasayama. (Received November 16, 1953.)

\section{Violet B. Haas: Singular perturbations of solutions of a non-} linear differential equation.

The differential equation, $\epsilon \ddot{x}+f(t, x, \dot{x} ; \epsilon)=0$ is considered, where $\epsilon$ is a small positive parameter. $f(t, x, y ; \epsilon)$ belongs to class $C^{2}(t, x, y)$ in the domain $D:\left[T_{0} \leqq t<\infty\right.$, $|x| \leqq X,|y|<\infty], f$ and its partial derivatives being bounded here. $f_{y y}$ is not identically zero in $D$, and $f$ is continuous in $\epsilon$ for $0 \leqq \epsilon \leqq \epsilon_{0}$. Let $x=x(t)$ be a solution of the differential equation under discussion, and let $z=z(t)$ be a solution of the corresponding degenerate equation $(\epsilon=0)$. Let $t_{0}>T_{0}$, and let $\left|x\left(t_{0}\right)-z\left(t_{0}\right)\right| \leqq \delta_{1},\left|\dot{x}\left(t_{0}\right)-\dot{z}\left(t_{0}\right)\right|$ $\leqq \delta_{2} / \epsilon$. If $t_{1}$ and $q$ are such that $f\left(t_{1}, z\left(t_{1}\right), q ; 0\right)=0, f_{y}\left(t_{1}, z\left(t_{1}\right), q ; 0\right)=0, t_{1}>t_{0}$, then under certain added conditions if $\tau_{1}, \tau_{2}$ are any two numbers satisfying $t_{0}<\tau_{1}<\tau_{2}<t_{1}, \mid x(t)$ $-z(t) \mid \rightarrow 0$ uniformly for $t_{0} \leqq t \leqq \tau_{2}$ and $|\dot{x}(t)-\dot{z}(t)| \rightarrow 0$ uniformly for $\tau_{1} \leqq t \leqq \tau_{2}$ as $\epsilon$, $\delta_{1}, \delta_{2} \rightarrow 0$. Furthermore, $x(t)$ has rapid oscillatory behavior for $t>t_{1}$, the amplitude and period of these oscillations being not constant for fixed $\epsilon$ and approaching $\left|z\left(t_{1}\right)\right|$ 
and $O\left(\epsilon^{1 / 2}\right)$ respectively as $\epsilon \rightarrow 0$. The proof depends partially on some results of $\mathrm{N}$. Levinson [Acta Math. vol. 82 (1950) pp. 71-106] and partially on certain qualitative estimates on the behavior of solutions in phase space. (Received November 14,1953.)

\section{1t. M. H. Heins: The set of asymptotic values of an entire function.}

The set of asymptotic values of a meromorphic function is an analytic set (Mazurkiewicz, Kierst). An example of a meromorphic function in the unit circle whose set of asymptotic values is an assigned analytic set of the extended plane has been constructed by Kierst. The problem arises to characterize the set of asymptotic values of a nonconstant entire function. It is shown that a set of the extended plane is the set of asymptotic values of a nonconstant entire function if and only if it is analytic and contains the point at infinity. The proof is based upon the use of the representation theorem of Lusin and Sierpinski (as in the work of Kierst) and a direct geometric construction of an entire function which has the desired properties. (Received October 19, 1953.)

\section{2t. M. H. Heins: Universal Blaschke products.}

Let $B$ denote the class of functions which are analytic and of modulus at most one in $|z|<1$. An example is constructed of a convergent infinite Blaschke product $b$ such that (1) for each $f \in B$ there exists a monotone increasing sequence of positive numbers $\left\{x_{n}\right\}_{0}^{\infty}$ with $\lim x_{n}=1$ such that the sequence $\left\{b\left(\left(z+z_{n}\right) /\left(1+x_{n} z\right)\right)\right\}$ tends uniformly to $f$ in $|z|<1$, (2) the zeros of $b$ cluster only at $z=1$. The proof is based upon the fact that there exists a sequence $\left\{b_{n}\right\}_{0}^{\infty}$ of finite Blaschke products which satisfy: (1) $b_{n}(-1)=b_{n}(1)=1$ for $n=0,1, \cdots,(2)$ for each $f \in B$ there exists a subsequence $\left\{b_{n(k)}\right\}_{0}^{\infty}$ which tends uniformly to $f$ in $|z|<1$. By this example an affirmative answer is given to the following question proposed to the author by Professor J. L. Walsh: Does there exist a bounded analytic function $g$ in $|z|<1$ such that for each $f \in B$ there exists a sequence $\left\{T_{n}\right\}$ of non-euclidean motions with the property that $\left\{g \circ T_{n}\right\}$ tends uniformly to $f$ in $|z|<1$ ? With the aid of the indicated example one can construct a convergent infinite Blaschke product $b^{*}$ such that given $f \in \mathcal{B}$ and $\zeta$ on the unit circumference there exists a sequence of non-euclidean motions $\left\{T_{n}\right\}$ with $\lim T_{n}=\zeta$ and $\lim b^{*} \circ T_{n}=f$. (Received October 19,1953.)

\section{Einar Hille and George Klein (p): On Riemann's localization theorem for Fourier series.}

Let $S_{n}(x ; f)$ be the $n$th partial sum of the Fourier series of the $L$-integrable, nonconstant function $f(x)$ of period $2 \pi$, and let $R$ be the difference $R(x, \delta, n, f)=S_{n}(x ; f)$ $-\pi^{-1} \int_{-\delta}^{\delta} f(x+t) t^{-1} \sin n t d t$. The theorem of the title states that for each $\delta, 0<\delta<\pi$, $R \rightarrow 0$ uniformly in $x$ as $n \rightarrow+\infty$. This result is sharpened by establishing that $|R|$ $\leqq A \delta^{-1}\left(\|f\|_{1}+1\right) \omega_{1}\left(n^{-1} ; f\right)$, where $A$ is an absolute constant, $\|f\|_{1}=(2 \pi)^{-1} \int_{-\pi}^{\pi}|f(x)| d x$, and $\omega_{1}(h ; f)=\sup _{|t| \leqq}|h|\|f(x+t)-f(x)\|_{1}$. A result of independent interest obtained in the course of the proof is that for all $x, \int_{0}^{h}|f(x+t)| d t \leqq A\left(\|f\|_{1}+1\right) \omega_{1}(h ; f)$. (Received November 10, 1953.)

\section{I. I. Hirschman, Jr.: On a conjecture of Hewitt.}

Let $G$ with elements $x, y, \cdots$ be a locally compact Abelian group, and let $G^{\widehat{ }}$ with elements $x \widehat{x}, \widehat{y}, \cdots$ be its dual. Haar measure in $G$ is denoted by $d x$, and in $G^{\widehat{ }}$ by $d x^{\widehat{ }}$. We suppose that these measures are so adjusted that if $\hat{f}\left(x^{\wedge}\right)$

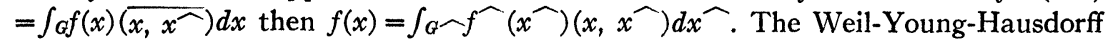


theorem asserts that if $f(x) \in L^{p}(G), 1 \leqq p \leqq 2$, and if $p^{-1} q^{-1}=1$ then $\left\|\widehat{f^{-}}(\widehat{x})\right\|_{q}$ $\leqq\|f(x)\|_{p}$. The cases of equality for $1<p<2$ have been investigated by E. Hewitt,

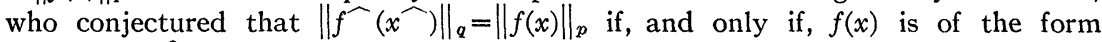
$c \chi_{y A}(x)(x, \widehat{y})$, where $c$ is a complex constant, $y$ an element of $G, A$ an open and compact subgroup of $G, \chi_{y A}(x)$ the characteristic function of $y A$, and $y$ an element of $G^{-}$. Hewitt verified this result in the cases when $1<p<4 / 3$, and when $G$ is compact or discrete. The present paper is devoted to establishing this conjecture without restriction. The method employed depends upon an examination of the conditions for equality in the Riesz-Thorin convexity theorem. (Received November 9, 1953.)

\section{5t. A. J. Hoffman: On an inequality of Hardy, Littlewood, and Pólya.}

A well known inequality of Hardy, Littlewood, and Pólya (Inequalities, p. 49) is strengthened as follows: let $\alpha_{1} \geqq \alpha_{2} \geqq \cdots \geqq \alpha_{n}$ be given. Then in order that $\beta_{1}, \beta_{2}, \cdots, \beta_{n}$ satisfy $\beta_{1} \geqq \beta_{2} \geqq \cdots \geqq \beta_{n}$ and $\beta_{1} \leqq \alpha_{1}, \cdots, \beta_{1}+\cdots+\beta_{n-1} \leqq \alpha_{1}+\cdots$ $+\alpha_{n-1}, \beta_{1}+\cdots+\beta_{n}=\alpha_{1}+\cdots+\alpha_{n}$, it is necessary and sufficient that there exist $n^{2}$ numbers $a_{i j}$ such that $\beta_{i}=\sum a_{i j} \alpha_{j}$, where the matrix $\left(a_{i j}\right)$ is stochastic, symmetric, and satisfies $i \leqq j, i^{\prime} \leqq j^{\prime}, i \geqq i^{\prime}, j \leqq j^{\prime} \rightarrow a_{i j} \geqq a_{i^{\prime} j^{\prime}}$. The proof follows readily from an examination of the graphs of $A_{t}=\sum_{i=1}^{t} \alpha_{i}$, and $B_{t}=\sum_{i=1}^{t} \beta_{i}$. (This work was supported (in part) by the Office of Scientific Research, USAF.) (Received November 14, 1953.)

236t. A. J. Hoffman: On the singular values of the product of two operators.

Let $A, B, C$ be $n \times n$ matrices with singular values $\alpha_{1} \geqq \cdots \geqq \alpha_{n}, \beta_{1} \geqq \cdots \geqq \beta_{n}$, $\gamma_{1} \geqq \cdots \geqq \gamma_{n}$ respectively, and let $C=A B$. Let $0<i_{1}<i_{2}<\cdots<i_{m} \leqq n$. Then $\gamma_{i_{1}} \gamma_{i_{2}} \cdots \gamma_{i_{m}} \alpha_{i_{1}} \alpha_{i_{2}} \cdots \alpha_{i_{m}} \beta_{1} \beta_{2} \cdots \beta_{m}$. This result is equivalent to a theorem of Lidskiy on the eigenvalues of the product of two positive definite matrices. The new proof, a modification of ideas used by $\mathrm{H}$. Wielandt in proving a similar theorem for eigenvalues of the sum of Hermitian matrices, is based on a minimax characterization of the product $\lambda_{i_{1}} \lambda_{i_{2}} \cdots \lambda_{i_{m}}$, where $\lambda_{i} \geqq \cdots \lambda_{n} \geqq 0$ are the eigenvalues of a Hermitian positive semi-definite matrix. The main theorem can also be shown to hold if $A, B, C$ are completely continuous operators in Hilbert space. (This work was supported (in part) by the Office of Scientific Research, USAF.) (Received November $14,1953$.

237t. J. C. Holladay: On conjugacy of some transformations of the interval.

Two continuous transformations $f(x)$ and $g(x)$ mapping the interval $0 \leqq x \leqq 1$ into itself are called conjugate if there exists a homeomorphism, $h(x)$, of this interval such that $f(x)=h g h^{-1}(x)$. A result of S. Ulam and J. von Neumann (On combination of stochastic and deterministic processes, Bull. Amer. Math. Soc. Abstract 53-11-403) establishes the conjugacy of the functions $f(x)=4 x(1-x)$ and $g(x)=2 x$ for $0 \leqq x \leqq 1 / 2$ and $g(x)=2-2 x$ for $1 / 2 \leqq x \leqq 1$. A general study of this notion is undertaken for certain types of transformations. Necessary and sufficient conditions are given for conjugacy between more general transformations. This study involves the investigation of measures, invariant under the inverses of the transformations, and of their sometimes ergodic and mixing behavior. One of the results is that for a transformation of a certain type, including the above-mentioned transformation, there exists a non-denumerably infinite number of linearly independent measures of the interval, 
assigning positive values to each subinterval, for which the transformation is mixing. (Received November 9, 1953.)

238t. M. A. Hyman: The order of the remainder term in Taylor expansions.

A well known corollary of Taylor's Theorem is "Given that $F(x), F^{\prime}, F^{\prime \prime}, \cdots$, $F^{(n)}$ exist and are continuous at all points of an interval $I$, then for two points $x_{0}, x_{0}+h$ in $I, F\left(x_{0}+h\right)=F\left(x_{0}\right)+h F^{\prime}\left(x_{0}\right)+\left(h^{2} / 2\right) F^{\prime \prime}\left(x_{0}\right)+\cdots+\left(h^{n} / n !\right) F^{(n)}\left(x_{0}\right)+o\left(h^{n}\right)$." The small $o$ has its usual order-of-magnitude meaning. However, the assumed continuity of the last derivative can be dropped; the relationship holds in the neighborhood of any point $x_{0}$ for which $F^{(n)}\left(x_{0}\right)$ exists. Without difficulty this result can be extended to more general forms of Taylor's Theorem, in particular to cases involving more than one independent variable. (Received November 3,1953.)

\section{J. R. Isbell: Pathology of monotone set functions.}

Let $f$ be a monotone real function, continuous in the order topology, on a measure algebra which is either complete as a Boolean algebra or complete as a measure algebra; the range of $f$ is compact. However, there exist two such functions, $f_{1}, f_{2}$, such that both the range of $f_{1}-f_{2}$ and the set of all pairs $\left(f_{1}(x), f_{1}(y)\right), x \cap y=0$, are not compact. (Received November 12, 1953.)

240. M. L. Juncosa (p) and D. M. Young: On the Crank-Nicolson procedure for parabolic partial differential equations.

In Proc. Cambridge Philos. Soc. (1947) J. Crank and P. Nicolson proposed replacing $u_{t}$ and $u_{x x}$ by $[u(x, t+k)-u(x, t)] / 2 k$ and $[u(x+h, t+k)+u(x-h, t+k)$ $-2 u(x, t+k)+u(x+h, t)+u(x-h, t)-2 u(x, t)] / 2 h^{2}$ respectively for numerical solution of quasi-linear parabolic partial differential equations. For the case of $u_{t}=u_{x x}$ with homogeneous boundary conditions and fairly general conditions on the initial function $f(x)$, convergence proofs of the Crank-Nicolson procedure are given. Bounds on the order of convergence are also obtained for different classes of the initial function. When $f(x)$ is a step function or of bounded variation, the bounds are $O\left(h^{-1}\right)$. When $f(x)$ has a derivative of bounded variation, has a continuous graph composed only of straight-line segments, or is a step function and the mesh points are preserved through the limiting process, the bounds are $O\left(h^{-2}\right)$. If $f(x)$ or its derivative satisfy a Lipschitz condition of order $\alpha \leqq 1$, the bounds are $O\left(h^{-\alpha}\right)$ or $O\left(h^{-1-\alpha}\right)$ respectively. If $f(x)$ has a modulus of continuity $\omega(\delta)$, then the bounds are max $\left\{O(\omega(h)), O\left(h^{2}\right)\right\}$ and if $f^{\prime}(x)$ exists and has a modulus of continuity $\omega_{1}(\delta)$, then the bounds are $\max \left\{O(h \omega(h)), O\left(h^{2}\right)\right\}$. Since no better bounds than $O\left(h^{2}\right)$ are given and bilinear interpolations on functions of two variables are good to $O\left(h^{2}\right)$, the results are also valid for extensions of the approximate solutions of the differential equation by bilinear interpolation. (Received December 11, 1953.)

\section{W. B. Jurkat: Some new inclusion theorems for Nörlund means.}

For the inclusion $N\left(p_{n}\right) \subseteq N\left(q_{n}\right)$ sufficient monotony conditions are obtained similar to the well known conditions of Hardy. Let $N\left(q_{n}\right)$ be regular and $p_{0}=q_{0}=1$. Then a typical pair of sufficient conditions is $0\left\langle p_{n} \downarrow, q_{n}-p_{n} \nearrow\right.$, including the special theorem $N\left(p_{n}\right) \subseteq C_{1}$ for decreasing positive $p_{n}$. There are some more general results using the differences of higher order of $p_{n}$ and $q_{n}$. They contain e.g. a theorem for Nörlund means analogous to the familiar second theorem of consistency for Riesz 
means. The proof of these results depends on some theorems on the distribution of signs in a power series formerly obtained. (Received November 14, 1953.)

\section{2t. W. B. Jurkat: The Faltung of Nörlund means.}

A Nörlund method $N\left(p_{n}\right)$ is given by the linear transformation $\sigma_{n}=\left(1 / p_{n}\right)$ $\cdot \sum_{\nu=0}^{n} p_{n-\nu} s_{\nu}$ where $p_{n}$ is real and $P_{n}=\sum_{\nu=0}^{n} p_{\nu} \neq 0$. A method $N\left(\tau_{n}\right)$ is said to be the Faltung of $N\left(p_{n}\right)$ and $N\left(q_{n}\right)$, in signs $N\left(\tau_{n}\right)=N\left(p_{n}\right) * N\left(q_{n}\right)$, if $\tau_{n}=\sum_{\nu=0}^{n} p_{\nu} q_{n-\nu}$. Every inclusion theorem for Nörlund means can be stated in the form $N\left(p_{n}\right) * N\left(q_{n}\right)$ $\supseteq N\left(p_{n}\right)$ or $\supseteq N\left(q_{n}\right)$, and for these inclusions general sufficient conditions are obtained extending the well known theorem of Nörlund. A typical theorem is that both inclusions hold if $N\left(p_{n}\right)$ and $N\left(q_{n}\right)$ are regular and one of the methods is positive, for instance $p_{n} \geqq 0$. As a special result one has $N\left(p_{n}\right) \subseteq M\left(P_{n}\right)$ for all regular methods $N\left(p_{n}\right)$. Furthermore some equivalence theorems are obtained, for instance $N\left(p_{n}\right)$ * $N\left(q_{n}\right)$ is equivalent to the regular method $N\left(q_{n}\right)$ if and only if $N\left(p_{n}\right)$ is equivalent to convergence. (Received December 10, 1953.)

\section{N. D. Kazarinoff: Asymptotic expansions for the Whittaker} functions of large complex order $m$.

The investigation of the Whittaker functions $M_{k, m}(x)$ and $W_{k, m}(x)$ which the writer announced previously (Bull. Amer. Math. Soc. Abstract 59-4-468) has been completed. The dominant terms in the asymptotic expansions of these functions for large $|m|$ have been obtained explicitly. These dominant terms are seen to be simple rational and exponential functions of $x$ except when $|x / 2 m|$ is close to one. When $|x / 2 m|$ is close to or equal to one the behavior of $W_{k, m}(x)$ and $M_{k, m}(x)$ is given in terms of that of the Bessel functions $J_{1 / 3}(\xi)$ and $J_{-1 / 3}(\xi), \xi \sim m\left[(x / 2 i m)^{2}-1\right]^{3 / 2} / 3$. (Received November 12, 1953.)

\section{J. H. B. Kemperman: Generalizations of the Euler-Maclaurin} formula.

Let $Q(x)$ be integrable in $[0,1]$ and $\int_{0}^{1} Q(x) d x=1$. Define the generalized Bernoulli numbers $B_{h}(h=0,1, \cdots)$ recursively by $(B+1)^{m}-B^{m}=m \int_{0}^{1}(1-x)^{m-1}(1-Q(x)) d x$ $\left(m=2,3, \cdots ; B_{0}=1 ; B^{h}\right.$ to be interpreted as $\left.B_{h}\right)$. Let $k ! P_{k}(x)=(x+B)^{k}-k$ $\left.\cdot \int_{0}^{x}(x-y)^{k-1}(1-Q d y)\right) d y(0 \leqq x \leqq 1 ; k=1,2, \cdots)$. Finally, $Q(x+h)=Q(x), P_{k}(x+h)$ $=P_{k}(x)$ for $h=0,1, \cdots$. Let $\Gamma$ be a smooth curve connecting $\xi_{0}$ with $\xi_{n}$ and intersecting $\operatorname{Re}(x)=m$ in exactly one point $\xi_{m}=m+i \eta_{m}(m=0,1, \cdots, n)$. Let $f(x)$ be a function on $\Gamma$ which has an integrable $k$ th derivative. Consider the formula $\sum_{m=1}^{n-1} f_{m}$ $=\int_{\Gamma} Q(x) f(x) d x+\sum_{j=1}^{k}(-)^{i} P_{j}\left(\xi_{n}-0\right) f^{(j-1)}\left(\xi_{n}\right)-\sum_{j=1}^{k}(-)^{i} P_{j}\left(\xi_{0}+0\right) f^{(j-1)}\left(\xi_{0}\right)+R_{k}$, where $f_{m}=\sum_{h=0}^{k-1} f^{(h)}\left(m+i \eta_{m}\right)\left(-i \eta_{m}\right)^{h} / h$ ! and $R_{k}=(-)^{k+1} \int_{\mathrm{r}} f^{(k)}(x) P_{k}(x) d x$. This formula holds: (i) If $Q(x)$ is a trigonometric polynomial, in which case $P_{k}(x)$ is analytic for $0<\operatorname{Re}(x)<1$. (ii) If $\Gamma$ is the interval $[0, n]$ on the real axis, in which case $f_{m}=f(m), P_{j}(n-0)=P_{j}(+0)=B_{j} / j !(j>1), P_{1}(n-0)=1+B_{1}, P_{1}(+0)=B_{1}$. Let $x=x_{1}$ $+i x_{2}$. Suppose that for $0 \leqq x_{1} \leqq n, f(x)$ is analytic except for a finite number of poles and that $f(x)=O\left(e^{2 p \pi\left|x_{2}\right|}\right)$ for large $\left|x_{2}\right|$ with $p<1$. Then, in both cases, $R_{k}=S-I_{0}+I_{n}$, where $S$ is the finite sum of certain residues and $I_{x}=\int_{-\infty}^{\infty} \phi_{k}(v) f^{(k)}(x+i v) d v, \phi_{k}(v)$ only depending upon $Q(x)$. This result generalizes the so-called complex form of the Euler formula. (Received November 14, 1953.)

245. Fulton Koehler: The maximum value of an arbitrary real linear combination of the coefficients of a bounded power series.

Let $f(z)=\sum_{\sigma_{\infty 0}}^{\infty} a_{\sigma} z^{\sigma},|f(z)|<1$ for $|z|<1$. For given real numbers $\lambda_{0}, \lambda_{1}, \cdots, \lambda_{n}$, 
not all zero, it is shown that the maximum of $\left|\sum_{\sigma=0}^{n} \lambda_{\sigma} a_{\sigma}\right|$ is attained when $f(z)$ is of the form $Q_{k}(z) / z^{k} Q_{k}(1 / z)(k \leqq n)$, where $Q_{k}(z)$ is a polynomial of degree $k$ whose zeros all lie in $|z|<1$. The actual value of the maximum is given implicitly from the solution of a system of algebraic equations. Szász [Math. Zeit. vol. 1 (1918) pp. 163183] found an upper bound for $\left|\sum_{\sigma_{m 0}}^{n} \lambda_{\sigma} a_{\sigma}\right|$ for arbitrary complex $\lambda_{\sigma}$, but this upper bound is the actual maximum only in certain special cases, including the cases $\lambda_{o}=1$ and $\lambda_{\sigma}=1-\sigma(n+1)$ which had been solved previously by Landau [Archiv der Math. und Physik (3) vol. 21 (1913) pp. 42-50 and pp. 250-255; vol. 24 (1916) pp. 250-260] and Fejér [Rend. Circ. Mat. Palermo vol. 38 (1914) pp. 79-97] respectively. Other special cases have been solved by Schur and Szegö [Preussischen Akad. WissSitzungsber. (1925) pp. 545-560] and by Rogosinski and Szegö [Math. Zeit. vol. 28 (1928) pp. 73-94]. The idea of the present method is to choose a subset of admissible functions $f(z)$ on which the maximum can be found by elementary methods and then to show that such a maximum holds for all admissible $f(z)$. (Received November 4 , 1953.)

246t. R. E. Lane: A condition necessary and sufficient for a continued fraction expansion of a function to be equal to the function.

Suppose that $f$ is a function and that a continued fraction expansion for $f$ is obtained from a sequence of identities $f=a_{1} /\left(b_{1}-u_{2}\right), u_{p}=a_{p} /\left(b_{p}-u_{p+1}\right)$, where $a_{p-1} \neq 0$, $p=2,3,4, \cdots$ We use the notation of a recent paper (Proc. Amer. Math. Soc. vol. 3 (1952) p. 914); if the continued fraction converges, then there is a sequence $\left\{R_{p}\right\}_{p=1}^{\infty}$ which is in $B_{f}$. In the present paper we show that if $\left\{R_{p}\right\}_{p=1}^{\infty}$ is in $B_{f}$, then for the continued fraction to be equal to $f$ it is necessary and sufficient that there be an infinite sequence $P$ of positive integers such that if $p$ is in $P$ then $u_{p+1}$ is in $t_{p}^{-1}\left(R_{p}\right)$, which is a circle plus its exterior. Moreover, if there is such a sequence $P$ and if $p$ is a positive integer, then the value of $f$ and the $p$ th approximant of the continued fraction are in $R_{p}$. (Received November 13,1953 .)

\section{7t. J. P. LaSalle: Basic principle of the "bang-bang" servo.}

The differential equation of a simple servo system consisting of a power source, a feedback circuit, and a motor can be written in the form $\ddot{x}+g(x, \dot{x})=f(x, \dot{x})$, where $x$ is the error in positioning the motor and $g(x, \dot{x})$ is determined by the motor, and $f(x, \dot{x})$ depends on the feedback circuit. The power available to the feedback circuit is limited and $f(x, \dot{x})$ is limited to values between $\pm F$. In the past it has been assumed on intuitive grounds (see: D. McDonald Nonlinear techniques for improving servo performance, Cook Research Laboratories Bulletin S-2, Chicago, 1950, and D. W. Bushaw, Ph.D. Thesis, Department of Mathematics, Princeton University, 1952) that errors can be reduced to zero in the shortest time by always using the full strength of the power source to apply full acceleration or deceleration (i.e., $f$ is restricted to the values $\pm F$ and such a system is called a "bang-bang" servo). This assumption is given conditional justification. If for a given function $g$, there is a unique "bang-bang" system which is the best of all "bang-bang" systems, then it is shown that it is the best of all possible systems operated from the same power source. In a number of cases Bushaw (loc. cit.) has shown the existence of such unique "bangbang" systems and in these cases the "bang-bang" system is therefore shown to be the best of all possible systems. (Received November 16, 1953.)

248. R. B. Leipnik: Embedding a semi-group of linear operators into a group. Preliminary report. 
If $\left\{P^{a}, a>0\right\}$ is a semi-group of linear operators and $A$ is a linear operator such that $A P^{a+1}=P^{a}$ for $a>0$, sufficient conditions are derived for the existence of a group $\left\{Q^{a},-\infty<a<\infty\right\}$ of operators such that $Q^{a}=P^{a}$ for $a>0, Q^{0}=I$ the identity operator, and $Q^{-n}=A^{n}$ for $n=1,2, \cdots$. The most interesting condition is that for each $a>0$, there is an integer $n_{a}$ such that $A^{n}\left(P^{a} A-A P^{a}\right)=0$ for $n \geqq n_{a}$. The abstract analogue of this weak kind of commutativity has curious properties in an associative ring. (Received December 14, 1953.)

\section{J. J. Levin: First order partial differential equations containing a small parameter.}

The behavior, as $\epsilon \rightarrow 0+$, of the solution of (1) $\epsilon\left[a_{1}\left(x_{1}, x_{2}, u\right) \partial u / \partial x_{1}\right.$ $\left.+a_{2}\left(x_{1}, x_{2}, u\right) \partial u / \partial x_{2}\right]=b\left(x_{1}, x_{2}, u\right)$ passing through a fixed initial curve, $C$, is investigated. The existence of a constant $\mu>0$ such that $\partial b\left[x_{1}, x_{2}, h\left(x_{1}, x_{2}\right)\right] / \partial u \leqq-\mu$ on the projection of $C$ in the $\left(x_{1}, x_{2}\right)$ plane, where $b\left[x_{1}, x_{2}, h\left(x_{1}, x_{2}\right)\right]=0$, is hypothesized. It is shown (under the preceding hypothesis and certain standard conditions) that there exists a nonvacuous closed subregion, $D$, of the $\left(x_{1}, x_{2}\right)$ plane and positive constants $\delta$ and $\epsilon_{0}$, all independent of $\epsilon$, such that if $C$ is within $\delta$ of the surface $u=h\left(x_{1}, x_{2}\right)$, then (1) has a unique solution $u=u\left(x_{1}, x_{2}, \epsilon\right)$ defined over $D$ for $0<\epsilon \leqq \epsilon_{0}$ which passes through $C$. Furthermore, $u\left(x_{1}, x_{2}, \epsilon\right) \rightarrow h\left(x_{1}, x_{2}\right)$ as $\epsilon \rightarrow 0+$ uniformly over every subset of $D$ which is a positive distance from the projection of $C$ in the $\left(x_{1}, x_{2}\right)$ plane. The analysis is similar to that employed in a recently announced result of Levinson and the author. (Received November 12, 1953.)

250t. E. R. Lorch: The projection of a linear functional on the manifold of measures.

Given an abstract set $\mathcal{E}$, let $\mathfrak{B}$ be a (real or complex) Banach space consisting of a set of bounded functions $f(x), x \in \mathcal{E}$, with norm $\|f\|=1$.u.b. $|f(x)| ; \mathscr{B}$ is also a lattice and hence contains $|f(x)|$ along with $f(x)$. By a positive measure is meant a bounded linear functional $F$ over $\mathfrak{B}$ with the properties: (i) $F f \geqq 0$ if $f(x) \geqq 0$ and (ii) if $f_{n}(x) \geqq 0$ and $f_{n}(x) \uparrow f(x)$, then $F f_{n} \rightarrow F f$. The theorem of Lebesgue and the work of Daniell are justification of this use of the term measure. An arbitrary real measure is the difference of positive measures; a complex measure is of the form $F_{1}+i F_{2}$ where $F_{1}, F_{2}$ are real. It is shown that there exists a projection $P$ on the space $\mathfrak{B}^{*}$ of linear functionals over $\mathfrak{B}$ such that the range of $P$ is the totality of measures. Thus each functional $F$ can be represented uniquely as the sum of a measure and a "nonmeasure." The existence of $P$ is established by transfinite arguments. Application is made to the case in which $\mathscr{B}$ is a commutative Banach algebra. If $F$ is the functional associated with a maximal ideal, either $P F=F$ or $P F=0$. The conditions determining the measure theoretic behavior of $F$ are topological in nature. (Received October 13, 1953.)

251. R. W. McKelvey: The solution of second order linear ordinary differential equations about a turning point of order two.

This paper is concerned with the behavior, for large values of a complex parameter $\lambda$, of the solutions of differential equations having the form $d u / d x-\left[p_{0}(x)+p_{1}(x) / \lambda\right.$ $\left.+p_{2}(x) / \lambda^{2}+\cdots\right] u=0$, where the $p_{i}(x)$ 's are suitably restricted bounded functions. $x$ is to vary over a real interval which contains a second order turning point of the equation-that is, a second order zero of $p_{0}(x)$. An algorithm is given, involving only the functions $p_{j}(x)$, which leads simply to the determination of expressions in negative powers of $\lambda$ which formally satisfy the differential equation. These formal solu- 
tions are then shown to represent true solutions in an asymptotic sense. The expressions seem to be of much simpler form than has been obtained previously. The algorithm resembles one employed by $\mathrm{R}$. E. Langer in his theory of the simple turning point, but with certain marked differences. The central role of the Bessel function $H_{1 / 3}$ in the first order theory is taken here by the Whittaker function $W_{k, 1 / 4}$, with index $k$ dependent upon $\lambda$. (Received December 29, 1953.)

\section{G. R. MacLane: Meromorphic functions with specified asymp- totic behavior.}

The following theorem is proved: Let $\left\{D_{n}\right\}$ be a finite or infinite sequence of domains in the z-plane such that: (1) the boundary of $D_{n}$ is a simple curve $\Gamma_{n}$ with both ends at $\infty$, (2) $\left(D_{m} \cup \Gamma_{m}\right) \cap\left(D_{n} \cup \Gamma_{n}\right)=0$ for $m \neq n$, and (3) $\left(D_{n} \cup \Gamma_{n}\right) \cap(|z| \leqq R)$ $=0$ for $n>n_{0}(R)$. Let $\left\{g_{n}(z)\right\}$ be any sequence of functions such that $g_{n}(z)$ is meromorphic in $D_{n} \bigcup_{\Gamma_{n}}$. Let $a$ and $p$ be any positive numbers. Then there exists a function $f(z)$, meromorphic in $|z|<\infty$, holomorphic in the complement of $\bigcup\left(D_{n} \cup_{\Gamma_{n}}\right)$, such that $f(z)-g_{n}(z)$ is holomorphic in $D_{n}$ and such that $\left|f(z)-g_{n}(z)\right|<a(1+|z|)^{-p}$ for $z \in D_{n}$. (Received November 14, 1953.)

\section{H. M. MacNeille: The approximation to surfaces by polyhe- drons. Preliminary report.}

Inscription as the basis for approximating surfaces by polyhedrons is well known not to provide a satisfactory approach to the problem of defining and determining the areas of surfaces. The present paper proposes an alternative approach to this problem suggested by an earlier paper of the author (Proc. Nat. Acad. Sci. U.S.A. vol. 27 (1941) pp. 71-76). (Received November 14, 1953.)

\section{Lawrence Markus: Quadratic ordinary differential equations} and their algebras.

The quadratic differential equations $\dot{x}^{i}=a_{j k}^{i} x^{i} x^{k}, i=1,2, \cdots, n\left(a_{j k}^{i}=a_{k j}^{i}\right.$ real or complex numbers), are determined up to affine equivalence by the tensor $a_{j k}^{i}$, which can also be realized as the multiplication table of a commutative linear algebra $u_{j} u_{k}=a_{j k}^{i} u_{i}$ for the basis $\left(u_{1}, \cdots, u_{n}\right)$. By obtaining all commutative algebras one determines all global quadratic differential systems first up to affine, then up to topological, equivalence. This program is carried out explicitly for low dimensions $n$. (Received November 12, 1953.)

\section{Joseph Milkman: Kolmogorov's axiom of continuity for rec-} tangular cylinder sets in Hilbert space.

An $n$-dimensional rectangle is the Cartesian product of $n$ sets of real numbers $A_{1}, A_{2}, \cdots, A_{n}$. Let $E_{n}$ denote an $n$-dimensional Euclidean space which is a subspace of the real Hilbert space $H$. The $n$-dimensional cylinder set $C(A)$ consists of all elements $x+h$ of $H$ such that $h \in A \subset E_{n} . C(R)$ is a Borel rectangular cylinder set if $R$ is an $n$-dimensional rectangle and a Borel set in $E_{n}$ for some $n$. Let $P(S)$ $=\int_{R} f_{1}\left(x_{1}\right) f_{2}\left(x_{2}\right) \cdots f_{n}\left(x_{n}\right) d x_{1} d x_{2} \cdots d x_{n}$ where $f_{i}\left(x_{i}\right)$ is a non-negative Lebesgue integrable function with the properties (1) $\int_{-\infty}^{\infty} f_{i}(t) d t=1$ and (2) There is an $m>0$ such that $|x|<m$ implies $\int_{-x}^{x} f_{i}(t) d t>x$. The following theorem is proved: If $S_{1}, S_{2}, \cdots$, $S_{n}, \cdots$ is a monotone decreasing sequence of Borel rectangular cylinder sets whose product is empty, then $\lim _{n \rightarrow \infty} P\left(S_{n}\right)=0$. (Received November 4,1953 .) 
256t. J. H. Monahan: Topological mappings by analytic quaternion functions.

The differential of a right analytic quaternion function $w$ in the sense of Fueter isgiven by $d w=R d \bar{x} i_{1}+S d \bar{x} i_{2}+T d \bar{x} i_{3}$. The functional determinant for such a function is then given by $\Delta=R(\bar{R} R \bar{R}+\bar{S} R \bar{S}+\bar{T} R \bar{T})+S(\vec{R} S \bar{R}+\bar{S} S \vec{S}+\bar{T} S \bar{T})+T(\vec{R} T \bar{R}+\overrightarrow{S T} \bar{S}+\bar{T} T \bar{T})$ and it may vanish in a regular point. Hence, unlike the case of the complex variable, the corresponding mapping is not always locally topological. If $R=0, w$ is a complexanalytic quaternion function $w=f_{1}\left(z_{1}, z_{2}\right)+i_{2} f_{2}\left(z_{1}, z_{2}\right)$ and the determinant reduces to $\Delta=(S S-T T)^{2}+(S T+T S)^{2}$ and can be zero only if $|S|=|T|$ and $S$ is perpendicular to $T$. In general $R=M+N$, where $M$ lies in the plane of $S$ and $T$ and $N$ is perpendicular to it. If $N=0, R=\beta S+\gamma T$ and $\Delta$ is always positive. If $M=0, \Delta=0$ when $N N$ $=(S \bar{S}+T \bar{T}) \pm\left(-(S \bar{T}-T \bar{S})^{2}\right)^{1 / 2}$. For $M, N \neq 0, \Delta=0$ when $4 S \bar{S} M \bar{M} \cos ^{2}(\phi-\alpha)$ $+4 T \bar{T} M \bar{M} \cos ^{2} \alpha \leqq-(S \bar{T}-T \bar{S})^{2}$ which is the equation of an ellipse. If $M \bar{M}$ is outside the ellipse, $\Delta$ is always positive; if $M \bar{M}$ is on the ellipse there is one value of $N \bar{N}$ for which $\Delta=0$ and if $M \bar{M}$ is inside the ellipse there are two values of $N \bar{N}$ for which $\Delta=0$. Over this ellipse we have a closed surface which bounds a three-dimensional region. Thus $\Delta$ is positive outside this surface, $\Delta=0$ on the surface, and $\Delta$ is negative inside the surface. (Received November 13,1953.)

257t. C. N. Moore: On relationships between Nörlund means for double series.

In this paper the theorems of Nörlund and Marcel Riesz concerning the consistency, inclusion, and equivalence of Nörlund means are generalized to double series. The results are analogous to those for single series, except that certain supplementary conditions enter into the discussion. (Received November 12,1953.)

258t. John Nash: $C^{1}$ isometric imbeddings of closed Riemannian nmanifolds in $E^{2 n}$.

The manifold should be differentiable and the components of the metric tensor should be continuous. First put a $C^{\infty}$ structure on the manifold and use Whitney's results to have a $C^{\infty}$ imbedding in $E^{2 n}$ which is shrunk proportionally until distances under the induced metric are everywhere less than under the intrinsic [given] metric. Now proceed to "stretch" the imbedding locally and directionally by adding [at each stage of this process] a spiral-like perturbation. The spiraling perturbation twists in the direction of the desired increase of the induced metric. By decreasing the amplitude but increasing the number of twists per unit distance we can indefinitely increase the accuracy and selectivity of the effect. So an infinite number of perturbations are added successively, the induced distances always being increased until the induced metric becomes the intrinsic metric in the limit. The process is such that first derivatives converge but not second derivatives, and its nature is such that it is easy to avoid introducing multiple points in the imbedding. To obtain imbeddings with more derivatives one expects to use a higher-dimensioned imbedding space. The author hopes to have results on the $C^{\infty}$ case before long. (Received December 21, 1953.)

\section{9t. A. B. Novikoff: On infinitely differentiable functions.}

A. Rosenthal (Proc. Amer. Math. Soc. vol. 4 (1953)) has given a simple construction for an infinitely differentiable function satisfying $f^{(n)}(0)=b_{n}, n=0,1,2, \cdots$, where the $b_{n}$ 's are an arbitrary prescribed sequence of real numbers. The author ex- 
hibits infinitely differentiable functions satisfying $f^{(n)}\left(a_{\nu}\right)=b_{\nu, n}, n=0,1,2, \ldots$; $\nu=1,2, \cdots, N$. The case of interpolating through a sequence $a_{\nu}, \nu=1,2,3, \cdots$, is solved if the $a_{v}$ 's have no finite limit point. This is the most that can be hoped for without restricting the prescribed values $b_{\nu, n}$. (Received October 19, 1953.)

260. L. E. Payne (p) and H. F. Weinberger: Upper and lower bounds for capacity and other functionals.

Let $D(u)$ be the Dirichlet integral of a harmonic function $u$ in an $N$-dimensional domain $D$ with boundary $B$. Let $n$ be the interior normal on $B$ and $r$ the distance from an arbitrary origin; let $u=O\left(r^{-1}\right)$ if $D$ extends to infinity. Upper and lower bounds for $D(u)$ are obtained by using the Green's identity $(N-2) D(u)=\int_{B}[2 r(\partial u / \partial r)(\partial u / \partial n)$ $\left.-r \partial r / \partial n \sum_{i=1}^{N}\left(\partial u / \partial x_{i}\right)^{2}\right] d \sigma$, already mentioned in another connection by Rellich (Math. Zeit. vol. 46 (1940) p. 635). If $u$ is the capacity function for $B$, the right-hand side reduces to $\int_{B} r(\partial r / \partial n)(\partial u / \partial n)^{2} d \sigma$ while the left-hand side with $N=3$ is $4 \pi$ times the capacity $C$. If $B$ is star-shaped so that $r \partial r / \partial n>0$ this permits one to obtain upper and lower bounds for $C$ by a method like that proposed by Wolf Gross (Rendiconti Lincei vol. 12 (1952) p. 496). Namely, if $\phi$ is any harmonic function of order $r^{-1}$ at $\infty$, then $\left(C-(1 / 4 \pi) \int(\partial \phi / \partial n) d \sigma\right)^{2} \leqq\left(1 / 16 \pi^{2}\right) \int_{B} r(\partial r / \partial n)(\partial u / \partial n)^{2} d \sigma \int_{B}\left((1-\phi)^{2} / r(\partial r / \partial n)\right) d \sigma$ $=(1 / 4 \pi) C \int_{B}\left((1-\phi)^{2} / r(\partial r / \partial n)\right) d \sigma$. Thus, if $\phi$ is chosen to make the integral on the right small, close bounds for $C$ are obtained. In particular, it is shown that Gross's estimate of $\int_{B}(\partial u / \partial n)^{2} d \sigma$ and hence his bounds for the capacity of the unit cube are incorrect. However, it is shown that his method gives the rigorous upper and lower bounds 0.668 and 0.627 . The former is an improvement of the best upper bound given in the recent book by G. Polya and G. Szegö. The method can be extended to general Dirichlet and Neumann problems in two or more dimensions. It has the computational advantage that even for exterior problems all calculations are to be performed on the boundary. (Received November 14, 1953.)

\section{1t. R. S. Phillips: The adjoint semi-group.}

Associated with each semi-group of linear bounded operators $T(s)$ defined on a Banach space $X$ to itself and possessing suitable continuity properties, there is an adjoint semi-group with like continuity properties defined on an "adjoint" Banach space $X^{+}$which is, in general, a proper subspace of the full adjoint space $X^{*}$. The choice of the subspace $X^{+}$is decisive; it is the largest domain over which the ordinary adjoint semi-group $T^{*}(s)$ has suitable continuity properties. Moreover, if $A$ denotes the infinitesimal generator of $T(s)$ and $A^{*}$ denotes the adjoint to $A$, then $X^{+}$can also be characterized as the closure of the domain of $A^{*}$. The infinitesimal generator $A^{+}$ of the adjoint semi-group defined on $X^{+}$turns out to be the maximal contraction of $A^{*}$ with domain and range in $X^{+}$. Both $A$ and $A^{+}$have the same spectral sets. As in the ordinary theory of adjoint spaces, it is possible to define an entire hierarchy of "adjoint" spaces corresponding to a given semi-group of operators. Likewise it can happen that the second "adjoint" space is equal to the original space (under the natural mapping). This situation occurs not only when $X$ is reflexive in the usual sense but, more generally, when the resolvent of $A$ is weakly compact. (Received October 7, 1953.)

262. M. H. Protter: Boundary value problems for equations of mixed type.

Uniqueness theorems are established for boundary value problems for an equation of the form $(*) K(z)\left(u_{x x}+u_{y y}\right)+u_{z z}=0$ where $K(z)$ is a monotone increasing func- 
tion of $z$ with $K(0)=0$. Let $S$ be a simple surface in the half-space $z>0$ with boundary curve $C$ on the plane $z=0$. Let $T$ be the set of points in the half-space $z<0$ with the property that the retrograde cone with vertex at a point of $T$ intersects the plane $z=0$ in a circle lying entirely in the interior of $C$. Consider a point $P(x, y, 0)$ interior to $C$. Construct the direct cone $K$ with vertex at $P$; denote its boundary by $L$. Designate by $\Gamma$ the intersection of $L$ with the boundary of $T$. Then the following problem is considered: prescribe boundary values on $S$ and the boundary of $T$ between $z=0$ and $\Gamma$. For this problem a uniqueness theorem is established for $(*)$. The condition on $K(z)$ is the same as that required in the corresponding two-dimensional case. Other problems in which the boundary of $T$ is not a characteristic surface can be treated by the same method. (Received November 6, 1953.)

\section{R. K. Ritt: Some remarks on the theory of summation.}

Banach, in his treatise, derived the Toeplitz theorem on the basis of the theory of linear functionals on a Banach space. In this paper, using methods due to Dieudonné and Schwartz, these results are extended. Consider the space of infinite sequences, $(S)$, and choose: (a) a locally convex topology for $(S)$; (b) a subspace $(E)$ of $(S)$ which includes the space of finite sequences, $\left(S_{f}\right)$. The linear functionals on $(E)$ which are continuous with respect to the topology chosen for $(S)$, and which, when restricted to $\left(S_{f}\right)$, coincide with usual summation are then studied. For different choices of (a) and (b), classes of linear functionals, which correspond, in special cases, to classical summation techniques are secured. (Received November 14,1953.)

264. L. B. Robinson: A functional equation with quasi analytical solution.

Let $\tilde{\omega}, \tilde{\omega}^{2}, \tilde{\omega}^{3}=1$. Write $u(\rho)=u_{0}+\lambda \int_{0}^{\rho}\left(1 /\left(1+\rho_{1}\right)^{2}\right) u\left(\rho_{1}^{2 p}\right) d \rho_{1}$. The solution is a meromorphic function whose poles are given by $\Delta_{1}(\lambda)=0$. Write $u(\tilde{\omega} \rho)=u_{0}$ $+\lambda \int_{0}^{\tilde{\omega} \rho}\left(1 /\left(1+\rho_{1}\right)^{2}\right) u\left(\rho_{1}^{2 p}\right) d \rho_{1}, u\left(\tilde{\omega}^{2} \rho\right)=u_{0}+\lambda \int_{0}^{\tilde{\omega}^{2} \rho}\left(1 /\left(1+\rho_{1}\right)^{2}\right) u\left(\rho_{1}^{2 \nu}\right) d \rho_{1}$. The solution is a meromorphic function whose poles are given by $\Delta_{2}(\lambda)=0$. Write the cyclic groups $\tilde{\omega}_{1}^{6 p}=1, \tilde{\omega}_{2}^{12 p}=1, \tilde{\omega}_{3}^{24 p}=1, \cdots u\left(\tilde{\omega}_{i} \rho\right)$ also has poles determined by $\Delta_{2}(\lambda)=0$. Now $\tilde{\omega}_{i} \rho \rightarrow \rho$ as $i$ grows greater. You would think that the determinant of $u\left(\tilde{\omega}_{i} \rho\right)$ would then $\rightarrow \Delta_{1}(\lambda)$ but it does not. It remains $\Delta_{2}(\lambda)$. This curious result is due to this fact: $u(x)$ admits the circumference of the unit circle as a singular line. And so the solution of our equation, continuous within the unit circle, ceases to be continuous outside. $u(x)$ may be called a quasi-analytic function. (Received November 10,1953.)

265. P. C. Rosenbloom: Approximation of eigenvalues for operators of the trace class.

The following lemma is proved: if $f$ is analytic and $|f| \leqq 1$ for $|z|<1,|f(0)|$ $=a>0,\left|f\left(z_{0}\right)\right|<\delta=\delta\left(a, r_{0}, \epsilon\right),\left|z_{0}\right|=r_{0}, \epsilon>0$, then $f$ has a zero in the circle $\left|z-z_{0}\right|<\epsilon$. The function $\delta\left(a, r_{0}, \epsilon\right)$ is estimated explicitly. We apply this to show that there is a function $\eta=\eta(M, r, \epsilon)$ such that if $K$ is an operator of the trace class on a Banach space $X$ into itself with trace norm $\leqq M,\|x\|=1,\left|\lambda_{0}\right| \leqq r$, and $\left\|x-\lambda_{0} K x\right\|<\eta$, then there is an eigenvalue of $K$ in the circle $\left|\lambda-\lambda_{0}\right|<\epsilon$. (Received November 14, 1953.)

\section{Hanan Rubin: The dock of finite extent.}

The scattering on a finite dock of a prescribed progressive wave in an ocean of infinite depth is considered. The required velocity potential $\Phi(\xi, \eta ; \tau)$ is to satisfy Laplace's equation $\Phi_{\xi \xi}+\Phi_{\eta \eta}=0$ for $\eta<0$ and the boundary conditions $\Phi_{\tau \tau}(\xi, 0 ; \tau)$ 
$+g \Phi_{\eta}(\xi, 0 ; \tau)=0$ for $|\xi|>\alpha$ and $\Phi_{\eta}(\xi, 0 ; \tau)=0$ for $|\xi|<\alpha$. Moreover, $\Phi(\xi, \eta ; \tau)$ is to have a logarithmic singularity of prescribed strength for $(\xi, \eta) \sim(\alpha, 0)$, and $\Phi(\xi, \eta ; \tau)$ $-A e^{\left(\sigma^{2} / \sigma\right) \eta} \cos \left(\left(\sigma^{2} / g\right) \xi+\sigma \tau-\gamma\right)$ is to satisfy a "radiation condition" for $\xi \sim \pm \infty$ with arbitrarily prescribed $A, \gamma$, and $\sigma$. Explicit formulas are given expressing $\Phi(\xi, \eta ; \tau)$ in terms of the solution to a minimum problem for the functional $F[w(x, y)]$ defined by $F[w]=\int_{y<0} \int\left(w_{x}^{2}+w_{y}^{2}\right) d x d y+\int_{-a}^{a}\left[w_{x}(x, 0)\right]^{2} d x$. The direct methods of the calculus of variations are employed to establish the existence and uniqueness of the solution to this minimum problem. The admissibility conditions require piecewise continuous $w_{x}(x, 0)$ for $|x|<a$. Consequently, after a uniformly convergent minimizing sequence of harmonic functions is obtained, estimates for the $x$-difference quotient near the boundary are derived from the "variational condition" in order to verify this admissibility condition for the limit function. The dependence of the reflection coefficient on the strength of the logarithmic singularity is noted. (Received November 12, 1953.)

\section{Walter Rudin: Natural boundaries of bounded analytic func-} tions.

Definitions: $B(D)$ is the set of all single-valued bounded analytic functions on $D$, a domain on the Riemann sphere. A point $x$ of $K$ (the complement of $D$ ) is removable if for every $f \in B(D)$ there exists a domain $D^{*}$ containing $x$ and $D$ and a function $f^{*} \in B\left(D^{*}\right)$ such that $f^{*}(z)=f(z)(z \in D)$. The other points of $K$ are essential. If all boundary points of $D$ are essential, $D$ is maximal. Results: (1) $x$ is removable if and only if there is some $r>0$ such that the intersection of $K$ with the closed disc of radius $r$ and center $x$ is a Painleve null-set (in the terminology of Ahlfors and Beurling, Acta Math. vol. 83 (1950) p. 107). (2) Every domain $D$ is contained in a smallest maximal domain $\Delta$; every $f \in B(D)$ can be extended to $\Delta$. (3) If $x$ is an essential boundary point of $D$, then there exists a function $f \in B(D)$ whose cluster set at $x$ consists of the entire unit disc, while $|f(z)|<1$ for all $z \in D$. (4) If $D$ is maximal (and only in this case), then some $f \in B(D)$ has the boundary of $D$ as its natural boundary. (Received November 12, 1953.)

\section{8t. Walter Rudin: Rings of bounded analytic functions.}

Let $D_{1}$ and $D_{2}$ be two maximal domains (see the preceding abstract for definitions) in the finite plane and suppose that there exist an isomorphic mapping $\psi$ of the ring $B\left(D_{1}\right)$ onto the ring $B\left(D_{2}\right)$. Then either $\psi(i)=i$ or $\psi(i)=-i$. In the former case it is shown that there exists a one-to-one conformal mapping $\phi$ of $D_{2}$ onto $D_{1}$ such that $f(\phi(w))=f^{*}(w)$ for $w \in D_{2}$, where $f^{*}=\psi(f)$ (if $\psi(i)=-i$, conjugates have to be introduced). It follows that $D_{1}$ and $D_{2}$ are conformally equivalent if and only if the rings $B\left(D_{1}\right)$ and $B\left(D_{2}\right)$ are algebraically isomorphic; this is a theorem of Kakutani and Chevalley whose proof is unpublished. The author's proof makes no use of the theory of normed rings: the identity function $I(z)=z$ is a member of the quotient field $F\left(D_{1}\right)$ of $B\left(D_{1}\right)$; extending $\psi$ to $F\left(D_{1}\right)$ and setting $\phi=\psi(I)$, it is shown directly that $\phi$ has the desired properties. (Received November 12, 1953.)

\section{I. J. Schoenberg: On multiply positive sequences and functions.}

Let $k$ be an integer $\geqq 2$ and let $P_{k}$ (the class of $k$-times positive functions) denote the class of functions $f(x),-\infty<x<\infty$, satisfying the following two conditions: 1. $f(x)$ is non-negative and $0<\int_{-\infty}^{\infty} f(x) d x<+\infty$. 2. If $x_{1}<x_{2}<\cdots<x_{n}, y_{1}<y_{2}<\cdots<y_{n}$, then det $\left\|f\left(x_{i}-y_{k}\right)\right\|_{1, n} \geqq 0$ and this should hold for every $n=1,2, \cdots, k$. Theorem 1 . If $f(x) \in P_{k}$ and $f(x)=0$ outside the interval $(-\pi / 2, \pi / 2)$, then its Laplace transform 
$F(s)=\int_{-\pi / 2}^{\pi / 2} \exp (-s x) f(x) d x$ has no zeros in the strip $|I s|<k$. Here $k$ is the best constant. Indeed the function $f_{k}(x)=(\cos x)^{k-2}$ for $-\pi / 2 \leqq x \leqq \pi / 2, f_{k}(x)=0$ outside this interval, is shown to be in $P_{k}$ while having a transform vanishing for $s= \pm k i$. This theorem is derived from an analogous theorem for zero-free sectors for polynomials whose coefficients form a $k$-times positive sequence in the sense of Fekete (Rendiconti di Palermo vol. 34 (1912) p. 3). The proof of this last theorem uses results on variation-diminishing transformations and Gaussian binomial coefficients. (Received November 14, 1953.)

\section{Jacob Schwartz: Essential spectra.}

The essential spectrum $\sigma_{e}(T)$ of an operator $T$ is the complement in its spectrum of the set of all points of finite multiplicity. The open complement of $\sigma_{e}(T)$ is the essential resolvent $\rho_{e}(T)$. Let $\rho_{\varepsilon}^{\prime}(T)$ be that component of $\rho_{e}(T)$ containing the point at infinity. Then, if $K$ is a compact (i.e., completely continuous) operator, $\rho_{e}^{\prime}(T+K)$ $=\rho_{0}^{\prime}(T)$. An example of Yood (Duke Math. J. vol. 18 (1951) p. 599) shows that $\rho_{e}(T+K) \neq \rho_{e}(T)$ is possible. As a special case it follows that $\rho_{e}(T+K)=\rho_{e}(T)$ if $T$ is a bounded or semibounded self-adjoint operator. The proof is based on the perturbation theory of spectra. (Received November 13,1953.)

\section{J. B. Serrin: On the Phragmén-Lindelöf principle for elliptic} partial differential equations.

Consider the equation $L(u)=a_{i j}(x) \partial^{2} u / \partial x_{i} \partial x_{j}+b_{i}(x) \partial u / \partial x_{i}=0$, where the coefficients are defined in a region $D$ in the half-space $x_{n} \geqq 0, x=\left(x_{1} \cdots x_{n}\right)$. Suppose that the boundary $\bar{D}$ of $D$ lies in a strip $0 \leqq x_{n} \leqq \sigma$, that $L$ is uniformly elliptic in $D$, and that there exists a decreasing function $p(r)$ such that $\left(\sum a_{i i}\right)^{-1}\left(\sum b_{i}^{2}\right)^{1 / 2} \leqq p(r)$, $r^{2}=\sum x_{i}^{2}$, and $\int_{0}^{\infty} p(r) d r<\infty$. Let $v$ be any function with the properties $(1) L(v) \leqq 0$, (2) $v \geqq 0$ on $D$, (3) $\lim _{x_{n} \rightarrow \infty} v / x_{n}=1$, uniformly in $x_{1}, \cdots, x_{n-1}$. (It is shown that functions $v$ always exist; in particular if all $b_{i}=0$ we may take $v=x_{n}$.) Theorem. Suppose $u=u(x)$ satisfies $L(u) \geqq 0$ in $D, u \leqq 0$ on $\bar{D}$. Then the limit $\alpha=\lim _{R \rightarrow \infty} M(R) / R$, where $M(R)=\max _{r=R} u(x)$, always exists (finite or infinite); moreover $\alpha$ is nonnegative and $(*) u<\alpha v$ throughout $D$. Equality cannot hold in $(*)$ unless $v=0$ on $\bar{D}, L(v)=0$, and in this case equality at any point in $D$ implies equality everywhere. This result is a strict analogue for elliptic equations of the classical PhragménLindelöf theorem for harmonic functions; the proof makes essential use of the work of Gilbarg and Hopf (Journal of Rational Mechanics and Analysis vol. 1, pp. 411-424). (Received November 13, 1953.)

\section{A. L. Shields: On the measure of a sum set.}

Let $G$ be a compact, connected, abelian, 2nd countable topological group (written additively) with Haar measure $m, m(G)=1$. Let $A, B \subset G$ be measurable and $m(A)$ $+m(B) \leqq 1$. Then, $m_{i}(A+B) \geqq m(A)+m(B)$, where $m_{i}$ is interior measure, and $A+B$ is the set of all $a+b, a \in A, b \in B$. The idea of the proof is to reduce the problem to a form of the $\alpha+\beta$ hypothesis of additive number theory. The following lemma is proven; let $G$ satisfy the hypotheses above and let $T$ be the transformation defined by $T(g)=g+g$. Let $A \subset G$ be measurable. Then $T^{-1}(A)$ is measurable, and $m\left(T^{-1} A\right)$ $=m(A)$. (Received November 9, 1953.)

273. George Springer ( $\mathrm{p}$ ) and Daniel Resch: On sums of schlicht functions. 
Let $\Sigma$ be the class of functions $f(z)=z+a_{1} / z+a_{2} / z^{2}+\cdots$ which are regular and schlicht in $|z|>1$ except for a simple pole at $z=\infty$. Let $h(z)=\alpha f(z)+\beta g(z)(f, g \in \Sigma$, $\alpha \geqq 0, \beta \geqq 0, \alpha+\beta=1)$ be schlicht in $|z|>R(h)$ but not in $|z|>r, r<R(h)$. Define $R=\max R(h)$ for all such $h$. Then it is proved that $1.381 \leqq R \leqq 2^{1 / 2}$. The mappings $f$ and $g$ for which $R(h)=R$ are mappings of $|z|>1$ onto slit regions of the $w$-plane. (Received November 18, 1953.)

\section{J. M. Stark: On value distribution of functions of two complex} variables.

The author considers analytic functions $f\left(z_{1}, z_{2}\right)$ of two complex variables in domains $M^{4}$ whose boundary $b^{3}$ consists of finitely many segments $j_{k}^{3}, k=1,2, \cdots, n$, of analytic hypersurfaces where $j_{k}^{3}=\sum_{\lambda} J_{k}^{2}(\lambda)$ are one-parameter families of segments $J_{k}^{2}(\lambda)$ of analytic surfaces. The sum of intersections $j_{m}^{3} \cap j_{n}^{3}, m \neq n$, forms the distinguished boundary surface $D^{2}$ of $M^{4}$. The author shows that certain information about the behavior of $f$ in $c^{3}=b^{3}-D^{2}$ leads to bounds for $\left|f\left(t_{1}, t_{2}\right)\right|$ where $\left(t_{1}, t_{2}\right)$ belongs to a subdomain of $M^{4}$. Using and generalizing results by Bergman (Mathematica vol. 14 (1938) p. 116 and Math. Zeit. vol. 39 (1934) p. 78), the author shows that if (I) in every boundary segment $j_{k}^{3}=\sum_{\lambda} J_{k}^{2}(\lambda)$ of $M^{4},|f| \leqq c_{k}(\lambda)$ and $\left|\partial f / \partial z_{\sigma}\right| \leqq A_{k}(\lambda), \sigma=1,2$, on a curve $z_{\nu}=Z_{\nu}(\lambda), \nu=1,2$, and (II) in every lamina $J_{h}^{2}(\lambda), f$ assumes no value more than once, then Koebe's theorem yields an upper bound for $\left|f\left(t_{1}, t_{2}\right)\right|$ for $\left(t_{1}, t_{2}\right)$ belonging to a certain subdomain of $M^{4}$. This bound is a function of $t_{1}, t_{2}, c_{k}(\lambda), A_{k}(\lambda)$, and some quantities which depend only upon $M^{4}$. (Received December 29,1953.)

275t. Gabor Szegö and Antoni Zygmund: On certain mean values of polynomials.

Let $f(z)$ be a polynomial of degree $n$ and let $\|f\|_{p}$ denote the magnitude of $f(z)$ in $L^{p},|z|=1$. Then $\|f\|_{q} /\|f\|_{p}<A n^{1 / p-1 / q}$ where $A$ depends on $p$ and $q, 0<p<q$. (In the case $p \geqq 1$ the inequality holds with an absolute constant $A$.) This inequality is discussed by two different methods and analogous inequalities are established for the case of an interval. For a wide class of curves the corresponding quotient as well as $\left\|f^{\prime}\right\|_{p} /\left\|_{f}\right\|_{p}$ are estimated. Finally A. Markov's theorem is proved for a square $S$ in the following sharper form: Let $|f| \leqq 1$ on $S$. Then $\left|f^{\prime}\right| \leqq O(1) \cdot \min \left\{n^{3 / 2}, n \delta^{-1 / 3}\right\}$ on $S$; here we assume that $S$ has the side 1 and $\delta$ is the distance of $z$ from the nearest vertex of $S$. (Received November 12, 1953.)

276t. C. T. Taam: An extension of Osgood's oscillation theorem for a nonlinear differential equation.

Consider $(\mathrm{A}):\left(m(t) x^{\prime}\right)^{\prime}+\sum_{i=1}^{k} f_{i}(t) x^{2 i-1}=0$, where $m^{-1}$ and $f_{i}$ are non-negative, Lebesgue-integrable in $[T, R]$ for every $R>T$. It is shown that on $T \leqq t<\infty$ if $m<m_{0}$, $f_{1}>f_{0}, m f_{i}>r$ for some $i, m_{0}, f_{0}$ and $r$ being positive constants, and if either all $m f_{i}$ are nondecreasing or all nonincreasing, then every solution of (A) is oscillatory and the amplitudes of the oscillations remain finite. If, furthermore, all $m f_{i}$ remain finite, the amplitudes of the oscillations have a positive lower bound. Moreover, the amplitudes vary monotonically, increasing when all $m f_{i}$ decreases monotonically, and decreasing in the opposite case. This generalizes an oscillation theorem of Osgood. Also given is a sufficient condition for the boundedness of the solutions of (A). Further generalizations are indicated. (Received November 12, 1953.)

277. C. T. Taam: Asymptotic relations between systems of differential equations. 
Consider (A): $y^{\prime}=A(x) y+f(x, y)$, where $A(x)=\left(a_{i j}(x)\right)$ is a $n \times n$ matrix and $y$ and $f(x, y)$ are vectors with components $y_{i}$ and $f_{i}$ respectively, $a_{i j}(x)$ and $f_{i}(x, y)$ (for each $y$ ) being complex-valued functions of the real variable $x$ and belonging to $L(0, R)$ for every $R>0$. Furthermore $f_{i}(x, y)$ is a continuous function of $y$ and $\|f(x, y)-f(x, Y)\| \leqq g(x)\|y-Y\|$ for all $y$ and $Y, g(x)$ being of $L(0, R)$ for every $R>0$. Let $Y$ be the solution of $Y^{\prime}=A(x) Y, Y(0)=I$. It is shown that if (a) $\|Y(x)\|=O(h(x))$, $h(x)$ being measurable on $0 \leqq x<\infty$, (b) $\|f(x, 0)\|(h(x))^{n-1} T(x)$ and $g(x)(h(x))^{n} T(x)$ belong to $L(0, \infty), T(x)=\exp \left[-\operatorname{Re} \int_{0}^{x} \operatorname{tr} A(t) d t\right]$, then, for any solution $y(x)$ of $(\mathrm{A})$, $y(x)=Y(x)(c+\epsilon(x))$ for some constant $c$ and $\epsilon(x) \rightarrow 0$ as $x \rightarrow \infty$. Moreover if (b) still holds after $n$ is replaced by $n+1$ and if $h(x)$ is nondecreasing, then $y(x)=Y(x) c$ $+\epsilon(x)$. By Phragmén-Lindelöf arguments, the results are extended to the complex domain. (Received November 12, 1953.)

278t. C. T. Taam: The boundedness of the solutions of an almost linear differential equation.

Let $1 / r(x), q(x)$, and $f(x, y)$ (for each fixed $y$ ) be real and of the class $L(0, R)$ for every $R>0$. For each $x \geqq 0$ let also $f(x, y)$ be a continuous function of $y$ satisfying the Lipschitz condition: $\left|f\left(x, y_{1}\right)-f\left(x, y_{2}\right)\right| \leqq g(x)\left|y_{1}-y_{2}\right|$, where $g(x)$ and $f(x, 0)$ belong to $L(0, \infty)$. It is shown that every solution of $\left(r y^{\prime}\right) y+q y=f$ is bounded if there exists a real-valued function $p=p(x)$ such that (1) $p$ belongs to $L(0, R)$ for every $R>0$; (2) $r p>0$ and absolutely continuous on $0 \leqq x<\infty$; (3) $p-q$ and $(r p)^{-1}(r p)^{\prime}-$ belong to $L(0, \infty),(r p)_{-}^{\prime}=\min \left((r p)^{\prime}, 0\right)$. A similar result holds when $r, q$, and $f$ assume complex values. (Received November 12, 1953.

\section{J. L. Ullman: On Faber expansions.}

Let $C$ be a simple analytic curve, $w=f(z)$ its normalized exterior mapping function, mapping $C$ onto $\Sigma_{\rho},|w|=\rho$, and let $C_{\lambda}$ be the image of $\Sigma_{\lambda},|w|=\lambda$. (a) If $F(z)$ is analytic inside and on $C$, then it has a unique expansion in the Faber polynomials $\left\{F_{n}(z)\right\}$ associated with $C$, say $\sum a_{n} F_{n}(z)$. This expansion converges inside $C_{\mu}$, the level curve first encountering a singularity of $F(z)$ as the index increases. (b) Besides proving (a), Faber (Math. Ann. vol. 57 (1903) pp. 389-408) correlated the analytic character of $\phi(t)=\sum a_{n} t^{n}$ with that of $F(z)$. In particular he showed that points on $\Sigma_{\mu}$ and $C_{\mu}$ which correspond under the mapping $w=f(z)$ are both regular or both singular. (c) In this direction, the author adds the theorem that if $\phi(t)$ is rational, then $F(z)$ is also. (d) As an application, consider a Faber expansion associated with $C=C_{\rho}$. If the series has coefficients assuming finitely many values, then $\phi(t)$ is either rational or has $|t|=1$ as a natural barrier, a result due to Szegö. Hence if $\rho<1$, by (b) and (c), $F(z)$ is rational or has $C$ as a barrier. (e) These remarks provide an essential simplification to L. Iliev, Series of Faber polynomials whose coefficients assume a finite number of values, Doklady Akad. Nauk SSSR vol. 40 (4) (1953) pp. 499-502. Furthermore, they apply with equal force to Faber expansions whose coefficients are integers. (Received November 14, 1953.)

\section{Daniel Waterman: Functions analytic in a half-plane.}

If $\Omega$ is a domain in the unit circle contained in the union of a Stolz angle $\eta$ and a circle $|z|<R<1$, and $\Omega_{\theta}$ is this region rotated about $z=0$ until 1 coincides with $e^{i \theta}$, Lusin defined the function $s(\theta)=\left\{\int_{\Omega} \int_{\theta}\left|\phi^{\prime}\right|{ }^{2} d x d y\right\}^{1 / 2}$; Zygmund and Marcinkiewicz showed that $A_{p, \eta}\|\phi\|_{p} \leqq\|s\|_{p} \leqq A_{p, \eta}\left\|_{\phi}\right\|_{p}\left(A_{\alpha, \beta} \ldots\right.$ denoting positive constants depending on the indicated parameters) provided that $\phi \in H_{p}$, for $p>0$. Littlewood and Paley defined $g^{*}(\theta)=\left\{(1 / \pi) \int_{0}^{\prime}(1-\rho) \partial \rho \int_{0}^{2 \pi}\left|\phi^{\prime}\left(\rho e^{i(\theta+t)}\right)\right|^{2} P(\rho, t)\right\}^{1 / 2}$, where $P(\rho, t)$ is 
the Poisson kernel. It has been shown (Zygmund, Littlewood, and Paley) that $A_{p}\left\|g^{*}\right\|_{p} \geqq\|\phi\|_{p}$ for $p>0$, and $A_{p}\|\phi\|_{p} \geqq\left\|_{g}\right\|_{p}$ for $p>1$. If one considers the Hille-Tamarkin class $\mathcal{H}_{p}$ in the half-plane $R(\sigma+i \tau)>0$ and defines $s(\tau)$ $=\left\{\int_{\Omega} \int_{\tau}\left|\phi^{\prime}(\sigma+i \beta)\right|{ }^{2} d \sigma d \beta\right\}^{1 / 2}$ where $\Omega_{\tau}$ is a region contained between $\sigma= \pm k \beta+\tau$, and $\left.g^{*}(\tau)=(1 / \pi) \int_{0}^{\infty} \sigma d \sigma \int_{-\infty}^{\infty}\left|\phi^{\prime}(\sigma+i \beta)\right|{ }^{2} P(\sigma, \tau-\beta) d \beta\right\}^{1 / 2}$ where $P(\sigma, \tau)=\sigma /\left(\sigma^{2}+\tau^{2}\right)$, then exact analogues of the cited results can be demonstrated. (Received November 13, 1953.)

\section{John Wermer: Maximal subalgebras.}

Let $B$ be a commutative Banach algebra, $A$ a closed subalgebra. Say that $A$ is maximal if there are no closed subalgebras of $B$ lying between $A$ and $B$. When $B$ is the algebra of all continuous functions on the circle $|\lambda|=1$, the author has shown that the closed subalgebra of $B$ generated by $\phi(\lambda)=\lambda$ is maximal. (On algebras of continuous functions, Proc. Amer. Math. Soc. vol. 4 (1953) pp. 866-869.) In this note a different method is used to show that the algebra of absolutely convergent Taylor series is a maximal subalgebra of the algebra of absolutely convergent Fourier series. The method used is applicable to a larger class of algebras. (Received November 14, 1953.)

282. F. M. Wright: Some results on the backward extension of $S$ sequences.

Using an $S$-fraction transformation theorem previously presented but as yet unpublished, the author first proves a result concerning the linear dependence of the $(k+1)$ terminating $S$-fractions involved in the first $k$ backward extensions of an $S$-sequence $\left\{\mu_{n}\right\}$ such that the $S$-fraction expansion of the formal power series $Q(w)$ $=\sum_{n=0}^{\infty} \mu_{n} w^{n+1}$ contains $2 k$ partial quotients. The transformation theorem mentioned above is then used to examine somewhat more closely than Wall (Trans. Amer. Math. Soc. vol. 31 (1929) pp. 91-116) has done the problem of extending backward an indeterminate Stieltjes moment sequence. Finally, this theorem is used to discuss the first backward extension of a Hausdorff moment sequence. (Received November 10, 1953.)

\section{Applied Mathematics}

\section{W. C. Carter: On Taylor-MacColl flow.}

The methods previously used to prove the existence of Taylor-MacColl flow may be improved by the introduction of more accurate approximations to the solution of the differential equations. These approximations allow the extension of the existence proof to include semi-cone angles ranging from 0 to 52.5 degrees and free stream Mach numbers from 1 to greater than 10. By the use of constants derived from Kopal's tables for supersonic conical flow these methods give new simple approximations in closed analytic form for supersonic right conical flow. In contrast to the usual linearized flow theory these approximations are more accurate for large cone angles and high free stream Mach numbers. (Received November 12,1953.)

284. R. C. DiPrima: A note on the asymptotic solutions of the equation of hydrodynamic stability.

The equation of hydrodynamic stability is $\phi^{i v}-2 \alpha^{2} \phi^{\prime \prime}+\alpha^{4} \phi+i \alpha R\left[(w-c)\left(\phi^{\prime \prime}-\alpha^{2} \phi\right)\right.$ $\left.-w^{\prime \prime} \phi\right]=0$, where the disturbance is given by the stream function $\Psi$ $=\phi(y) \exp [i \alpha(x-c t)]$. Tollmien (Z. Angew. Math. Mech. vols. 25 and 27 (1947) pp. 
33-50, 70-83), considering only real $y$ and real parameters $\alpha, R, c$, gave a system of asymptotic solutions of this equation. Recently, Wasow (Ann. of Math. vol. 58 (1953) pp. 222-253), considering general values of $y$ and the parameters, has given a complete theory of the manner in which the asymptotic solutions represent the fundamental solutions of the above equation. However, Wasow's error estimate for the derivative of the solution $v_{3}$ corresponding to the logarithmically singular solution $\phi_{2}$ of the reduced equation $(w-c)\left(\phi^{\prime \prime}-\alpha^{2} \phi\right)-w^{\prime \prime} \phi=0$ is not as good as that given by Tollmien for his solution $\phi_{2}^{*}$. By more detailed analysis of the work of each author it is shown that in the case of real parameters the derivative of the Wasow solution and the Tollmien solution agree to within the order of error given by Tollmien. (Received November 14, 1953.)

285. M. A. Hyman: Numerical determination of nonisentropic supersonic gas flows.

The case of stationary, inviscid flow past an axially-symmetric body is considered. A new "computation-by-characteristics" method is described, which seems advantageous for use with automatic computing machines. Certain new variables are introduced, in terms of which all required physical and engineering quantities can be simply expressed. The equations to be satisfied become simple algebraic relations between the differentials of the new variables. The absence of transcendental and other complicated operations makes "table look-ups" and special programming unnecessary. By a simple device the "three-characteristic" problem is reduced (with a gain in accuracy over standard "three-characteristic" methods) to a "two-characteristic" problem; as a result, programming time and computing time are saved, and space in the machine's memory is conserved. This method, developed in 1950-51, has been used in calculations undertaken since at the U. S. Naval Ordnance Laboratory, with good results. Though developed for axially-symmetric flow with shockwaves, the basic ideas of this new numerical method seem to carry over to many other "three-characteristic" problems. (Received November 3, 1953.)

\section{6t. Imanuel Marx: On the structure of recurrence relations.}

Let $y_{n-1}^{(1)}, y_{n}^{(1)}$ be the characteristic solutions of $d / d x[p(x) d y / d x]+[q(x)+\lambda] y=0$ corresponding to $\lambda_{n-1}, \lambda_{n}$, respectively, let $y_{n-1}^{(2)}, y_{n}^{(2)}$ be a pair linearly independent of the first, and let $z_{n-1}, z_{n}$ be an arbitrary pair of solutions. All recurrence relations between $z_{n-1}$ and $z_{n}$ are deducible from the pair $p A_{n}(x) d z_{n} / d x+B_{n}(x) z_{n}=z_{n-1}, p A_{n}(x) d z_{n-1} / d x$ $+C_{n}(x) z_{n-1}=s_{n} z_{n}, s_{n}$ a constant. The coefficients $A_{n}, B_{n}, C_{n}$ satisfy a system of firstorder nonlinear differential equations which combine to form a fourth-order linear equation for $A_{n}$. This is the same as the fourth-order equation satisfied by the product $z_{n} z_{n-1}$, so that $A_{n}=\sum_{j, k=1}^{2} a_{n}^{(i k)} y_{n}^{(i)} y_{n-1}^{(k)}$, and $B_{n} C_{n}$ are obtained from $A_{n}$ by quadratures while $s_{n}$ is easily computed. The constants $a_{n}^{(j k)}$ depend on the conditions that determine the characteristic values $\lambda_{n}$. A special case (Mathieu functions) was treated by E. T. Whittaker [J. London Math. Soc. (4) vol. 88 (1929)]. The factorization method of L. Infeld and E. T. Hull [Review of Modern Physics vol. 23] for recurrence relations corresponds to the simpler case $A_{n} \equiv$ constant, but in the differential equation $p(x) \equiv 1$ while $q(x)+\lambda$ is replaced by a more general function $r(x, \lambda)$. (Received November $12,1953$.

\section{7t. John Nash: Results on continuation and uniqueness of fluid flow.}

One can look at a gas in terms of the kinetic theory so that for each point and 
time there is a molecular velocity distribution. And a collision probability function enables the computation of the time derivative of the velocity distribution. One shows that initial conditions can be continued uniquely into the future so long as gross singularities of specified types do not occur. And one shows that the continuation from a $C^{k}$ initial pattern will remain $C^{k}$. The gross singularities are infinities and zeros of temperature and density. These results are relevant to the problem of turbulence which some have thought involved the nonexistence of higher derivatives of the flow quantities. It is hoped that an extension of these results to the continuum description [partial differential equations] of compressible viscous flow will be ready before long. (Received December 21, 1953.)

288t. R. H. Pennington: Surface instability in pulsating gas bubbles. I. Preliminary report.

The motion of an incompressible infinite fluid outside of a bubble of ideal gas is studied. The differential equations of motion and the correct boundary conditions are established for an ideal fluid, a viscous fluid, and for the case of surface tension. In the case of a nearly spherical bubble, a perturbation method is used which starts from the known radially symmetric solutions. The velocity field outside of the gas bubble can be obtained by linear superposition of velocity fields $\dot{r}_{0}(r, t)+\epsilon f(r, t) S_{n}(\theta, \phi)$, $\epsilon g(r, t) \csc ^{2} \theta\left(\partial S_{n}(\theta, \phi) / \partial \phi\right), \epsilon g(r, t)\left(\partial S_{n}(\theta, \phi) / \partial \theta\right)$, where the three functions determine the rate of change of the Eulerian polar coordinates, $r, \phi, \theta$, and where the $S_{n}$ are the $n$th order spherical harmonics. $\dot{r}_{0}(r, t)$ is a known radial velocity field of the unperturbed radially symmetric solution. $f(r, t)$ and $g(r, t)$ are connected by the relation $\partial\left(r^{2} f\right) / \partial r=n(n+1) r^{2} g$. The function $u(r, t)=(1 / n(n+1))\left\{\partial^{2}\left[r^{2} f(r, t)\right] / \partial r^{2}\right.$ $-f(r, t)\}$ is closely related to the vorticity of the motion and satisfies the differential equation $\left.u_{t}+\partial\left(\dot{r}_{0} u\right) / \partial r=(\mu / \rho)\left[u_{r r}-n(n+1) / r^{2}\right) u\right]$, where $\mu$ is a coefficient of viscosity and $\rho$ is the density of the fluid. (Received December 21, 1953.)

\section{9t. R. H. Pennington: Surface instability in pulsating gas bubbles.} II. Preliminary report.

The solutions in the problem of nearly spherical gas bubbles are developed into spherical harmonics and the time dependence of each harmonic component is studied. The differential equation for the amplitude is given. Both irrotational and rotational flows are admitted. If the surface of the bubble is described by the equation $r=R_{0}(t)+\epsilon A_{n}(t) S_{n}(\theta, \phi)$, then $A_{n}(t)$ is determined by the two differential equations $-\ddot{A}_{n} R_{0}-3 \dot{A}_{n} \dot{R}_{0}+(n-1) A_{n} \ddot{R}_{0}-(1 / \rho)(n-1)(n+1)(n+2) A_{n} T R_{0}^{-2}$ $+n(n+1) R_{0}^{n-1} \dot{R}_{0}\left[R_{0}^{3} \int_{R_{0}}^{\infty} \zeta^{n-3} u(\zeta, t) d \zeta-\int_{R_{0}}^{\infty} \zeta^{-n} u(\zeta, t) d \zeta\right]=(n+1)(\mu / \rho)\left[-n R_{0}^{-1} u\left(R_{0}, t\right)\right.$ $\left.+2(n+2) R_{0}^{-1} \dot{A}_{0}+4(n-1) A_{n} R_{0}^{-2} \dot{R}_{0}+2 n(n+1) R_{0}^{n-2} \int_{R_{0}}^{\infty} \zeta^{-n} u(\zeta, t) d \zeta\right]$ and $\mu\left[2(n+2) \dot{A}_{n}\right.$ $\left.-2(n-1) A_{n} R_{0}^{-1} \dot{R}_{0}+(n+1) u\left(R_{0}, t\right)+2(n+1) R_{0}^{n-1} \int_{R_{0}}^{\infty} \zeta^{-n} u(\zeta, t) d \zeta\right]=0$ where $T$ is the surface tension and $\mu, \rho$, and $u(r, t)$ are defined as in the preceding abstract, and $R_{0}(t)$ is known from the radially symmetric solution. The stability of the various modes is discussed and numerous special cases are treated. In the case of an irrotational nonviscous flow our differential equation for the $A_{n}(t)$ reduces to $\ddot{A}_{n} R_{0}+3 \dot{A}_{n} \dot{R}_{0}$ $-(n-1) A_{n} \ddot{R}_{0}+(1 / \rho)(n-1)(n+1)(n+2) R_{0}^{-2} A_{n} T=0$, an equation obtained recently by Plesset (On the stability of fluid flows with spherical symmetry, The Rand Corporation, Santa Monica, California). (Received December 21, 1953.)

290. S. I. Plotnick: Evaluation of constants in conformal representations. 
The Schwarz-Christoffel transformation, $d z=k \prod_{k=1}^{n}\left(\zeta-\xi_{k}\right)^{\alpha k / \pi-1} d \zeta=k f(\zeta) d \zeta$, maps the upper half $\zeta$-plane into a simple connected polygon. If $\xi_{k}$ in the $\zeta$-plane corresponds to two points $P_{k}, Q_{k}$ in the z-plane and $\zeta=\xi_{k}$ is a simple pole of $f(\zeta)$ (z-plane contains a strip), then it is proved that $k=\operatorname{dist}\left(P_{k}, Q_{k}\right) / \pi_{i} R\left(\zeta=\xi_{k}\right), R$ denoting residue and dist $\left(P_{k}, Q_{k}\right)$ denoting the vector distance between the two points $P_{k}$ and $Q_{k}$. (Received November 2,1953.)

\section{Domina E. Spencer: Maxwell's equations and the new electro-} dynamics.

A previous paper (D. E. Spencer, Field theory and the new electrodynamics, Bull. Amer. Soc. Abstract 59-6-672) has presented a single equation for the force between moving charges. The purpose of the present paper is to clarify the relationship between the Maxwell equations and the new electrodynamics. It is shown that the Maxwell equations can be obtained as a special case if two assumptions are made: I. The Ampere equation for the force between current elements is replaced by the Grassmann equation. II. A moving charge is interchangeable with a current element if both represent the same rate of transport of charge. The first assumption is rigorously correct only for closed circuits. The second assumption cannot be made if the theory is to be expressed in terms of relative velocities. Once this assumption is introduced, invariance under Galilean transformations is lost. For most engineering problems Assumptions I and II are satisfactory approximations. From the point of view of the new electrodynamics, the Maxwell equations are a useful approximation, but they constitute a shaky foundation on which to base all of electricity and magnetism. (Received November 14, 1953.)

292. A. H. Van Tuyl: Some exact solutions in electrostatics and hydrodynamics.

The following result of Ernst Neumann (J. Reine Angew. Math. vol. 120 (1899) pp. 277-304) is studied in special cases and extended: Given a sphere $S$ of radius $R$, and a system of conductors $B$ which is transformed into itself by inversion with respect to $S$, let $U(P)$ be the potential function of $B$ at a point $P$ (equal to unity on $B$ and regular outside), let $C$ be the capacity of $B$ with respect to the infinite sphere, and let $r$ be measured from the center of $S$; then the potential function of the composite conductor $B+S$ is $U(P)+R / r-(R / r) U\left(P^{\prime}\right)$, where $P^{\prime} \equiv(R / r)^{2} P$, and its capacity is $C+R-R U(0)$. This result gives the potential function of certain conductors which do not seem to have been considered explicitly in the past. They include: (1) a torus and a sphere intersecting orthogonally; (2) a torus and two spheres, all mutually orthogonal; (3) special configurations of four and five spheres. Using the generalization of Neumann's result to $n$-dimensional Euclidean space, and results of Payne and Weinstein (L. E. Payne, Quarterly of Applied Mathematics vol. 10 (1952) pp. 197-204), solutions in hydrodynamics and elasticity are obtained in the axially symmetric cases. (Received November 18, 1953.)

\section{Geometry}

293t. Eugenio Calabi: The variation of Kähler metrics. I. The structure of the space.

Let $M^{n}$ be a compact complex manifold admitting at least one Kähler metric $g_{\alpha \beta^{*}}$; one considers the closed, exterior form $\omega_{0}=(-1)^{1 / 2} g_{\alpha \beta^{*}} d z^{\alpha} \wedge d z^{\beta^{*}}$ and its (the principal) cohomology class $(\omega)$. If $R_{\alpha \beta^{*}}$ is the Ricci tensor, then $\Sigma_{0}=(-1)^{1 / 2} R_{\alpha \beta^{*}} d z^{\alpha}$ 
$\wedge d z^{\beta^{*}}$, the Ricci form, is closed and cohomologous to $2 \pi$ times the first Chern class. Theorem: Given any closed exterior form $\Sigma$ of type $(1,1)$, of class $C^{\infty}$, cohomologous to $2 \pi$ times the first Chern class, there exists a unique Kähler metric of class $C^{\infty}$, whose principal cohomology class is $(\omega)$, and whose Ricci form equals $\Sigma$. With the aid of this theorem one considers the set of all Kähler metrics of class $\bigodot^{\infty}$, whose principal class is $(\omega)$. It is an infinite-dimensional "open manifold," in which there is a natural Riemannian metric $d S^{2}=(\eta, \eta)$ where $\eta$ is the first variation of any given $\omega$ and $(\phi, \psi)$ is the integrated inner product of two forms $\phi, \psi$. This manifold has constant, positive curvature, unique geodesics of bounded length between any pair of points, and its completion consists of the positive semidefinite Kähler metrics defining the same principal class. (Received October 28, 1953.)

294. Eugenio Calabi: The variation of Kähler metrics. II. A minimum problem.

Let $M^{n}$ be a compact, $n$-dimensional Kähler manifold, and $(\omega)$ its principal cohomology class (see previous abstract). For each principal Kähler form $\omega \in(\omega)$ let $\Sigma$ be its Ricci form, $(\Sigma, \Sigma)$ the norm of that form, $R=-2 \Lambda \Sigma$ the scalar curvature, and $H$ the operator projecting forms into their harmonic part. The following constants depend only on $(\omega)$, not on $\omega: \int_{M^{n} \omega^{n}}=V, H R=2 n V^{-1} \int_{M^{n} \omega^{n-1}} \wedge \Sigma,(H \Sigma, H \Sigma)=V(H R)^{2}$ $-n(n-1) \int_{M^{n} \omega^{n-2}} \wedge \Sigma^{2}$. Theorem $1: \Sigma$ is harmonic with respect to $\omega$ if and only if $R$ is a constant; if this is the case, and for fixed $(\omega)$, the metric is determined within any transformation due to the complex Lie group of analytic deformation of $M^{n}$, and $(\boldsymbol{\Sigma}, \boldsymbol{\Sigma})$ is minimized. The converse is implied for most complex manifolds by Theorem 2: If, for a certain $\omega \in(\omega),(\Sigma, \Sigma)$ is minimized, then the contravariant vector $g^{\alpha \beta^{*}} R_{\alpha \beta^{*}}$ is holomorphic; this implies that $\Sigma$ is harmonic if $M^{n}$ has no analytic deformations. The existence of an $\omega \in(\omega)$ minimizing $(\Sigma, \Sigma)$ is, except in special cases, still unproved. (Received October 28, 1953.)

\section{5t. John DeCicco: Some theorems in the geometry of the Kasner turbine group in the complex domain.}

Under a representation $R$, every point of a complex projective three-dimensional space $S_{3}$ is represented as a lineal element of the projective extension of a complex Euclidean plane. By $R$, the straight lines and planes of $S_{3}$ correspond to turbines and flat fields in the opulence of lineal elements. The Kasner turbine group $G_{15}$ is isomorphic to the complete projective group in $S_{3}$. The conjugate operation $N$ in the opulence is represented as a special correlation in $S_{3}$. To every transformation $\Sigma$ of $G_{15}$, there corresponds a single conjugate transformation $\Sigma=N \Sigma N$ of $G_{15}$, such that $\boldsymbol{\Sigma}$ and $\bar{\Sigma}$ carry conjugate turbines into conjugate turbines. The self-conjugate transformations $\Sigma=\bar{\Sigma}$ of $G_{15}$ form the Lie circular group $L_{10}$. If $\Sigma \neq \bar{\Sigma}$, then $\Sigma \bar{\Sigma}^{-1}$ leaves invariant the lineal elements of two conjugate turbines $T_{0}$ and $\bar{T}_{0}$. If $\Sigma \neq \bar{\Sigma}$, all the circles which pass through the lineal elements of $T_{0}$ and $\bar{T}_{0}$ are converted into circles by $\Sigma$. Certain subgroups of $G_{15}$, which include the Moebius and Laguerre inversive groups, are also studied. The representation $R$ leads to the concept of conjugate differential equations and also to a new theory of algebraic differential equations invariant under the Lie group $L_{10}$. (Received November 16, 1953.)

296. E. F. Gillette: $A$ generalization of the Four Vertex Theorem for ovals on a surface. I.

Given in the plane a closed convex curve without double points along which the 
curvature is a continuous function of the arc length. Then the classical Four Vertex Theorem states that the curvature assumes at least four extrema. Replacing the concept of curvature by that of geodesic curvature and giving a suitable definition of convexity, the Four Vertex Theorem can be generalized for ovals on surfaces of nonpositive Gaussian curvature. The proof is based on an extension of the following lemma of E. Schmidt: Given in the plane an arc $C$ with continuous curvature and of length $L$ which together with the chord joining its end points bounds a convex domain. Let $\bar{C}$ be a second arc (not necessarily plane) of the same length $L$. If for every value of the arc length $s$ as parameter the curvature $k(s)$ of $C$ is greater than or equal to the curvature $\bar{k}(s)$ of $\bar{C}$, then the length $d$ of the chord of $C$ is smaller than or equal to the length $\bar{d}$ of the chord joining the end points of $\bar{C} .(\bar{d}=d$ only if $\bar{C}$ is congruent to $C$.) The Four Vertex Theorem for ovals on surfaces of positive Gaussian curvature will be treated in a subsequent paper. (Received November 14, 1953.)

\section{L. W. Green: Geodesic instability.}

Let $M$ be a complete, simply-connected, $n$-dimensional Riemannian manifold of class $C^{r}(r \geqq 4)$ with the property that no geodesic of $M$ contains two mutually conjugate points. Letting $K(\beta, P)$ denote the curvature at $P$ of the geodesic plane element tangent to the bivector $\beta$, assume further that $K(\beta, P) \geqq-A^{2}$ for some constant $A$ and all $P, \beta$ in $M$. The principal result is: any two geodesic rays of $M$ with the same initial point must diverge (i.e., the distance from a point on one to the other geodesic approaches infinity with its distance from the point of intersection). This is an extension of the author's previous result for surfaces and has several new consequences. In particular, if $R$ is a Riemannian $n$-torus such that no geodesic contains a point focal to a transverse geodesic hyperplane, then $R$ is locally Euclidean. It is also proved that the geodesic flow is topologically transitive on a wide class of $n$-dimensional manifolds (not necessarily compact) whose universal covering manifolds satisfy the hypotheses on $M$. (Received November $10,1953$. )

\section{A. J. Hoffman (p), Morris Newman, E. G. Straus, and Olga} Taussky: The number of absolute points of a correlation.

Let $\rho$ be a correlation of a finite projective plane $\pi$ with $n+1$ points on a line. Let the collineation $\rho^{2}$, regarded as a permutation of the points of $\pi$, be expressible as a product of disjunct cycles of length $d_{1}, d_{2}, \cdots, d_{r}$. The principal results are: (1) bounds on the number of absolute points of $\rho$ in terms of arithmetic properties of $n$ and $d_{1}, d_{2}, \cdots, d_{r}$; (2) relations between the number of absolute points of $\rho$ and the number of absolute points of various odd powers of $\rho$. These results, obtained from a study of irreducible factors of the characteristic polynomial of a suitably chosen incidence matrix for $\pi$, improve a portion of the work of R. Baer and R. W. Ball concerned with the same problems. The present method applies also to correlations of symmetric group divisible designs, a class of combinatorial configurations including the finite projective planes. (Received November 14, 1953.)

\section{LOGIC AND FOUNDATIONS}

299. M. D. Davis: Pseudocomplements of recursively enumerable sets. Preliminary report.

Let $\{n\}=[x|(E y) T(n, x, y)|$ where $T$ is Kleene's enumeration predicate, so that 
$S$ is recursively enumerable if and only if there exists $n_{0}$ such that $S=\left\{n_{0}\right\}$. Recursive functions $\vee, \wedge$ may be defined such that $\{m \bigvee n\}=\{m\} \cup\{n\} ;\{m \wedge n\}=\{m\}$ $\cap\{n\}$. Let $\mathcal{L}$ be an $\omega$-consistent logic adequate for recursive arithmetic. Then, as is well known, $\{n\}=[x \mid \vdash \mathcal{L} x \in\{n\}$. $]$ However, if $\{n\}$ is not recursive, $\{\vec{n}\} \neq[x \mid \vdash \mathcal{L} x$ $\notin\{n\}$ ], this being the substance of Gödel's theorem. Let a recursive function $N(n)$ be determined such that $\{N(n)\}=[x \mid \vdash \mathcal{L} \in \notin\{n\}] .\{N(n)\}$ is called a pseudocomplement of $\{n\}$. Then: (1) $\{n \wedge N(n)\}=\varnothing(\varnothing$, the empty set). (2) $\{N(m \vee n)\}$ $=\{N(m) \wedge N(n)\}$. (3) $\{N(m \wedge n)\} \supset\{N(m) \bigvee N(n)\} . \quad$ (4) $\quad\{N(N(n))\}=\varnothing$. (5) $\{m \wedge N(m \wedge n)\} \subset\{N(n)\}$. (6) If $R$ is recursive, there exists $n$ such that $\{n\}=R$, $\{N(n)\}=\widehat{R}$. A consequence of (3), (4), and (5) is that $\{N(n)\} \subset\{N(n \wedge N(n))\}$ $\subset\{\bar{n}\}$, so that $\varnothing$ has arbitrarily small pseudocomplements. Also, it follows from (1), (2), and (4) that $\{N(n \bigvee N(n))\}=\varnothing$, i.e. the direct attempt to employ $N$ itself to explore the "undecidable" region fails. (Received November 13,1953.)

300t. W. J. Feeney: Certain unsolvable problems in the theory of cancellation semi-groups.

The nonexistence of algorithms for determining whether $S$ is trivial, isomorphic to $S^{\prime}$, abelian, finite, a group, imbeddable in a group, or has a solvable word problem, where $S$ and $S^{\prime}$ are arbitrary finitely presented cancellation semi-groups, follows from a theorem concerning invariant (preserved under isomorphism) properties of cancellation semi-groups. Finitely presented cancellation semi-groups in which the cancellation law is superfluous will be called special. Theorem: Let $\mathrm{P}$ be an invariant property of cancellation semi-groups such that (1) there exists a special cancellation semi-group $S_{0}$ which cannot be imbedded in any cancellation semi-group having the property $\mathrm{P}$, and (2) there exists a special cancellation semi-group which has the property $\mathbf{P}$. Then there is no algorithm for deciding in a finite number of steps whether an arbitrary finitely presented cancellation semi-group possesses the property $\mathrm{P}$. In proof, let $S_{1}$ be Turing's special cancellation semi-group with unsolvable word problem. For each two words $G, H$ of $S_{1}$ form $S_{G, H}$ by adjoining to the free product of $S_{0}, S_{1}$, and $S_{2}$ generators $a_{x}, b_{x}, c_{x}, d_{x}$ and relations (1) $x a_{x} b_{x} a_{x} G c_{x} d_{x} c_{x}$ $=b_{x} a_{x} b_{x} H d_{x} c_{x} d_{x}$, (2) $b_{x} a_{x} b_{x} G d_{x} c_{x} d_{x}=a_{x} b_{x} a_{x} H c_{x} d_{x} c_{x}$, (3) $a_{x} a_{x} b_{x}^{2} a_{x} G c_{x} d_{x}^{2} c_{x}=b_{x} a_{x}^{2} b_{x} H d_{x} c_{x}^{2} d_{x}$, (4) $b_{x} a_{x}^{2} b_{x} G d_{x} c_{x}^{2} d_{x}=a_{x} b_{x}^{2} a_{x} H c_{x} d_{x}^{2} c_{x}$, (5) $b_{x} b_{x} a_{x}^{3} b_{x} G d_{x} c_{x}^{3} d_{x}=a_{x} b_{x}^{3} a_{x} H c_{x} d_{x}^{3} c_{x}$, (6) $a_{x} b_{x}^{3} a_{x} G c_{x} d_{x}^{3} c_{x}$ $=b_{x} a_{x} b_{x}^{3} H d_{x} c_{x}^{3} d_{x}$, (7) $a_{x} b_{x}^{4} a_{x} G c_{x} d_{x}^{4} c_{x} c_{x}=b_{x} a_{x}^{4} b_{x} H d_{x} c_{x}^{4} d_{x}$, (8) $b_{x} a_{x}^{4} b_{x} G d_{x} c_{x}^{4} d_{x}=a_{x} b_{x}^{4} a_{x} H c_{x} d_{x}^{4} c_{x}$, (9) $b_{x} a_{x}^{\mathbf{s}} b_{x} G d_{x} c_{x}^{\mathbf{b}} d_{x} d_{x}=a_{x} b_{x}^{\mathbf{b}} a_{x} H c_{x} d_{x}^{\mathbf{b}} c_{x}$, (10) $a_{x} b_{x}^{\mathbf{b}} a_{x} G c_{x} d_{x}^{\mathbf{s}} c_{x}=b_{x} a_{x}^{\mathbf{b}} b_{x} H d_{x} c_{x}^{\mathbf{s}} d_{x}$, for each generator $x$ of $S_{0}$ and $S_{1}$. It can then be shown that $S_{G, H}$ possesses the property $\mathrm{P}$ if and only if $G=H$ in $S_{1}$. (Received January 7,1954 .)

\section{1t. Hao Wang: Undecidable sentences generated by semantic para- doxes.}

If the notion of truth or the relation of designation of a system is definable in the system itself, we get some semantic paradoxes in the system. There are systems $S$ which satisfy the following conditions: (1) the notions of truth and designation of current number theory $Z$ can be defined in $S$; (2) $S$ is omega-consistent; (3) $S$ contains $Z$ as a part. For example, the von Neumann-Bernays set theory is demonstrably one such system if we delete the axiom of infinity. These systems $S$ have models in $Z$ (or more accurately in omega-consistent extensions of $Z$ ). In particular, the definitions of truth and designation of $Z$ in $S$ also have models in $Z$. Using these facts, the author gets undecidable sentences in $S$ which correspond to the paradoxes of Richard and Epimenides. (Received October 16, 1953.) 


\section{Statistics and Probability}

\section{K. L. Chung: On the Robbins-Monro procedure.}

Asymptotic properties are established for the Robbins-Monro procedure (Ann. Math. Statist. vol. 22 (1951) pp. 400-407) of stochastically solving the equation $M(x)=\alpha$. Two disjoint cases are treated in detail. The first may be called the "bounded" case, in which the assumptions are similar to the second case of Robbins and Monro. The second may be called the "quasi-linear" case which restricts $M(x)$ to lie between two straight lines with finite and nonzero slopes but postulates only the boundedness of the moments of $Y(x)-M(x)$ (see loc. cit. for notations). In both cases it is shown how to choose the sequence $\left\{a_{n}\right\}$ in order to establish the correct order of magnitude of the moments of $x_{n}-\theta$. Asymptotic normality of $a_{n}^{-1 / 2}\left(x_{n}-\theta\right)$ is proved in both cases under a further assumption. The case of a linear $M(x)$ is discussed to point up other possibilities. The statistical significance of our results is sketched. (Received September 17, 1953.)

303t. K. L. Chung and Paul Erdös: Probability limit theorems assuming only the first moment. II.

This is a sequel to Probability limit theorems assuming only the first moment. I, Memoirs of the American Mathematical Society, no. 6. The non-lattice case is taken up here. In general additional device is needed since no superfluous assumptions are made; in particular, there is no discrimination against a singular distribution. (Received, November $9,1953$.

\section{Topology}

\section{4t. J. D. Baum: A symbolic transformation group.}

A symbolic transformation group, an extension of the Morse minimal set [M. Morse and G. A. Hedlund, Symbolic dynamics, Amer. J. Math. vol. 60 (1938) pp. 815864 ], is defined over the phase group of lattice points of the plane. It is shown that this transformation group is a minimal set, the orbit closure of a nonperiodic, almost periodic, point, which decomposes into a set of pairwise separated orbits and a set which is the union of pairs of points asymptotic relative to a half-space of the phase group. The asymptotic relations among eight selected points are completely investigated. (Received October 29, 1953.)

\section{5t. J. D. Baum: Asymptoticity in topological dynamics.}

If $(X, T)$ is a transformation group [definitions as in Gottschalk and Hedlund, The dynamics of transformation groups, Trans. Amer. Math. Soc. vol. 65 (1949) pp. 348-359], $X$ a uniform space, and $P$ a replete semigroup in $T, P \neq T$, then $x, y \in X$, $x \neq y$, are said to be $P$-asymptotic if for each index $\gamma$ of $X$ there exists $q \in P$ such that $x q p \in y q p \gamma$ for all $p \in P$. If $A$ is closed invariant in $X, x \in A, x$ is said to be $P$-asymptotic to $A$ if for each neighborhood $U$ of $A$ there exists $q \in P$ such that $x q P \subset U$. These definitions free the notion of asymptoticity from restriction to dynamical systems and iterated homeomorphisms. The $P$-limit set of a point $x \in X, P_{x}$, is a generalization of $\alpha$ - and $\omega$-limit sets by the above authors and is used extensively to prove the following (where $T$ is separable generative): (1) $P_{x}$ is the smallest set to which $x$ can be $P$-asymptotic, (2) $x$ is $P$-asymptotic to $P_{x}$ if and only if $x \notin P_{x},(3)$ if $T=I^{n}, I$ the integers, and $X$ is compact metric and dense-in-itself, then there exist $P$-asymptotic points, where $P$ is a half space of $T$. Two points are totally asymptotic provided they 
are $P$-asymptotic for every replete semigroup $P$ in $T, P \neq T ; x$ and $y, x \neq y$, are said to be compactly asymptotic if for each index $\gamma$ of $X$ there exists $K$ compact in $T$, such that $t \notin K$ implies $x t \in y t \gamma$. It is shown that (1) if $T$ is separable generative then total asymptoticity implies compact asymptoticity and (2) if $T$ is abelian compact asymptoticity implies total asymptoticity. (Received October 29, 1953.)

\section{R. H. Bing: Decompositions of Euclidean spaces.}

Suppose $G$ is a monotone upper semicontinuous decomposition of $S^{2}$ such that $G$ with respect to its elements as points is topologically equivalent to $S^{2}$. It is shown that there is a deformation $f(x, t) \quad\left(x \in S^{2}, 0 \leqq t \leqq 1\right)$ of $S^{2}$ onto itself shrinking elements of $G$ onto distinct points such that for $0<a<1, f(x, t)(0 \leqq t \leqq a)$ is an isotopy. This theorem does not generalize to $S^{3}$ because there is a monotone upper semicontinuous decomposition of $S^{3}$ onto itself such that two elements of the decomposition are linking circles. In fact if $W$ is a finite collection of mutually exclusive compact continua in $E^{3}$ such that no one of them separates $E^{3}$, there is a monotone upper semicontinuous decomposition of $E^{3}$ onto itself that has the continua of $W$ as elements. Suppose $G^{\prime}$ is a monotone upper semicontinuous decomposition of a solid cube $C$ such that each point on the interior of $C$ is an element of $G^{\prime}$ and the other elements of $G^{\prime}$ constitute in the decomposition space a set topologically equivalent to $S^{2}$ (or a dendron). Then it is shown that the decomposition space $G^{\prime}$ is topologically equivalent to $C$ (or $S^{3}$ ). (Received November 14, 1953.)

\section{Raoul Bott: On manifolds all of whose geodesics are closed.}

The following propositions are proved about simply-connected manifolds $M$ with the above property (satisfying also certain regularity conditions concerning the way in which the geodesics are closed). I. The integral cohomology ring of $M$ is isomorphic to a truncated polynomial ring (i.e., apart from the identity, $H(M)$ is generated by a single class $\chi(M)$ subject to only one relation which is of the type $\chi^{n}(M)=0, n>1$ ). II. If $\sigma(M)$ is the associated space of closed geodesics on $M$ (obtained by fibering the tangent bundle of $M$ by the closed geodesics) the Poincare series of the circular connectivities of $M$ (see Marston Morse, Calculus of variations in the large, Amer. Math. Soc. Colloquium Publications, vol. 18), given by $P(M ; z)=P\{\sigma(M) ; z\} z^{\mathrm{s}}\left(1-z^{2 t}\right)^{-1}$ where $P\{\sigma(M) ; z\}$ is the mod 2 Poincare polynomial of $\sigma(M) ; 1+s=\operatorname{dim} \chi(M)$; and $t+1=\operatorname{dim}(M)$. This result interprets and generalizes formulae obtained by Morse for the circular connectivities of the $n$-sphere. (Received November 14,1953.)

\section{J. W. Brace: The closure of weakly compact transformations.}

The set of transformations (weakly compact transformations) taking the bounded sets of a linear topological space $E$ into the weakly compact sets of a linear, locally convex, topological space $F$ is closed in the topology of uniform convergence on the bounded sets of $E$, if $F$ is complete in the weak topology, the original topology, or any topology coarser than the original topology and finer than the weak topology. In considering a net of weakly compact transformations converging in the topology of uniform convergence on the bounded sets of $E$, and a fixed bounded set in $E$, it is observed that the relatively weakly compact images of the bounded set form what can be called a "Cauchy net of sets" in the original topology on $F$. When $F$ has the desired completeness property the "Cauchy net of sets" converges in the weak topology to a relatively weakly compact set. This limit set is the image of the fixed bounded set under the limit transformation; thus establishing the above theorem. (Received November 10, 1953.) 


\section{C. E. Burgess: Some theorems on n-homogeneous continua.}

A point set $M$ is said to be $n$-homogeneous if for any $n$ points $x_{1}, x_{2}, \cdots, x_{n}$ of $M$ and any $n$ points $y_{1}, y_{2}, \cdots, y_{n}$ of $M$ there is homeomorphism of $M$ onto itself that carries $x_{1}+x_{2}+\cdots+x_{n}$ into $y_{1}+y_{2}+\cdots+y_{n}$. A point set $M$ is said to be nearly $n$-homogeneous if for any $n$ points $x_{1}, x_{2}, \cdots, x_{n}$ of $M$ and any $n$ open subsets $D_{1}, D_{2}, \cdots, D_{n}$ of $M$ there exist (1) $n$ points $y_{1}, y_{2}, \cdots, y_{n}$ such that $y_{i} \subset D_{i}$ $(1 \leqq i \leqq n)$ and (2) a homeomorphism of $M$ onto itself that carries $x_{1}+x_{2}+\cdots+x_{n}$ into $y_{1}+y_{2}+\cdots+y_{n}$. A nearly 1-homogeneous set is said to be nearly homogeneous. The following theorems are proved. (1) If the compact metric continuum $M$ is nearly homogeneous and is irreducible about some finite set, then $M$ is indecomposable. (2) If the bounded plane continuum $M$ is nearly homogeneous and has more than two but only a finite number of complementary domains, then $M$ is indecomposable.

(3) If $n>1$ and the bounded plane continuum $M$ is nearly $n$-homogeneous and is not a continuous curve, then $M$ is indecomposable. (4) If $n>1$ and the bounded plane continuum $M$ is $n$-homogeneous, then $M$ is a simple closed curve. (Received November $2,1953$.

\section{J. H. Case: A note on the Whitehead isomorphism theorem.}

The main theorem concerns two arcwise connected topological spaces $X$ and $Y$, a continuous map $f$ from $X$ to $Y$, and an arbitrary but fixed positive integer $M$. It is shown that if $f$ induces onto isomorphisms of the homotopy groups of $X$ and $Y$ for each dimension $n \leqq M$, then $f$ induces onto isomorphisms of the singular homology and cohomology groups of $X$ and $Y$ for each dimension $n \leqq M$ and any coefficient group. The concept of minimal subcomplex which is due to S. Eilenberg and J. A. Zilber and the concept of admissable subcomplex which is due to S. T. Hu are the main tools used in the proof. (Received November 13,1953.)

\section{1t. G. A. Dirac and Seymour Schuster: A theorem of Kuratowski.}

Theorem: A finite graph is not plane if and only if it contains a subgraph homeomorphic to the complete 5-graph or a subgraph homeomorphic to the Thomson graph (the graph whose nodes are the vertices of a hexagon and whose edges are the sides of the hexagon, plus the diagonals joining the opposite vertices). This theorem was proved by Kuratowski (Fund. Math. vol. 16, pp. 271-283). In the present paper, a much simpler proof is arrived at by induction on the number of edges of the graphs embeddable in the plane. The possible extension of the theorem to countable infinite graphs is discussed. Two definitions suggest themselves: (1) An infinite graph may be called plane if it can be embedded in the plane. (2) An infinite graph may be called plane if it can be embedded in the plane in such a way that the nodes do not have a limit point. It is shown that Kuratowski's Theorem holds if definition (1) is adopted. However, a simple counterexample is given to show that the theorem does not hold when the second definition is adopted. (Received November 14, 1953.)

\section{Samuel Eilenberg and Saunders MacLane (p): Generators for} the torsion product.

For two abelian groups $A$ and $B$ let Tor $(A, B)$ be the group with generators all symbols $\tau_{m}(a, b)$ for integers $m$ and elements $a \in A, b \in B$ with $m a=m b=0$. Subject these generators to the relations expressing linearity in each argument, and to the relations $\tau_{m n}(a, b)=\tau_{m}(a, n b)$ and $\tau_{m n}(a, b)=\tau_{m}(n a, b)$, to hold whenever both sides are defined. Then if $0 \rightarrow A \rightarrow B \rightarrow C \rightarrow 0$ is an exact sequence and $G$ any abelian group, 0 
$\rightarrow$ Tor $(A, G) \rightarrow$ Tor $(B, G) \rightarrow$ Tor $(C, G) \rightarrow A \otimes G \rightarrow B \otimes G \rightarrow C \otimes G \rightarrow 0$ is exact. In particular if $C=F / R$ is any representation of $C$ as a factor group of a free group $F$, Tor $(C, G)=$ kernel $(R \otimes G \rightarrow F \otimes G)$. This shows that the definition of Tor by generators and relations agree with the definition given in terms of $F / R$ by EilenbergCartan (Satellites des foncteurs de modules, to appear). Other properties of the torsion product, notably the Künneth relation for the homology group of torsion-free chain complexes may readily be derived from the description by generators and relations. (Received November 14, 1953.)

\section{3t. D. O. Ellis: Some saddle points in the cross-topolattice.}

Let $S$ be an infinite set and let $L$ be the topolattice of $S$ (lattice of all $T_{1}$ Kuratowski topologies on $S$, ordering by inverse inclusion of classes of open sets). Let $L^{*}$ be $L$ dually ordered and let $L^{* *}$ be the cardinal product $L \otimes L^{*}$. One shows that if $f$ is a permutation of $S$ (biuniform mapping of $S$ onto itself) and if $(a, b) \in L^{* *}$ has the property that when we take ' $a$ ' as domain topology and ' $b$ ' as range topology, $f$ is both open and continuous (homeomorphic), then $(a, b)$ is maximal in $L^{* *}$ with respect to making $f$ continuous and is minimal with respect to making $f$ open. A general discussion of lattice saddle points precedes this result. The methods are elementary. (Received October 15, 1953.)

\section{4t. G. W. Ford and G. E. Uhlenbeck: On the asymptotic number of pure and mixed Husimi trees.}

A Husimi tree is a connected graph in which no line lies on more than one cycle. A pure Husimi tree has all cycles of the same length; otherwise, it is called mixed. The number of Husimi trees has been counted by Harary and Uhlenbeck, Proc. Nat. Acad. Sci. U.S.A. vol. 39 (1953) p. 315, and by Norman (see abstract below). Using the methods of Otter, Ann. of Math. vol. 49 (1948) p. 583, the authors have determined the asymptotic numbers of pure (rooted and free) Husimi trees consisting of $n m$-gons. The form of the asymptotic behavior is the same for all $m$, but the radius of convergence of the counting series decreases with $m$ as $1 /(m-1)$. For mixed Husimi trees of arbitrary cyclic composition, the counting series in terms of number of points has the radius of convergence $0.2221 \cdots$, and the distribution of the points over the different cycles has been determined. It is a monotonic decreasing function of the cycle length. (Received November 13, 1953.)

\section{L. R. Ford, Jr.: Coset spaces and homeomorphism groups.}

Let $G$ be a homeomorphism group of $X$ and $C_{x}$ that subgroup having $x$ as a fixed point. It is shown that if $X$ is completely regular, $G$ may be made into a topological group under a uniform convergence notion. If $G / C_{x}$ is homeomorphic to $X$ one calls the $G$ topology "reasonable." Define strong local homogeneity (SLH) by: For every $x, U(x)$ there exists $V(x) \subset U(x)$ such that for every $z \in V$ there is a homeomorphism $h$ of $X$ with $h(x)=z$ and $h=I$ on $C(V)$. It is proved that a completely regular, homogeneous, SLH space has a reasonable full homeomorphism group. It is shown that locally Euclidean spaces, homogeneous 0-dimensional spaces, and normed linear spaces are SLH. In particular all manifolds are coset spaces of some topological group. (Received November 10, 1953.)

316. W. C. Fox: Critical points of real functions defined on twomanifolds. Preliminary report. 
Let $M$ be the interior of a two-manifold with boundary $J$. A real function $f$ defined on $M+J$ is Peano-interior if it is continuous, interior on $M$, and every pointinverse is locally connected in $M$. The class of Peano-interior functions on $M+J$ properly includes the pseudo-harmonic functions. Conditions on the boundary values are given which imply that $f$ may be extended to be Peano-interior on a larger manifold $M^{*}$ and to have certain canonical properties on the new boundary. If a Peano-interior function is canonical, then there is a relation between the sum $S^{*}$ of the multiplicities of the critical points of $f$ on $M^{*}$ and the first Betti number of $M^{*}$. Determination of whether or not a boundary point is a critical point is not always possible, for there is a Peano-interior function possessing two different canonical extensions for which a certain boundary point is regular under one extension and is a critical point under the other. (Received November 13, 1953.)

\section{J. S. Griffin, Jr.: A note on the cohomology theory of manifolds.}

In any topological space $X$ the Alexander-Wallace-Spanier cohomology groups may be defined using only $(p+1)$-tuples which are small relative to any given open cover $U$. But if $X$ is a differentiable manifold with Riemannian metric $g_{i j}$ and $U$ is chosen to consist exclusively of sets convex in the sense that any two points of a member $U$ of $U$ may be joined by a unique geodesic of shortest length which lies entirely within $U$, then for any $(p+1)$-tuple $\left(x_{0}, \cdots, x_{p}\right)$ which is small relative to $U$

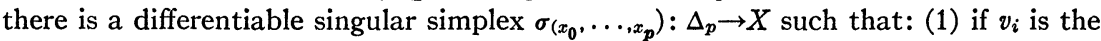
$i$ th vertex of $\Delta_{p}$, then $\left.\sigma_{\left(x_{0}\right.}, \cdots, x_{p}\right)\left(v_{i}\right)=x_{i}$, (2) if $e_{i}$ is the barycentric map of $\Delta_{p}$ onto the $i$ th face of $\Delta_{p+1}$ which preserves the order of the vertices, then $\sigma_{\left(x_{0}, \cdots, x_{p+1}\right)} e_{i}$ $=\sigma_{\left(x_{0}, \cdots, \hat{x}_{i}, \cdots x_{p+1}\right)}$. These considerations lead in a natural way to homomorphisms on the singular cochain groups and the groups of differential forms to the AlexanderWallace-Spanier cochain groups; if the manifold is taken to be compact, then using the Leray uniqueness theory these homomorphisms may be shown to induce isomorphisms on the cohomology groups. (Received September 8, 1953.)

318. Mary-Elizabeth Hamstrom: A characterization of plane continuous curves which are filled up by continuous collections of continuous curves.

Let $M$ be a compact plane continuous curve. R. D. Anderson has shown (Continuous collections of continuous curves in the plane, Proc. Amer. Math. Soc. vol. 3 (1952) pp. 647-657) that if $M$ is the sum of the elements of a continuous collection of simple closed curves, then $M$ is an annulus. The author has shown (Proc. Amer. Math. Soc. vol. 4 (1953) pp. 240-243) that if $M$ is the sum of the elements of a continuous collection of arcs which is an arc with respect to its elements, then $M$ is a closed disc. The principal theorem of the present paper establishes that $M$ is the sum of the elements of a continuous collection of nondegenerate continuous curves if and only if every boundary of a complementary domain of $M$ is a simple closed curve and the collection of these boundaries satisfies certain conditions, which are too lengthy to be presented here. (Received November 5, 1953.)

\section{Melvin Henriksen: $A$ generalization of a theorem of Hewitt.}

Let $C(X, R)$ denote the ring of all real-valued continuous functions on a completely regular space $X$, and let $M$ be any maximal ideal of $C(X, R)$; then the residue class field $C(X, R) / M$ is an ordered field containing the real field $R$ (Hewitt, Trans. Amer. Math. Soc. vol. 64 (1948) pp. 45-99, Theorem 41). $C(X, R) / M$ is called a hyper-real field if it contains $R$ properly. Theorem: If $X$ is any completely regular 
space, and if $C(X, R) / M$ is hyper-real, then $C(X, R) / M$ contains no countable cofinal subset. This generalizes a theorem of Hewitt, who had obtained the result under the additional restriction that $X$ is both locally compact and $\sigma$-compact (loc. cit., Theorem 43). (Received November 14, 1953.)

320. F. E. P. Hirzebruch: A generalization of Todd's arithmetic genus. Preliminary report.

For nonsingular algebraic varieties $V_{n}$ the integer $\chi^{p}\left(V_{n}\right)$ is defined by $\sum_{i=0}^{n}(-1)^{i} h^{p, i}$. Here $h^{p, q}$ denotes the dimension of the linear space of harmonic forms of type $(p, q)$ on $V_{n}$. It is conjectured (Proc. Nat. Acad. Sci. U.S.A. vol. 39 (1953) pp. 951-956) that $\chi^{0}\left(V_{n}\right)$ equals the Todd genus of $V_{n}$ which is a polynomial $T_{n}\left(c_{1}, c_{2}, \cdots, c_{n}\right)$ of weight $n$ in the Chern classes $c_{i}$, where $c_{i} \in H^{2 i}\left(V_{n}, Z\right)$. In this paper polynomials $T_{n, p}\left(c_{1}, c_{2}, \cdots, c_{n}\right)$ are introduced and it is conjectured that $T_{n, p}\left(c_{1}, c_{2}, \cdots, c_{n}\right)=\chi^{p}\left(V_{n}\right)$. Using a result of Kodaira-Spencer, this equation is proved for every complete intersection $W_{n}$ of hypersurfaces in some projective space. It provides a method for calculating all the $h^{p, q}$ of $W_{n}$. (Lefschetz's theorem implies that $h^{p, q}=\delta_{q}^{p}$ for $p+q \neq n$.) The polynomials $T_{n, p}$ make sense for a compact almostcomplex manifold $M_{n}$. It is proved that $2^{n-1} T_{n, p}$ is an integer for every $M_{n}$. This was proved earlier for $p=0$ (loc. cit.). For $p=1$ one gets the corollary: If in an almostcomplex manifold $M_{n}$ the Chern classes $c_{i}$ vanish for $i \leqq[n / 2]$, then $2^{n-2} c_{n}$ is divisible by $(n-1)$ !. This implies the theorem of Borel-Serre that the spheres $S^{2 n}$ are not almost-complex for $n \geqq 4$. Remark: $\sum_{p=0}^{n}(-1)^{p} \chi^{p}\left(V_{n}\right)$ is the Euler-Poincare characteristic of $V_{n}$ and $\sum_{p=0}^{n} \chi^{p}\left(V_{n}\right)$ is the index $\tau\left(V_{n}\right)$ (Hodge). The polynomials $T_{n, p}$ have the corresponding properties. (Received November 12,1953.)

321. F. B. Jones: On a property related to separability in metric spaces.

An example is given of a metric space in which the following proposition is false: If no separable subset of a metric space $S$ is uncountable, then $S$ is the sum of countably many discrete sets. The example possesses other properties of a related nature. (Received November 14, 1953.)

\section{J. W. Keesee: A separation theorem for manifolds.}

Let $(X, A)$ be a compact topological pair where $X$ is a connected $(n+1)$-dimensional Euclidean manifold. Let $G$ be the additive group of integers if $X$ is orientable, otherwise let $G$ be cyclic of order two. The inclusion map $i: A \rightarrow X$ induces a homomorphism $i^{*}: H^{n}(X) \rightarrow H^{n}(A)$ where $H^{n}(X)$ denotes the $n$-dimensional Cech cohomology group of $X$ with coefficients in $G$. Let $N$ be the number (finite or countable) of components of $X-A$. Let $\sum_{j-1}^{N-1} G_{j}$ represent the weak sum of $N-1$ groups each isomorphic to $G$. Theorem: The quotient group $H^{n}(A) / i^{*}\left(H^{n}(X)\right)$ is isomorphic to $\sum_{j=1}^{N-1} G_{j}$. (Received November 12, 1953.)

323t. A. B. Lehman: On pseudo-linear quadruples in a metric lattice. Preliminary report.

Four points in a metric space form a pseudo-linear quadruple if and only if the determinant of their distances vanishes. A necessary and sufficient condition that four elements of a metric lattice form a pseudo-linear quadruple, $Q(a, b ; c, d)$, is that (1) $a \wedge b=c \wedge d$ and $a \bigvee b=c \bigvee d$, and (2) $a, b, c, d$ distribute among themselves. The sublattice generated by a pseudo-linear quadruple is isomorphic with $2^{2}, 2^{3}$, or $2^{4}$ 
depending on the comparability of the elements concerned. The transitivity: $Q(a, c ; b, d)$ and $Q(c, e ; d, f)$ imply $Q(a, e ; b, f)$ is a criterion for distributivity in metric lattices. (2) implies (1) if and only if the lattice has no four-element chain. Lattices in which (1) implies (2) are called quasi-distributive. Any sublattice generated by three elements is quasi-distributive; accordingly, quasi-distributivity is determined by sublattices generated by four elements. Numerous "forbidden pictures" for quasidistributive lattices exist, the simplest being the multiple projective root. Characterization and classification of these is possible. Generation of sets metrically approximating Condition (1) is considered. (Received November 24, 1953.)

\section{P. S. Mostert: Sections in principal fibre spaces.}

Let $B=\{X, B, p, G\}$ be a principal fibre space (Serre, C. R. Acad. Sci. Paris vol. 229 (1949) pp. 1295-1297) such that $X, B$, and $G$ are inverse limits of $\left\{X_{\alpha} ; \pi_{\alpha}^{B}\right\}$, $\left\{B_{\alpha} ; \bar{\pi}_{\alpha}^{\beta}\right\},\left\{G_{\alpha} ; \rho_{\alpha}^{\beta}\right\}$, respectively, $\alpha, \beta \in \Lambda, \alpha<\beta$, such that $\mathcal{B}_{\alpha}=\left\{X_{\alpha}, B_{\alpha}, p_{\alpha}, G_{\alpha}\right\}$ is a principal fibre space, and $p_{\alpha} \pi_{\alpha}^{\beta}=\bar{\pi}_{\alpha}^{\beta} p_{\beta}, \pi_{\alpha}^{\beta}\left(g_{\beta} x_{\beta}\right)=\rho_{\alpha}^{\beta}\left(g_{\beta}\right) \pi_{\alpha}^{\beta}\left(x_{\beta}\right)$, where all mappings are open and continuous. We shall say that $\mathscr{B}$ is the inverse limit of $\left\{\mathcal{B}_{\alpha} ; \Lambda\right\}$. The system $\left\{B_{\alpha} ; \Lambda\right\}$ is said to satisfy the property (P) if (i) $\Lambda$ has the finite chain property (i.e., any chain between $\alpha$ and $\beta$ has at most a finite number of elements and $\Lambda$ has a least element 1), and (ii) there exists an open set $U=\boldsymbol{P}_{\alpha} U_{\alpha} \cap B$, where $U_{\alpha}=\left(\pi_{1}^{\alpha}\right)^{-1}\left(U_{1}\right)$, $U_{1}$ an open set in $B_{1}$ such that, for a fixed point $x=\left\{x_{\alpha}\right\} \in X$, the sets $F_{\alpha}$ of cross sections of $U_{\alpha}$ mapping a point of $U_{\alpha}$ into $x_{\alpha}$ have the property that, if $\alpha<\beta$ and $f_{\alpha}$ $\in F_{\alpha}$, then there exists $f_{\beta} \in F_{\beta}$ such that $\pi_{\alpha}^{\beta} f_{\beta}=f_{\alpha} \bar{\pi}_{\alpha}^{\beta}$ on $U_{\beta}$, and conversely; moreover $F_{1}$ is nonempty. The following then holds: (1) If $\mathcal{B}$ is an inverse limit of $\left\{\mathcal{B}_{\alpha} ; \Lambda\right\}$ satisfying $(\mathrm{P})$, then there is a cross section defined on $U$. This then gives: (2) If $X$ is a locally compact group, $G$ a closed subgroup such that $B=X / G$ is finite-dimensional, then $G$ has a local cross section; (3) If $\mathcal{B}$ is a principal fibre space with locally compact finite-dimensional structural group, and $B$ is locally arcwise connected and locally simply connected, then local cross sections exist for $\mathcal{B}$. Other related results are obtained. (Received November 9, 1953.)

\section{R. Z. Norman: On the number of linear graphs with given blocks.}

The powerful methods of Poblya are employed to solve some problems of counting various kinds of graphs. These results are used by Ford and Uhlenbeck (see abstract above). A pure Husimi tree is a connected graph in which every line lies in exactly one cycle of length $n$. A generating function is developed for counting pure Husimi trees using the known function for rooted pure Husimi trees (Harary and Uhlenbeck, Proc. Nat. Acad. Sci. U.S.A. vol. 39 (1953) p. 315). This result is extended to count arbitrary Husimi trees. A block of a graph is defined as a maximal connected subgraph with no articulation points. A connected graph can, of course, itself be a block. Let $B$ be a collection of blocks. Rooted connected graphs whose blocks are isomorphic to blocks of $B$ are counted, and from this the counting of analogous unrooted graphs is accomplished, using the group of the block and a certain subgroup derived from it. Similar results are obtained for directed graphs, for graphs of strength $s$ (see Harary, Bull. Amer. Math. Soc. Abstract 58-6-717), and for simplicial complexes. The restriction to connected graphs is easily eliminated. (Received November 13,1953.)

326t. Joel Pitcairn: A discrete analogue of the Weierstrass-Stone theorem.

The following analogue of the Weierstrass-Stone theorem is proved: Let $F$ be a field with discrete topology and let $H$ be a set of continuous functions from a compact 
Hausdorff space $X$ to $F$ which separates the points of $X$. Then every continuous function from $X$ to $F$ is a polynomial with respect to $H$, with coefficients in $F$. A number of applications are given, among which is the following: An algebra over $G F\left(p^{n}\right)$ is called a $p^{n}$-algebra in case every $x$ in it satisfies $x^{q}=x$, where $q=p^{n}$. Let $\alpha$ be an infinite cardinal. Then there exists a unique compact boolean space $X$ and a point $z$ in $X$ such that for every $p, n$, the free $p^{n}$-algebra with $\alpha$ generators is the algebra of all continuous functions from $X$ to $G L\left(p^{n}\right)$ which vanish at $z$. If the phrase "which vanish at $z$ " is omitted one gets the free $p^{n}$-algebra with unit with $\alpha$ generators. (Received October 19, 1953.)

\section{7t. Joel Pitcairn: The existence of Banach limits.}

A bounded sequence $x=\left(x_{n} \mid n \in N\right)$ is a function from the set $N$ of positive integers into a compact subset of the reals $R$. Let $X$ be the algebra over $R$ of all bounded sequences. Let $A$ be an ultrafilter on $N$ containing the finite-complement filter. Clearly the functional $\Lambda=(\lim (\mathcal{A}) x \mid x \in X)$ is a homomorphism of $X$ onto $R$, and liminf $x$ $\leqq x \Lambda \leqq \limsup x(x \in X)$. Define $x \Gamma=\left(\left(\sum_{i=1}^{n} x_{i}\right) / n \mid n \in N\right) \quad(x \in X)$. Clearly $\Gamma$ is a linear transformation of $X$ into itself, and the functional $B=\Gamma \Lambda$ is linear and has the property that liminf $x \leqq x B \leqq \limsup x(x \in X)$. Let $x=\left(x_{n} \mid n \in N\right)$ and $y=\left(x_{n+1} \mid n \in N\right)$. Then $(y-x) \Gamma=\left(\left(x_{n+1}-x_{1}\right) / n \mid n \in N\right)$, and so $0=\lim (y-x) \Gamma=(y-x) \Gamma \Lambda=y B-x B$. Thus $B$ is a Banach limit. (Received October 19, 1953.)

\section{8t. R. L. Plunkett: Some implications of semi-1-connectedness.}

A topological space is semi- $n$-connected at $x \in X$ provided there exists an open set $U$ containing $x$ such that every Čech $n$-cycle on a compact subset of $U$ bounds on $X$. The following theorems are proved, where $X$ is a separable metric continuum: (1) If $X$ is semi-1-connected at $x \in X$, then there exists an open set $V$ containing $x$ such that, if $Q$ is a region with $\bar{Q} \subset V$, then no continuum in $V-Q$ intersects two components of $\bar{Q}-Q$. (2) If $X$ is locally connected and is semi-1-connected at a nonlocal separating point $x$, then $X$ is locally peripherally connected at $x$. (3) If $X$ is locally connected and is semi-1-connected at a nonlocal separating point $x$, then there exist arbitrarily small open sets $V$ containing $x$ such that, if $Q$ is any region contained in $V$, then there exists a component $C$ of $\bar{Q}-Q$ such that the component of $X-C$ containing $Q$ is contained in $V$. (4) If $T$ is a compact totally disconnected set in a locally connected $X$ such that no point of $T$ is a local separating point, if $X$ is semi-1connected at each $x \in T$, and if $d$ is a positive number, then there exists a finite open covering $\left\{Y_{1}, Y_{2}, \cdots, Y_{k}\right\}$ of $T$ such that (1) diam $\left(Y_{i}\right)<d$, for each $i$, (2) $\bar{Y}_{i}-Y_{i}$ is connected, for each $i$, and (3) $Y_{i} \cdot Y_{j}=0$, if $i \neq j$. (Received November 13,1953.)

\section{9t. B. A. Rattray: An antipodal point-orthogonal point theorem.}

It is shown that, if $T$ is any continuous mapping of the metric $n$-sphere into itself which carries antipodal points into antipodal points, then there is some set of $(n+1)$ mutually orthogonal points whose images under $T$ are also mutually orthogonal. The main tool used in the proof is the theory of intersection numbers. (Received November 16, 1953.)

\section{J. C. Rogers: Cross sections in fibre bundles.}

Let $B$ be a fibre bundle with base space $K$, a finite cellular complex, and connected fibre $Y$, which need not have a vanishing fundamental group nor be simple in any dimension. Then, necessary and sufficient conditions are given for the extension of a cross section (defined on a subcomplex, $L$ ) over $K^{2} \cup L$. Homotopy on $K^{1} \cup L$ divides 
cross sections into homotopy equivalence classes which are in 1-1 correspondence with the "admissible" homomorphisms, $\phi: \pi_{1}\left(K, x_{0}\right) \rightarrow \pi_{1}\left(B, b_{0}\right), B$ being the bundle space. Higher dimension deformation and extension theorems are developed, including the "one stage" homotopy classification theorem: for any $q \geqq 2$, the homotopy equivalence classes of cross sections defined on $K^{q} \cup L$, extendable over $K^{q+1} \cup L$, and homotopic on $K^{q-1} \cup L$ are in 1-1 correspondence with a certain factor group of $H^{q}\left(K, L ; \mathfrak{B}^{q}(f)\right)$, where $\mathscr{B}^{q}(f)$ is a suitably defined coefficient bundle. Applications are given, including the complete classification of the fields of real nonoriented line elements over $P^{n}$, projective $n$-space, $n>2$. (Received November 3,1953 .)

\section{1t. J. P. Roth: An investigation in Morse theory.}

An axiomatization of Morse critical point theory is given in terms of a triple $(S, f, H)$ consisting of a Hausdorff space $S$, an arbitrary real-valued mapping $f$ of $S$, and an arbitrary homology theory $H$. The principal tools are the notion of filter and the Eilenberg-Steenrod formulation of homology theory. This approach generalizes previous presentations and serves to clarify the relations between various levels of and approaches to Morse theory. The Morse relations, in the form of group isomorphisms, are derived without any finiteness assumptions (e.g. finite number of critical points). Let $S_{c}=\{x \mid f(x) \leqq c\}$; if $S_{c}$ is compact for each $c, H$ is a homology theory continuous "on $S$ and $f$ " and $S$ is $f$-reducible, then all the axioms are satisfied so that the Morse relations hold, and there exists a critical point at each critical value. Applications are made to minimal surface theory and the theory of optimum guided missile trajectories. (Received November 18, 1953.)

\section{Mary E. Rudin: Connected subsets of a plane set.}

In a paper entitled Some remarks on connected sets (Bull. Amer. Math. Soc. vol. 50 (1944) p. 445) Erdös raises the question of the existence of a nondegenerate connected set which is topologically equivalent to all of its nondegenerate connected subsets. In this paper it is shown that such a set exists in the plane if the hypothesis of the continuum is true. (Received November 10, 1953.)

\section{Nobuo Shimada and Hiroshi Uehara (p): Some remarks on} Adem extension theorem.

This paper is concerned with the classification of mappings of an $(n+2)$-dimensional complex into an $(n-1)$-connected space $(n>2)$ by using generalized Adem's operation, $q^{i}$-operation introduced elsewhere by the authors, and Steenrod's squaring operation. (Received November 13, 1953.)

\section{G. L. Thompson: On lattices of linear equations.}

Let $k$ be a sfield and $V_{n}$ the $n$-dimensional (left) vector space over $k$. Let $N$ be the $n$-dimensional (modular) lattice of all subspaces of $V_{n}$ and let $L$ be an $l$-dimensional sublattice of $N$ which has the same all and zero element as $N$ does. It is shown that a basis can be chosen in $V_{n}$ such that to every subspace $A$ in $L$ there corresponds an $l \times l$ matrix $\sum(A)$ where the matrix $\sum(A)$ is in row Hermite normal form and its entries are matrices of suitable dimensions with entries from $k$. The number of nonzero entries on the main diagonal of $\sum(A)$ is the dimension of $A$ measured in $L$, and the rows of $\sum(A)$ form a basis for vectors in $A$. By means of the matrix $H(A, B)$ $=\sum(A)\left[I-\sum(B)\right]$ the matrices $\sum(A \cup B)$ and $\sum(A \cap B)$ corresponding to the union and intersection of two elements $A$ and $B$ of $L$ can be found. If $A$ and $B$ are 
elements of $L$ such that $A / A \cap B$ and $B / A \cap B$ are projective in $L$, and $Q_{a}(A, B)$ is a space projectively related to $L$ (c.f. R. M. Thrall, Canadian Journal of Mathematics vol. 4 (1952) p. 229) the problem of finding $\sum\left(Q_{a}(A, B)\right)$ is solved. (Received November 16, 1953.)

335. R. F. Williams: Reductions of open mappings.

Suppose $f$ is a continuous open mapping of a compact metric space $X$ onto a topological space $Y$. The following three theorems will serve to illustrate the three types of results obtained: (1) if $U$ is a nonempty open subset of $X$ such that $f^{-1}(y)$ is locally connected for every $y \in f(U)$, then there exists a nonempty open subset $V$ of $X$ such that (a) $\bar{V} C U$, (b) for each $y \in f(V), f^{-1}(y) \cdot V$ lies in a single component of $f^{-1}(y) \cdot \bar{V}$, and (c) if $y \in f(\bar{V})$ then there exists a point $x \in f^{-1}(y)$ and a neighborhood $N$ of $x$ with $f^{-1} f(V) \cdot N \subset V$; (2) if for each $y \in Y, f^{-1}(y)$ is a decomposable continuum, then there exists a proper closed subset $X^{\prime}$ of $X$ such that $f \mid X^{\prime}$ is monotone and onto $Y$; and (3) if $Y$ is perfect and $f^{-1}(y)$ is connected and locally connected for each $y \in Y$, then there exists a closed subset $X^{\prime}$ of $X$ such that $f \mid X^{\prime}$ is monotone and onto $Y$ and such that no closed proper subset of $X^{\prime}$ maps onto $Y$. (Received November 6,1953.)

L. W. Cohen, Associate Secretary 